\title{
Predictors of Posttraumatic Growth in Stroke Survivors
}

\author{
Grace Kelly
}

Supervised by:

Professor Reg Morris

May 2015

Thesis submitted in partial fulfilment of the requirement for the degree of Doctor of Clinical Psychology at Cardiff University and the South Wales Doctoral Programme in Clinical Psychology 


\section{DECLARATION}

This work has not been submitted in substance for any other degree or award at this or any other university or place of learning, nor is being submitted concurrently in candidature for any degree or other award.

Signed (candidate)

Date

\section{STATEMENT 1}

This thesis is being submitted in partial fulfillment of the requirements for the degree of D.Clin.Psy.

Signed (candidate)

Date

\section{STATEMENT 2}

This thesis is the result of my own independent work/investigation, except where otherwise stated.

Other sources are acknowledged by explicit references. The views expressed are my own.

Signed (candidate)

Date

\section{STATEMENT 3}

I hereby give consent for my thesis, if accepted, to be available for photocopying and for interlibrary loan, and for the title and summary to be made available to outside organisations.

Signed (candidate)

Date

\section{STATEMENT 4: PREVIOUSLY APPROVED BAR ON ACCESS}

I hereby give consent for my thesis, if accepted, to be available for photocopying and for interlibrary loans after expiry of a bar on access previously approved by the Academic Standards \& Quality Committee.

Signed (candidate)

Date 


\section{Acknowledgements}

I would like to thank all the stroke survivors who so generously gave their time to participate in this study. Without your participation, this research would not have been possible. It was a privilege to be able hear about your experiences of life after stroke. I was particularly struck by the stories of resilience and I felt inspired by your candour and openness to new experiences.

I am truly grateful to my supervisor, Reg, who has provided me with support and guidance throughout this research journey. I really appreciate how you always made time to support this research from attending an ethics committee meeting with me, to checking last minute drafts. Your enthusiasm for research and innovation has been really inspiring. I would also like thank to my appraisal tutor, Prof. Neil Frude, for all your support over the past three years.

A special thanks goes to Dr. Hamsaraj Shetty and Alyson Clemow for supporting this research and identifying people to take part in the study.

Thank you class of 2012. I feel so grateful that I have been able to share my journey on the DClinPsy with such a lovely bunch of people. It's been a blast!

A huge 'go raibh míle maith agaibh' goes to my family in Ireland who have been a tremendous support throughout this whole process and life in general: Mum, Dad, Edward and Emma. Last, but certainly not least, I would like to thank my partner, Mo, for his patience and support throughout. I love how you always find a way to make me smile. I'm looking forward to being able spend more time with you and to making memories! One last thank you goes to Bob and Bertie for always giving me a cuddle when I felt stressed. 


\section{Abstract}

Aims: Posttraumatic growth (PTG) describes the positive changes that can be experienced following exposure to a traumatic event such as stroke. There are no known studies examining the development of PTG in stroke survivors over time. This study sought to address this gap in the literature by investigating the predictors of PTG in stroke survivors over time.

Design: A longitudinal survey design was employed to examine the relationship between PTG and a range of predictor variables at two time points within a year post-stroke.

Method: Forty-three stroke survivors were recruited through a stroke outpatient clinic in the National Health Service. At both time points all participants completed the Posttraumatic Growth Inventory (PTGI), Rumination Scale, Impact of events scale (IES-R), Multidimensional Scale of Social Support (MSPSS), the Barthel Index and the COPE scale.

Results: This study found that participants experienced PTG in the acute phase post-stroke (i.e. time 1) but experienced significantly more growth six months later (i.e. time 2). Reported levels of PTG were similar to comparable literature. A stepwise linear regression revealed that active coping at time 1, rumination at time 1 and age accounted for $48 \%$ of the variance in PTG at time 2. Active coping at time 1 was associated with PTG at time 2 but it did not mediate the effect of social support on PTG. However, this study found that rumination mediated the relationship between posttraumatic stress symptoms and PTG over time.

Conclusions: This study suggests that PTG can develop in stroke survivors over time and supports the theory that deliberate rumination promotes PTG (Tedeschi \& Calhoun, 1995, 2004). Notably, denial coping at time 1 was associated with rumination at time 2 . Such findings reflect the complexity of post-stroke psychological adjustment. Theoretical implications of the study findings are discussed and areas for future research considered. 


\section{Contents}

1. Introduction

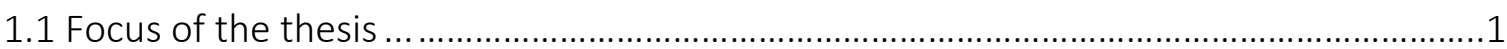

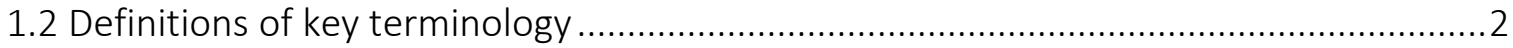

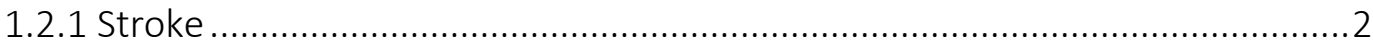

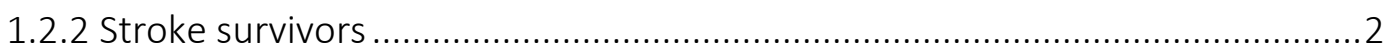

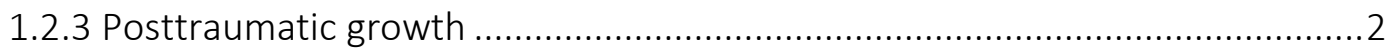

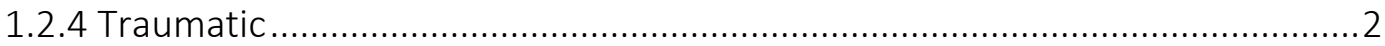

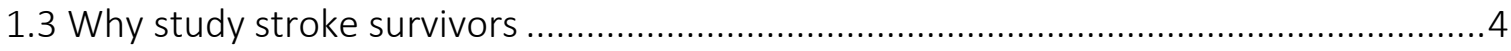

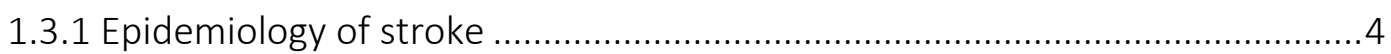

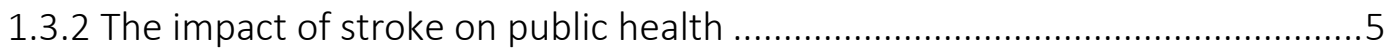

1.3.3 The impact of stroke at an individual level ................................................

1.3.4 Psychological adjustment following stroke....................................................

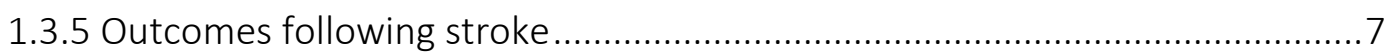

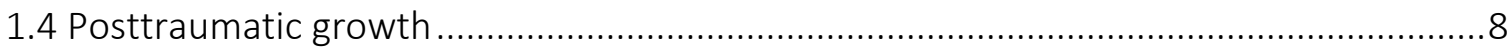

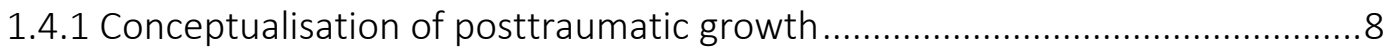

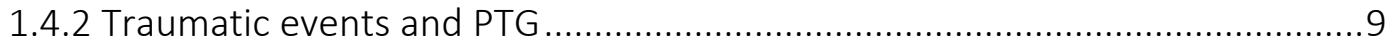

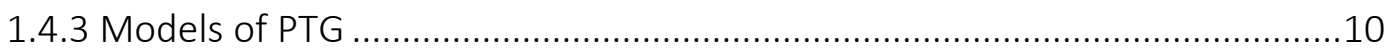

1.4.3.1 Model of life crises and personal growth (Schaefer \& Moos, 1992) .......10

1.4.3.2 Revised model of PTG (Tedeschi \& Calhoun, 1995, 2004) ......................11

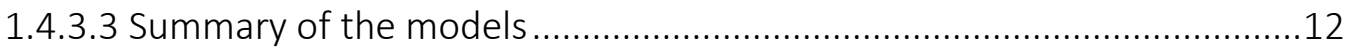

1.4.3.4 Empirical support for models in the literature .....................................14

1.4.4 Factors associated with PTG in the literature ................................................15

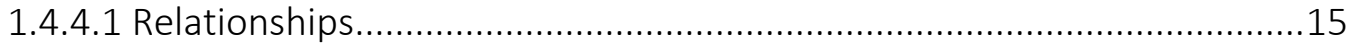

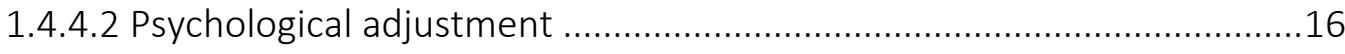

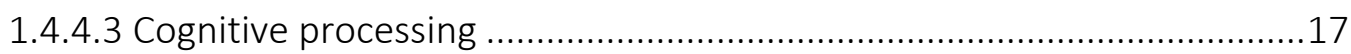

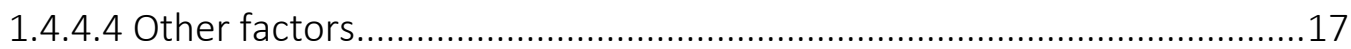

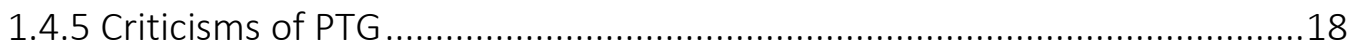




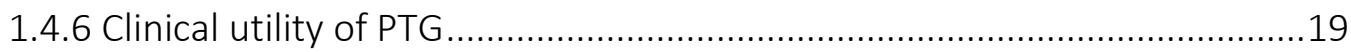

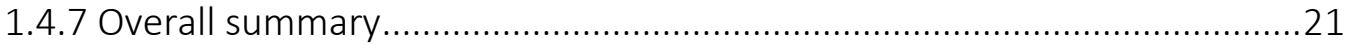

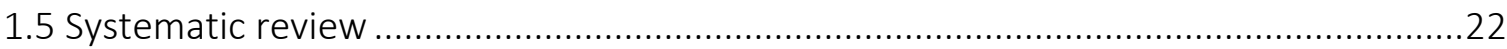

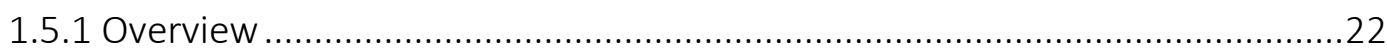

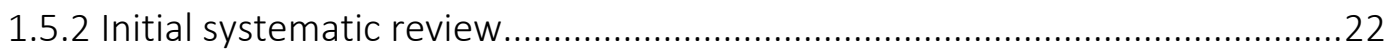

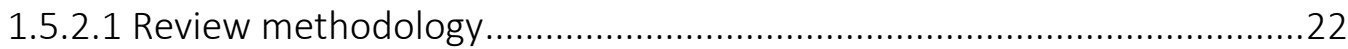

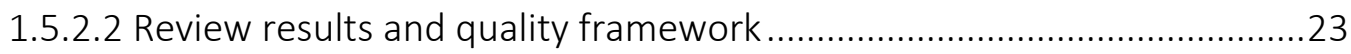

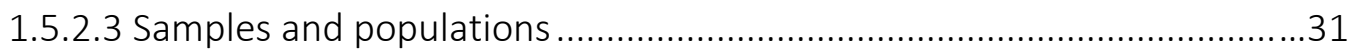

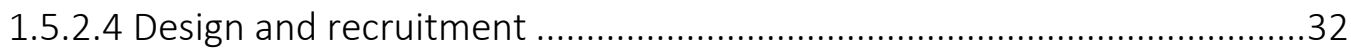

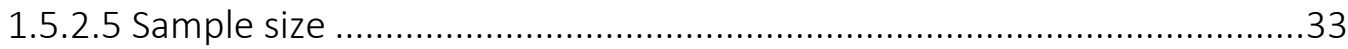

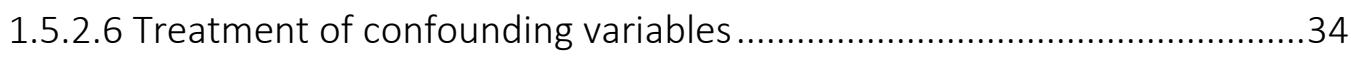

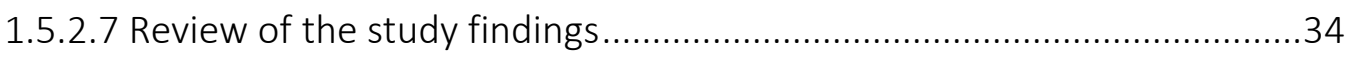

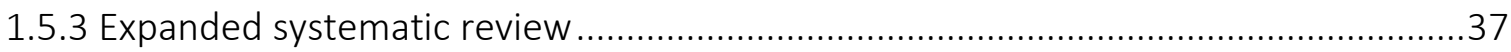

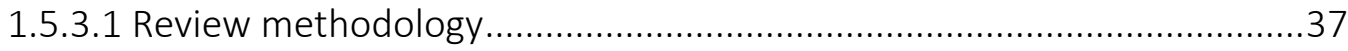

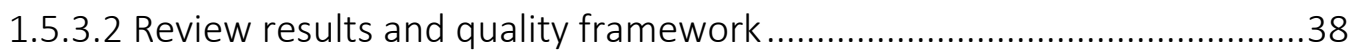

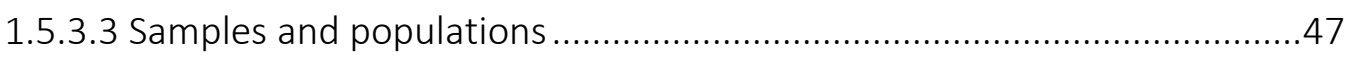

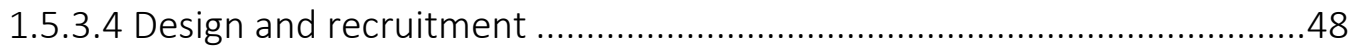

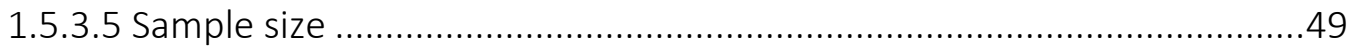

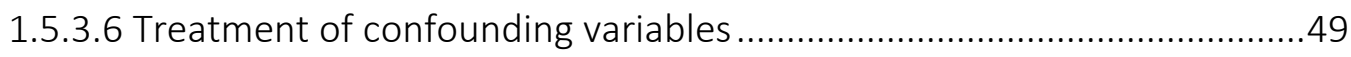

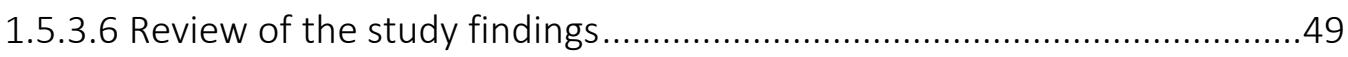

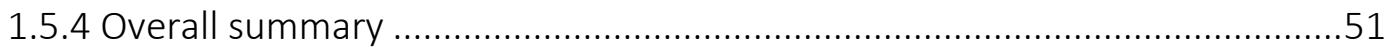

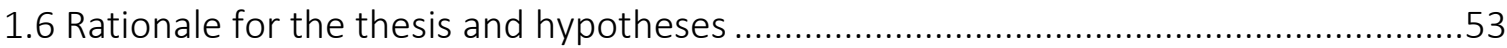

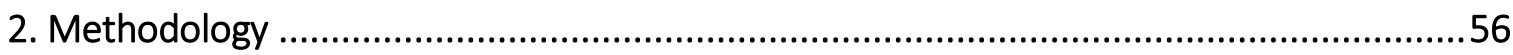

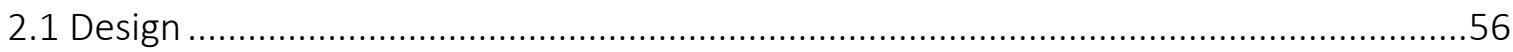

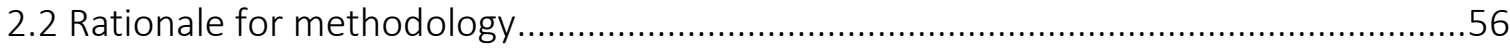

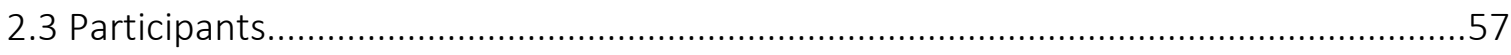

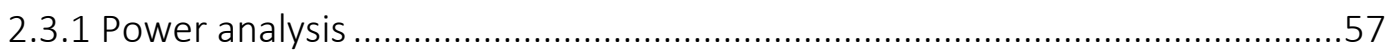




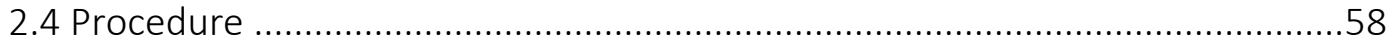

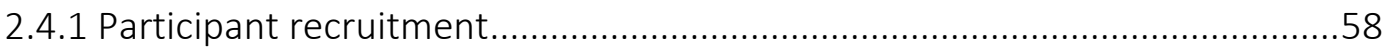

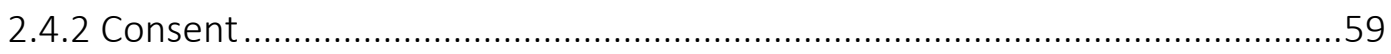

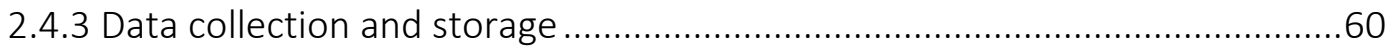

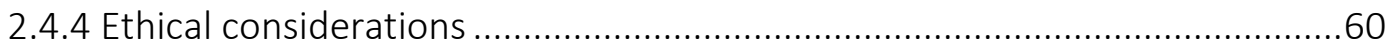

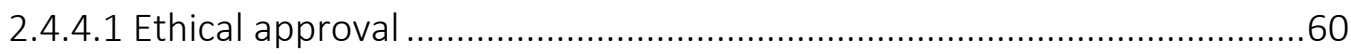

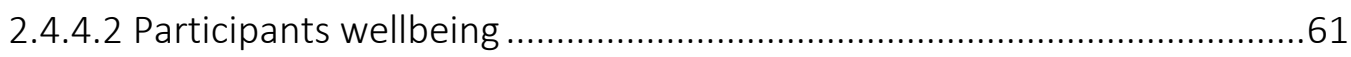

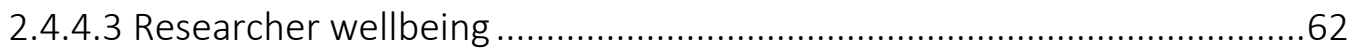

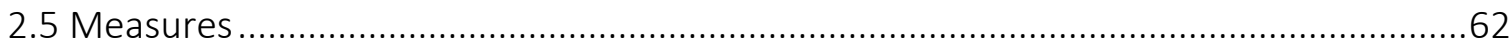

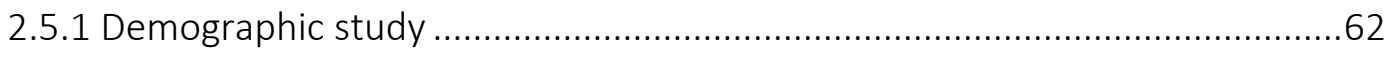

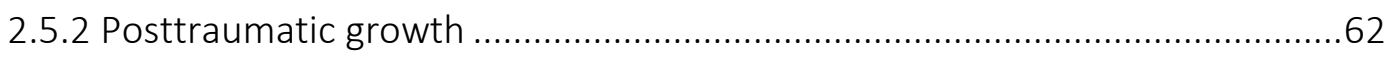

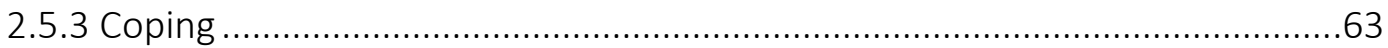

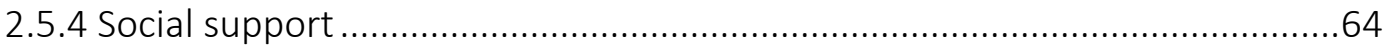

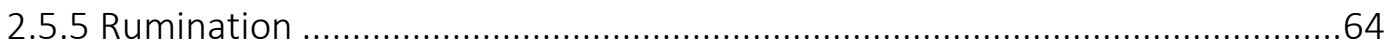

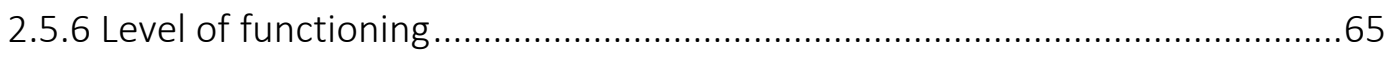

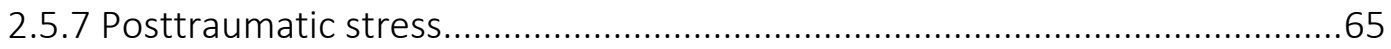

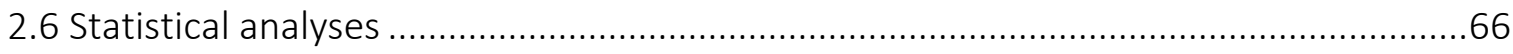

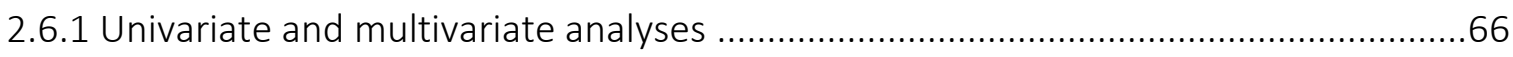

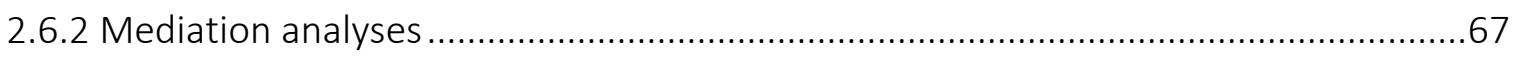

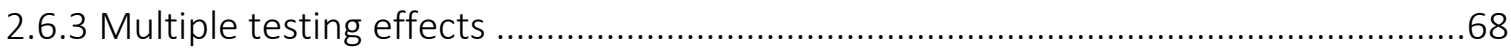

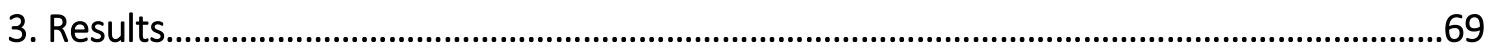

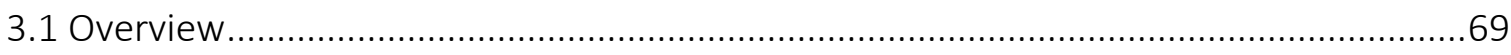

3.2 Data cleaning and assumptions for parametric testing .............................................69

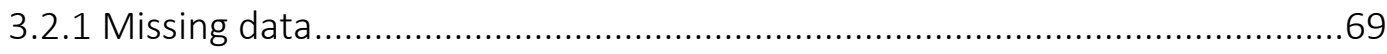

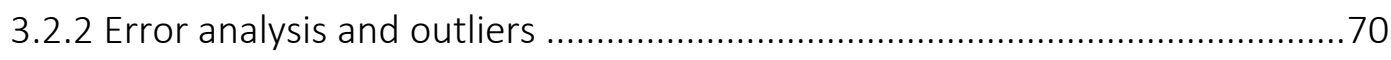

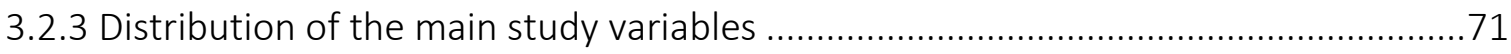


3.2.5 Approach to planned statistical analysis .................................................. 72

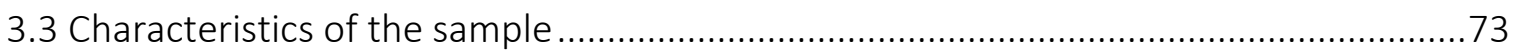

3.3.1 Demographic and background characteristics of the sample .......................73

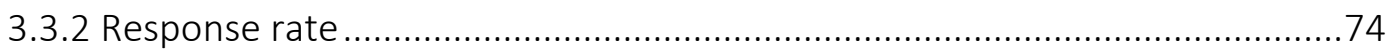

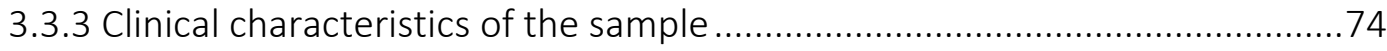

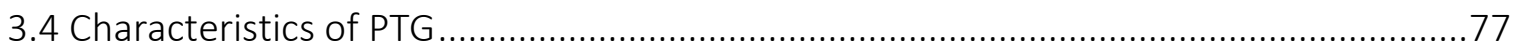

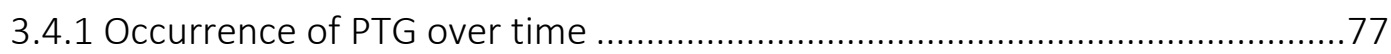

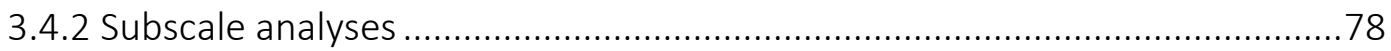

3.4.3 Comparison with previously reported data ...............................................

3.5 Social support, coping, daily functioning and posttraumatic growth ..........................79

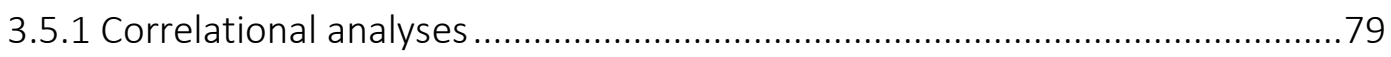

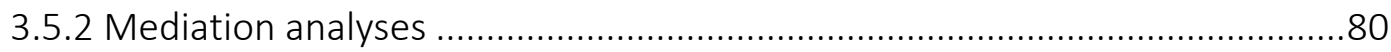

3.6 Rumination, coping, social support, posttraumatic stress and PTG ...........................81

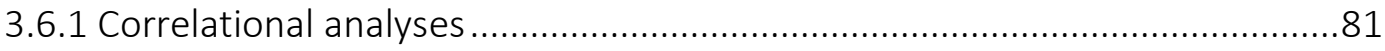

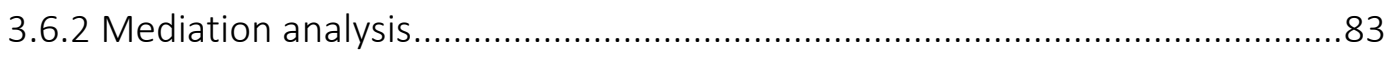

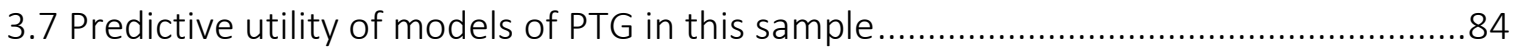

3.7.1 Tedeschi and Calhoun (2004) model of PTG and this sample .......................84

3.7.2 Schaefer and Moos (1998) model of PTG and this sample ..........................87

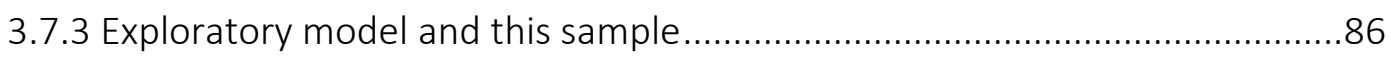

3.7.4 Summary of findings from regression analyses .........................................8

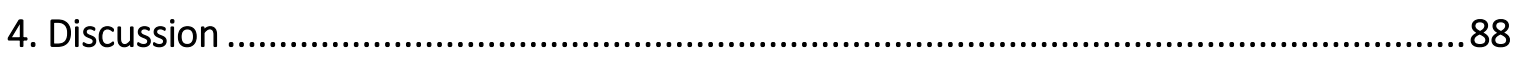

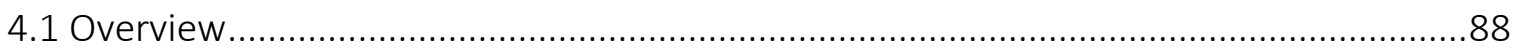

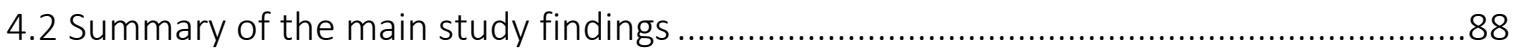

4.3 Main study findings and their relation to past research.............................................

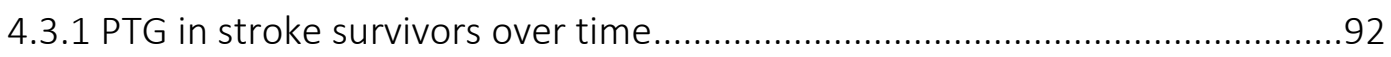

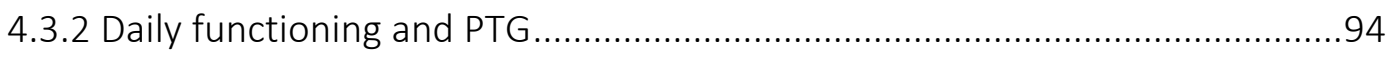


4.3.3 Social support and PTG

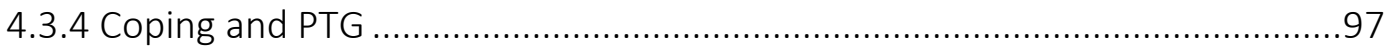

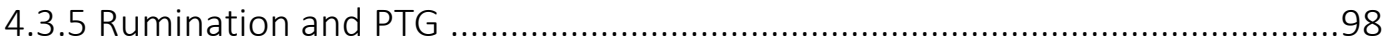

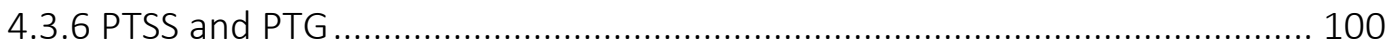

4.3.7 Social support as a mediating variable .......................................................... 102

4.3.8 Rumination as a mediating variable ...................................................... 103

4.3.9 Predictive model of PTG in this sample .................................................. 103

4.4 Summary of the theoretical implications of the current findings .............................. 104

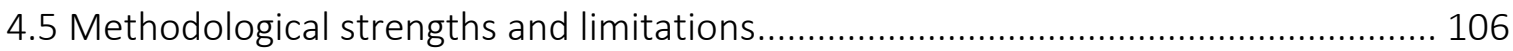

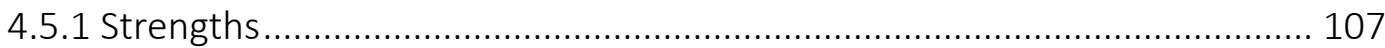

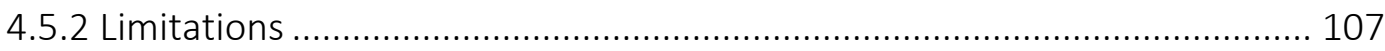

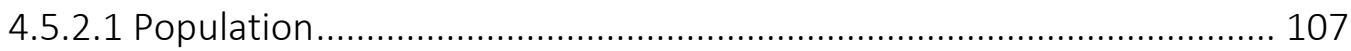

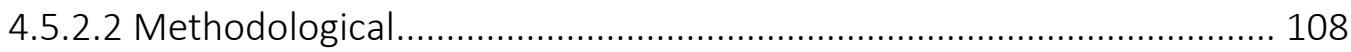

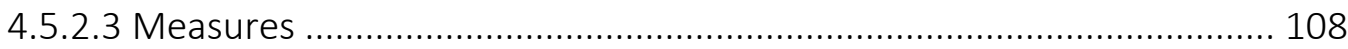

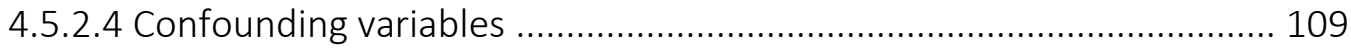

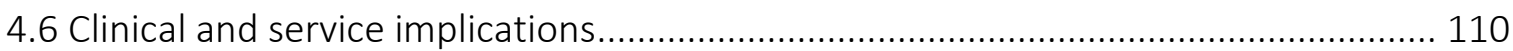

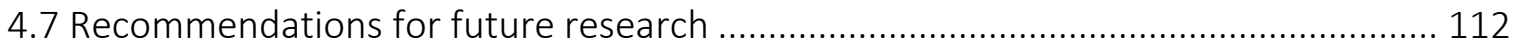

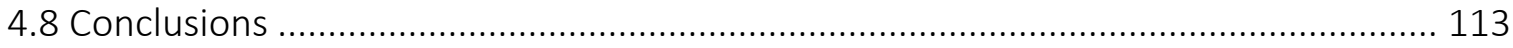

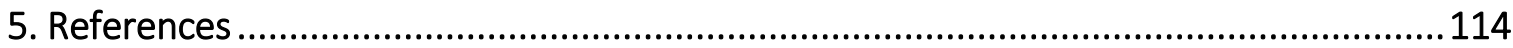




\section{List of Tables}

\section{Chapter One}

Table 1.1 Summary of the studies which met the inclusion criteria for the initial systematic review.

Table 1.2 Evaluation of correlational/cross sectional studies using the SURE Quality Framework for the initial review.

Table 1.3 Evaluation of the qualitative study using the SURE Quality framework for the initial review.

Table 1.4 Summary of the studies which met the inclusion criteria for the expanded systematic review.

Table 1.5 Evaluation of correlational/cross sectional studies using the SURE Quality Framework for the expanded review.

Table 1.6 Evaluation of qualitative a study using the SURE Quality Framework for the expanded review.

\section{Chapter Three}

Table 3.1 Socio-demographic and background characteristics of the sample.

Table 3.2 Descriptive statistics for the key variables over time.

Table 3.3 Bivariate correlations between the main study variables.

Table 3.4 Comparison of PTGI in the current study with previously reported data.

Table 3.5 Summary of key findings relating to coping, social support, functioning and PTG.

Table 3.6 Simple mediation model of the indirect effects of social support on PTG through active coping and acceptance coping.

Table 3.7 Summary of key findings relating to rumination, coping, PTSS and PTG. 
Table 3.8 Simple mediation model of the indirect effects of PTSS on PTG at time 2 through rumination

Table 3.9 Simple mediation model of the indirect effects of rumination on PTG through PTSS and acceptance coping.

Table 3.10 Simple mediation model of the indirect effects of rumination on PTG through active coping.

Table 3.11 Summary of hierarchical regression for Tedeschi and Calhoun (2004) model of PTG.

Table 3.12 Summary of hierarchical regression for Tedeschi and Calhoun (2004) model of PTG.

Table 3.13 Summary of stepwise regression findings using all variables as predictors of PTGI at time 2 . 


\section{List of Figures}

\section{Chapter One}

Figure 1.1 Model of life crises and personal growth (Schaefer \& Moos, 1992).

Figure 1.2 Revised model of PTG (Tedeschi \& Calhoun, 1995, 2004).

Figure 1.3 Flow chart of the procedure for selecting studies for initial systematic review.

Figure 1.4 Flow chart of the procedure for selecting studies for expanded systematic review.

\section{Chapter Two}

Figure 2.1 Total effect of $X$ on $Y$ model and simple mediation model illustrated.

\section{Chapter Three}

Figure 3.1 Mediation model of the indirect effect of PTSS at time 2 on the relationship between rumination at time 2 and PTG at time 2 .

Chapter Four

Figure 4.1 Path illustration of key findings pertaining to social support, functioning and PTG.

Figure 4.2 Path illustration of key correlational findings pertaining to psychological adjustment and PTG.

Figure 4.3 Path illustration of key correlational findings pertaining to cognitive processing factors and PTG. 


\section{List of Appendices}

\begin{tabular}{ll} 
Appendix A & Search terms for initial systematic review \\
Appendix B & Search terms for expanded systematic review \\
Appendix C & Information sheet (version 4) \\
Appendix D & Cover letter \\
Appendix E & Information sheet (version 5) \\
Appendix F & Consent form (version 3) \\
Appendix G & Consent form (version 4) \\
Appendix H & REC approval letter \\
Appendix I & Approval process flowchart \\
Appendix J & Sponsorship letter \\
Appendix K & R\&D approval letter \\
Appendix L & Amendment (summary of changes) \\
Appendix M & Amendment approval letter \\
Appendix N & Lone worker policy \\
Appendix O & Stroke survivor survey \\
Appendix P & Skewness and kurtosis exceptions to the acceptable range \\
Appendix Q & Consultation with statistician \\
Appendix R & Formula used to calculate t-tests \\
\hline
\end{tabular}




\title{
Chapter One
}

\author{
Introduction
}

\subsection{Focus of the thesis}

Stroke is the second leading cause of death worldwide (WHO, 2014) and for those who survive, the experience can be traumatic (Stroke Association, 2015). A stroke can be construed as bearing all the hallmarks of a traumatic event given that it is typically unexpected, uncontrollable and potentially life-threatening (Field et al. 2008). Despite the sudden onset of stroke, the consequences can be long-term and far-reaching (Adamson et al. 2004). Stroke survivors often experience emotional regulation difficulties following stroke and are frequently diagnosed with anxiety and depression (Ayerby et al. 2013; Campbell Burton et al. 2013). It has also been reported that approximately $10-31 \%$ of stroke survivors experience posttraumatic stress symptoms after stroke (Bruggimann et al. 2006; Merriman et al. 2007; Sembi et al. 1998). However, less is known about the other ways in which stroke survivors psychologically adjust and adapt following stroke.

The literature on psychological adjustment to traumatic events has largely focused on negative outcomes such as distress and lower quality of life (Frazier \& Kaler, 2006). However, a growing body of literature shows that trauma survivors report positive changes across several domains such as beliefs, priorities and relationships with others following exposure to trauma (Park \& Helgeson, 2006). These positive changes are typically termed posttraumatic growth (PTG) in the literature. Theoretical models have been developed which account for the mechanisms of change underpinning PTG (Tedeschi \& Calhoun, 2004; Schaefer \& Moos, 1992). Although PTG has been investigated in a range of traumata (e.g. cancer (Sears et al. 2003), political prisoners (Maercker, 1999)), there has been limited research investigating the role of PTG in stroke survivors (Gangstad et al. 2009; Gillen, 2005; Kuenemund et al. 2014). Theoretical models of PTG (Tedeschi \& Calhoun, 1995, 2004; Schaefer \& Moos, 1992) which 
conceptualise PTG as an outcome of the struggle with a traumatic event provide a useful framework for considering the mechanisms of PTG in stroke survivors. This study aims to investigate whether PTG occurs in stroke survivors over time and whether there are particular variables, linked to relevant theoretical models, which are predictive of PTG in stroke survivors.

\subsection{Definitions of key terminology}

\subsubsection{Stroke}

In this thesis, the term 'stroke' is used to refer to a clinical syndrome characterised by 'rapidly developing clinical signs of focal (or global) disturbance of cerebral function with symptoms lasting 24 hours or longer or leading to death, with no apparent cause other than of vascular origin' (WHO, 1988).

Approximately $85 \%$ of strokes are caused by a blockage in the blood supply to the brain and classified as ischaemic stroke, whereas about $15 \%$ are caused by a major blood vessel rupturing and bleeding into the brain and classified as haemorrhagic stroke (Royal College of Physicians, 2012). This thesis will use the term 'stroke' to refer to both ischaemic and haemorrhagic type strokes.

\subsubsection{Stroke Survivors}

The term 'stroke survivor' in this thesis refers to any individual who has been diagnosed with a stroke (see section 1.2.1.) and survived. Other terms used in the literature to describe this clinical group, such as 'stroke patient' and 'stroke service user', will be subsumed under the term 'stroke survivor' in this thesis.

\subsubsection{Posttraumatic growth}

The term posttraumatic growth (PTG) is used in this thesis to refer to the positive changes that an individual can experience in response to a stressful or traumatic experience (Tedeschi \& Calhoun, 1995). There are many terms used in the literature which appear to be 
synonymous with PTG, some of which include: 'benefit finding', 'meaning-making', 'adversarial growth', 'self-transformation', 'thriving' and 'stress-related growth' (Zoellner \& Maercker, 2006). Although it is recognised that some of the aforementioned terms could be conceptualised as standalone constructs, the term PTG will subsume these variant terms when evaluating literature relevant to this thesis, unless otherwise stated.

More specifically, the term PTG is used to describe the psychological growth which can occur following a traumatic experience as opposed to any given stressful experience. In this thesis, PTG refers to the enduring changes which can follow a struggle with a traumatic experience (Tedeschi \& Calhoun, 1995, 2004). The term denotes the emotional and cognitive processing of trauma, as well as the potential for growth following trauma.

\subsubsection{Traumatic}

In this thesis, the term 'traumatic' is used to refer to the set of circumstances which can lead to an individual's adaptive resources and their way of understanding the world and their place in it being significantly challenged (Janoff-Bullman, 1992; Tedeschi \& Calhoun, 2004). Other terms commonly used in the literature, such as 'crisis' and 'highly stressful event', will be subsumed under the term 'traumatic' unless otherwise stated.

The term 'traumatic' is used in a less restrictive way in the PTG literature relative to the posttraumatic stress disorder (PTSD) literature. In the PTSD literature, the term 'traumatic' is typically used to refer to the stress response caused by events which cause actual or threatened death or serious injury (APA, 1994). However, the term 'traumatic' is used in this thesis to refer to events or sets of circumstances which may or may not threaten a person's mortality which is akin to its usage in the PTG literature. 


\subsection{Why study stroke survivors?}

\subsubsection{Epidemiology of stroke}

Every three minutes and 27 seconds, a person has a stroke in the UK (Stroke Association, 2015; Townsend et al. 2012). Between 2005 and 2014, the prevalence rate of stroke in the UK increased by $26 \%$ (Health and Social Care Information Centre, 2014; StatsWales, 2014). Of the 152,000 people a year who experience a stroke in the UK (Townsend et al. 2012), 1 in 8 are likely to die within 30 days and 1 in 4 are likely to die within a year (Office of National Statistics, 2014). Stroke is the second leading cause of mortality worldwide causing 6.7 million deaths each year (WHO, 2014) and the third leading cause of mortality in the UK (Office of National Statistics, 2014). Stroke causes about $6 \%$ of all male deaths in UK and $8 \%$ of all female deaths in the UK (Office of National Statistics, 2014). Of note, stroke mortality rates in the UK have decreased by 46\% from 1990 to 2010 (Feigin et al. 2013).

It has been estimated that there are about 1.2 million stroke survivors living in the UK. Incidence rates for stroke in the UK are reported in the literature as ranging from 115 per 100,000 population to 150 per 100,000 (Feigin et al. 2013; Wang et al. 2013). Approximately 3 in 10 stroke survivors will go on to have a further stroke or TIA (Mohan et al. 2011). It has been reported that between $25 \%$ and $33 \%$ of all strokes are recurrent strokes (Burn et al. 1994; Furie et al. 2011; Giles \& Rothwell, 2007).

In terms of demographic variables, age is considered as the single most important risk factor for stroke (Scarborough et al. 2009). About $26 \%$ of all strokes in the UK occur in those under the age of 65 (Royal College of Physicians, 2012). The risk of having a stroke doubles every decade after the age of 55 (Brown et al. 1996). By the age of 75, 1 in 5 women and 1 in 6 men will have had a stroke (Seshadri et al. 2006). In terms of ethnicity, the evidence suggests that people who are of black or South Asian ethnicity are at a higher risk of stroke compared to Caucasians (Wang et al. 2013). In terms of social deprivation, the evidence suggests that people from the most economically deprived areas of the UK are about twice as likely to have a stroke than those from the least deprived areas (Cox et al. 2006; Public Health England, 2015). 
Stroke is the largest cause of complex disability in the UK relative to any other condition (Adamson et al. 2004). About half of all stroke survivors experience residual disability following stroke (Royal College of Physicians, 2012). About 37\% of stroke survivors who are discharged from hospital require help with activities of daily living (Adamson et al. 2004).

\subsubsection{The impact of stroke on public health}

It is estimated that the health and social care costs of stroke in the UK are approximately $£ 4.38$ billion a year; this rises to $£ 9$ billion a year if informal care costs and those to the wider economy are included (Saka et al. 2009). Following the National Audit Report (2005) in the UK, stroke care became a priority with the development of the National Stroke Strategy (2007) and NICE guidelines which set out the best practice guidelines for quality stroke care. More recently, the psychological aspects of care and wellbeing are being recognised at a policy level (e.g. NHS improvement: Psychological care after stroke, 2011).

\subsubsection{The impact of stroke at an individual level}

Stroke survivors can experience a range of both short-term and long-term consequences following stroke (Adamson et al. 2004). A stroke can cause physical difficulties such as paralysis or muscle weakness, sensory loss, fatigue and continence difficulties; speech and language difficulties such as swallowing and communication; cognitive difficulties related to vision, spatial awareness, memory and reasoning; as well as psychological difficulties (Burton, 2000). Post-stroke sequelae and complex care needs can have a profound impact on an individual's psychological well-being, quality of life, relationships, finances and future health (Glass \& Maddox, 1992; Stroke Association, 2015).

\subsubsection{Psychological Adjustment following Stroke}

The literature on psychological reactions to traumatic events has traditionally focused on outcomes such as distress and lower quality of life (Frazier \& Kaler, 2006). Kaufman and 
Becker (1991) conceptualise stroke survivors' experience of stroke as an 'identity assault' on their 'taken for granted body' which alters their 'natural right sense of self'. In this way, they posit that having a stroke disturbs one's ability to engage in previously valued social roles which can be further complicated by systemic factors such as family dynamics. Moreover, Glass and Maddox (1992) have conceptualised psychological adjustment post-stroke in more explicit cognitive terms. They suggest that stroke affects one's 'assumptive world' and 'lifespace' whereby identity, self-concept and role capability are all challenged.

It is estimated that over half of stroke survivors experience depression at some point after stroke (Ayerbe et al. 2013) and that approximately a third of stroke survivors experience poststroke depression at any one time (Hackett et al. 2005). Other common emotional changes following stroke can include anxiety, emotionalism, anger and personality changes such as low self-esteem (House et al. 1991; Stone et al. 2004). The literature suggests that reported rates of anxiety in studies of stroke survivors are likely to underrepresent the extent of the problem (Campbell Burton et al. 2013). It has been estimated that between 18-25\% of stroke survivors experience significant anxiety within the first year post-stroke (Campbell Burton et al. 2013).

There is evidence to suggest that some stroke survivors continue to experience depression for prolonged periods post-stroke and that this can be associated with negative health outcomes (Ayerbe et al. 2013; Hare et al. 2006; Murray et al. 2003). Following a systematic review and meta-analysis, Ayerbe et al. (2013) found a prevalence rate of $29 \%$ for depression post-stroke which remained stable for up to ten years post-stroke with a cumulative incidence of $39-52 \%$ within five years post-stroke. They also found that the rate of recovery from depression which was identified a few months post-stroke ranged from 15 to $57 \%$ within the first year post-stroke. However, it is increasingly recognised that stroke survivors may experience a range of emotional responses when adjusting to life after stroke and there is evidence that some may perceive benefits post-stroke (Gangstad et al. 2009; Gillen, 2005; Kuenemund et al. 2014).

In the literature, it is somewhat unclear how the outcome of psychological adjustment following stroke can best be measured. More specifically, it is disputed whether psychological adjustment following stroke constitutes the absence of what could be termed negative 
outcomes (e.g. PTSD, depression), or if it involves the presence of positive outcomes (e.g. psychological wellbeing, PTG) (Wortman, 2004). In addition, it is also unclear whether psychological adjustment is most suitably measured using perceived or objective measures of adjustment, whether the perception of benefit translates into objective life changes and finally, whether PTG is related to psychological adjustment (Park, 2004).

\subsubsection{Outcomes following Stroke}

Given the decreasing mortality rate following stroke in the UK, more people are surviving and living with stroke than ever before (Feigin et al. 2013). It is reported that stroke survivors who are inpatients on stroke specific units have better outcomes in terms of mortality, functional independence and discharge home relative to stroke survivors who are inpatients on other types of wards (Stroke Unit Trialists' Collaboration, 2007).

Although stroke survivors often benefit from rehabilitation, many are left with residual longterm difficulties (Hillis \& Tippett, 2014). Research also suggests that depression can impact on a stroke survivor's ability to make rehabilitation gains (West \& Bernhardt, 2011). Stroke survivors often make significant improvements in their recovery in the first 6 months, but recovery is an ongoing process which may continue for years (Pollack et al. 2002). About one third of stroke survivors experience significant long-term disability on discharge from hospital (Adamson et al. 2004). Long-term disability post-stroke may stem from difficulties relating to cognition, mobility, communication, continence and psychological well-being which may range in severity from mild to severe (Adamson et al. 2004). Although it was previously thought that most recovery of function occurs within three months post-stroke (Skilbeck, 1983), emerging evidence suggests that stroke survivors can make significant gains in recovery many years after stroke (Smania et al. 2010). In this way, it appears that there are no predetermined trajectories post-stroke and the process of recovery post-stroke is individual. 


\subsection{Posttraumatic Growth}

\subsubsection{Conceptualisation of posttraumatic growth}

The concept of suffering leading to psychological growth has been an integral part of philosophy, literature and spiritual traditions spanning human history (e.g. Caplan, 1964; Frankl, 1963; Kierkegaard, 1983; Nicholls, 1955; Yalom, 1980). More recently, the clinical psychology literature has reflected a growing interest in the transformative effects of trauma and efforts have been made to empirically investigate this concept (e.g. Hefferon et al. 2009; Helgeson et al. 2006; Joseph \& Linley, 2006; Tedeschi \& Calhoun, 1996; Zoellner \& Maercker, 2006). This concept of psychological growth following suffering is most commonly referred to as posttraumatic growth in the literature and was first coined by Tedeschi and Calhoun (1995).

Posttraumatic growth has been defined as an individual's experience of significant positive change resulting from the struggle with a major life crisis (Calhoun et al. 2000, p.521). Tedeschi and Calhoun (2004) postulate that PTG can lead to: 'an increased appreciation for life in general, more meaningful interpersonal relationships, an increased sense of personal strength, changed priorities, and a richer existential and spiritual life'.

Importantly, posttraumatic growth refers to an experience of improvement and profound positive change which goes beyond pre-trauma levels of psychological functioning (Tedeschi \& Calhoun, 2004; Silva et al. 2011). According to Tedeschi \& Calhoun (2004), 'it is the individual's struggle with the new reality in the aftermath of the trauma that is crucial in determining the extent to which posttraumatic growth occurs'. In this way, it is theorised that individuals who do not experience a struggle following trauma are less likely to experience posttraumatic growth.

Tedeschi and Calhoun (2004) account for the role of pre-existing personality traits in their conceptualisation of PTG and posit that qualities such as resilience and optimism make an individual more likely to endure trauma but do not necessarily predict growth. Critically, it is not only the threat to an individual's assumptive world (e.g. assumptions about the predictability and controllability of the world) but the accompanying emotional distress which facilitates the transformative experience of growth (Tedeschi \& Calhoun, 2004). In this way, it is proposed that growth requires a process of cognitive processing and emotional 
engagement which co-exists alongside the residual emotional distress of the trauma (Tedeschi \& Calhoun, 2004).

\subsubsection{Traumatic events and PTG}

In the research literature, PTG has been investigated using a broad range of traumata such as military combat (Fontana \& Rosenheck, 1998), war (Ai et al. 2007), rape (Thompson, 2000), terrorist led events (Woike \& Matic, 2004), spinal cord injury (McMillen \& Cook, 2003) and AIDS/HIV (Seigel et al. 2005). Traumata vary widely in terms of event-related factors such as severity, proximity to and exposure to event, extent of loss, duration, predictability and suddenness of onset (Schaefer \& Moos, 1998, p.107). The literature suggests that irrespective of event-related factors, response to trauma and psychological adaptation varies widely and it is acknowledged that such responses are likely to mediate the development of PTG (Schaefer \& Moos, 1998; Tedeschi \& Calhoun, 2004).

Following a traumatic event, an individual may experience 'disruptions in significant relationships, challenges to their values and beliefs, role changes and new demands' (Schaefer \& Moos, 1992, p.150). It is theorised that such experiences can lead to a richer appreciation for life, broader priorities, more compassion for others, closer relationships, new coping skills, as well as access to new personal and social resources (Schaefer \& Moos, 1992;Tedeschi \& Calhoun, 1995, 2004). According to the literature, the trajectory toward PTG following a traumatic event does not follow a uni-directional or fixed time course (Schaefer \& Moos, 1998, Tedeschi \& Calhoun, 2004). In spite of whether PTG is conceptualised as an outcome or a process, the transformative nature of PTG generally is viewed in the literature as arising from a personal struggle (Tedeschi \& Calhoun, 1996). In this way, it is increasingly recognised that PTG often co-exists alongside emotional distress for at least part of an individual's PTG journey (Tedeschi \& Calhoun, 2004). 


\subsubsection{Models of PTG}

Although some theorists have conceptualised PTG as a process borne out of attempts to cope following trauma (e.g. Park \& Folkman, 1997), the most dominant models in the literature conceptualise PTG as an outcome (Schaefer \& Moos, 1992; Tedeschi \& Calhoun, 1995, 2004). Such models of PTG also propose mechanisms underpinning the transformational change associated with PTG and will be explored in further detail below: Schaefer and Moos (1992) model of life crises and personal growth, and Tedeschi and Calhoun $(1995,2004)$ revised model of posttraumatic growth.

\subsubsection{Model of life crises and personal growth (Schaefer \& Moos, 1992)}

According to Schaefer and Moos (1992), personal system factors, environmental factors and event-related factors influence the development of PTG through the use of cognitive appraisal processes and coping resources following exposure to a traumatic event (see Figure 1.1). They propose that the personal system includes socio-demographic characteristics and personal resources such as self-efficacy, resilience, optimism, self-confidence, an easy-going disposition, motivation, health status and prior experience. Environmental factors include personal relationships, support from family, friends and social environment as well as financial resources and other aspects of the living situation. Event-related factors include the effects of the severity, duration and timing of the life crisis and its scope on the individual. They highlight the importance of approach coping (i.e. rational analysis of the problem, positive reappraisal, active coping) for growth to occur as opposed to avoidance coping (i.e. denial, minimising the problem).

According to this model, each factor makes a unique contribution to PTG and the model is dynamic in the sense that the effects of different factors can be bi-directional. This model predicts that the severity of the experience facilitates growth, but only when there is a successful outcome in the face of poor prognosis. It is also theorised that severe events such as those involving disfigurement and loss of bodily function (e.g. some cases of stroke) are associated with less growth. The model also makes predictions about how environmental factors and personal factors influence other factors. For example, it is predicted that social support indirectly effects the coping style used and the perception of trauma severity. 


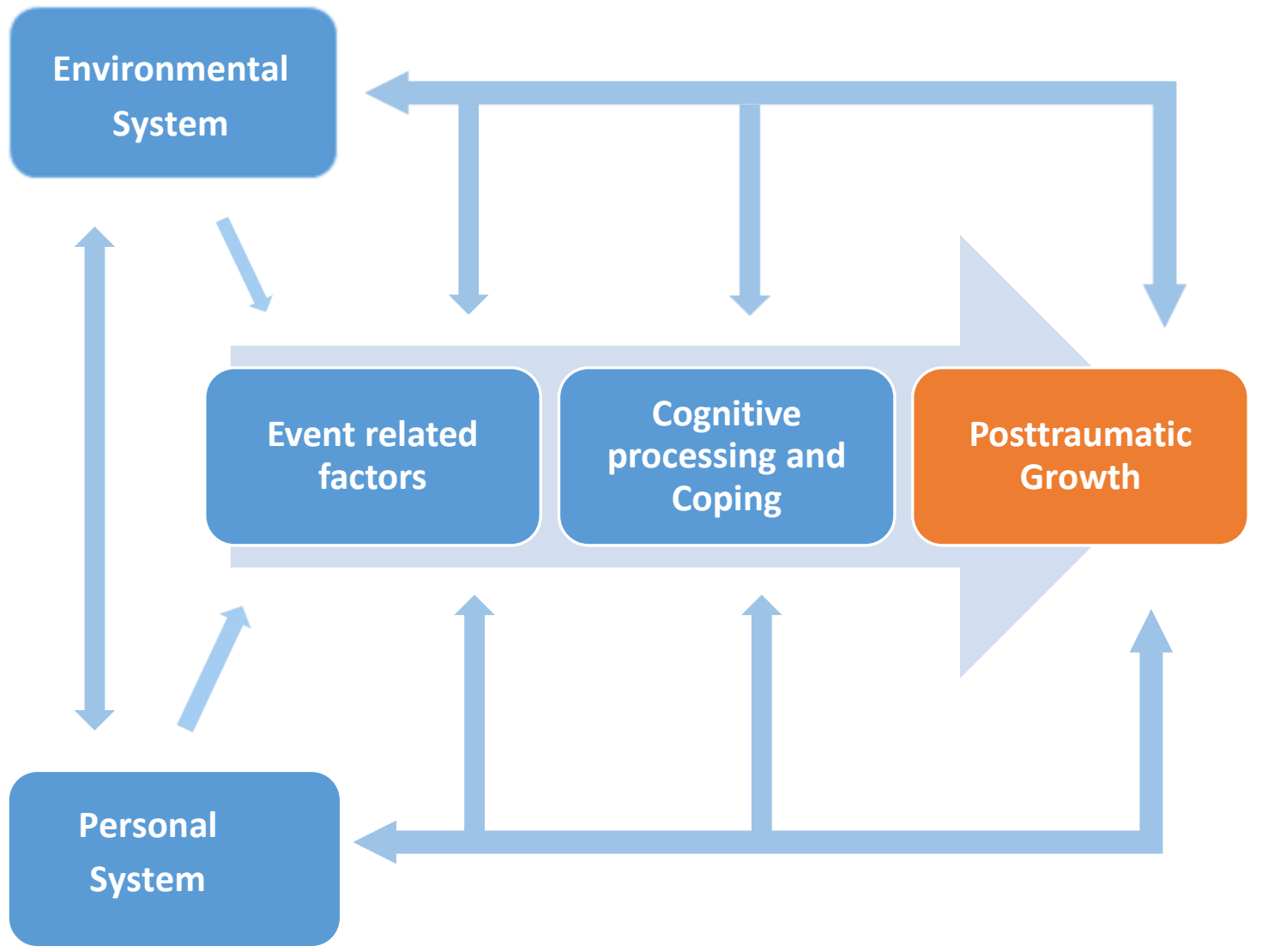

Figure 1.1 Model of life crises and personal growth (Schaefer \& Moos, 1992) (Adapted version).

1.4.3.2 Revised model of PTG (Tedeschi \& Calhoun, 1995, 2004)

Tedeschi and Calhoun's model of PTG was first developed in 1995 and later revised in 2004 to further elucidate the mechanisms by which growth occurs. This model is more comprehensive and cognitively orientated than that of Schaefer and Moos (1992) (Joseph \& Linley, 2006). According to this model, traumatic events facilitate transformative change or PTG when the trauma survivor experiences a 'crisis' which leads to a process of reappraising personal goals and worldviews (see Figure 1.2). They theorise that this 'crisis' is characterised by emotional distress, recurrent rumination about the trauma and associated factors, as well as efforts to reduce feelings of distress. This process of rumination can be 'constructive or destructive depending on whether rumination supports continued negative thoughts or 
emotions or helps move the person toward problem solving or finding meaning' (Calhoun et al. 2010). Tedeschi and Calhoun (2004) hypothesise that when trauma survivors experience success in their efforts to cope, rumination becomes more deliberate and effortful. In this way, rumination serves as a form of constructive cognitive processing by enabling a trauma survivor to find meaning and make positive re-appraisals. According to this model, it is hypothesised that PTG can lead to changes across a range of domains such as personal beliefs, life goals, perception of relationships and identity.

In terms of the role of automatic rumination, Tedeschi and Calhoun (2004) have hypothesised that it involves spontaneous, frequent and intrusive qualities which are somewhat consistent with the re-experiencing and avoidance characteristic of PTSD (Joseph \& Linley, 2006). Tedeschi and Calhoun (2004) propose that such cognitive processes in the acute phase posttrauma represent efforts of re-integration of experience with identity and core beliefs.

This model also accounts for the role of individual differences, social support and coping in facilitating PTG. According to Tedeschi and Calhoun (2004), social support provides ways for trauma survivors to learn new ways of coping with trauma but also facilitates a sense of psychological safety which supports the process of deliberate rumination. Acceptance coping is also viewed as integral to enabling trauma survivors to re-define achievable personal goals (Calhoun et al. 2000). This model borrows from the work of Janoff Bulman (1992) in the sense that traumatic events are theorised as leading to a trauma survivor challenging their world view and their locus of control. Of note, the model also posits that the more an individual's world views and assumptions are challenged, the more likely they are to experience PTG.

\subsubsection{Summary of the models}

Both models (Schaefer \& Moos, 1992; Tedeschi \& Calhoun, 1995; 2004) are comprehensive and inclusive of the distal and proximate predictors of PTG accounted for in the literature (see Zoellner \& Maercker, 2006). However, there is a need for the use of more consistent terminology which would allow for more comparisons and theoretical specifications to be made. Due to the over inclusive nature of some of the predictors of PTG, both models are difficult to test empirically. However, the models explored do appear to serve as a useful 
heuristic for guiding research questions and as a way of deriving more empirically testable theoretical predictions.

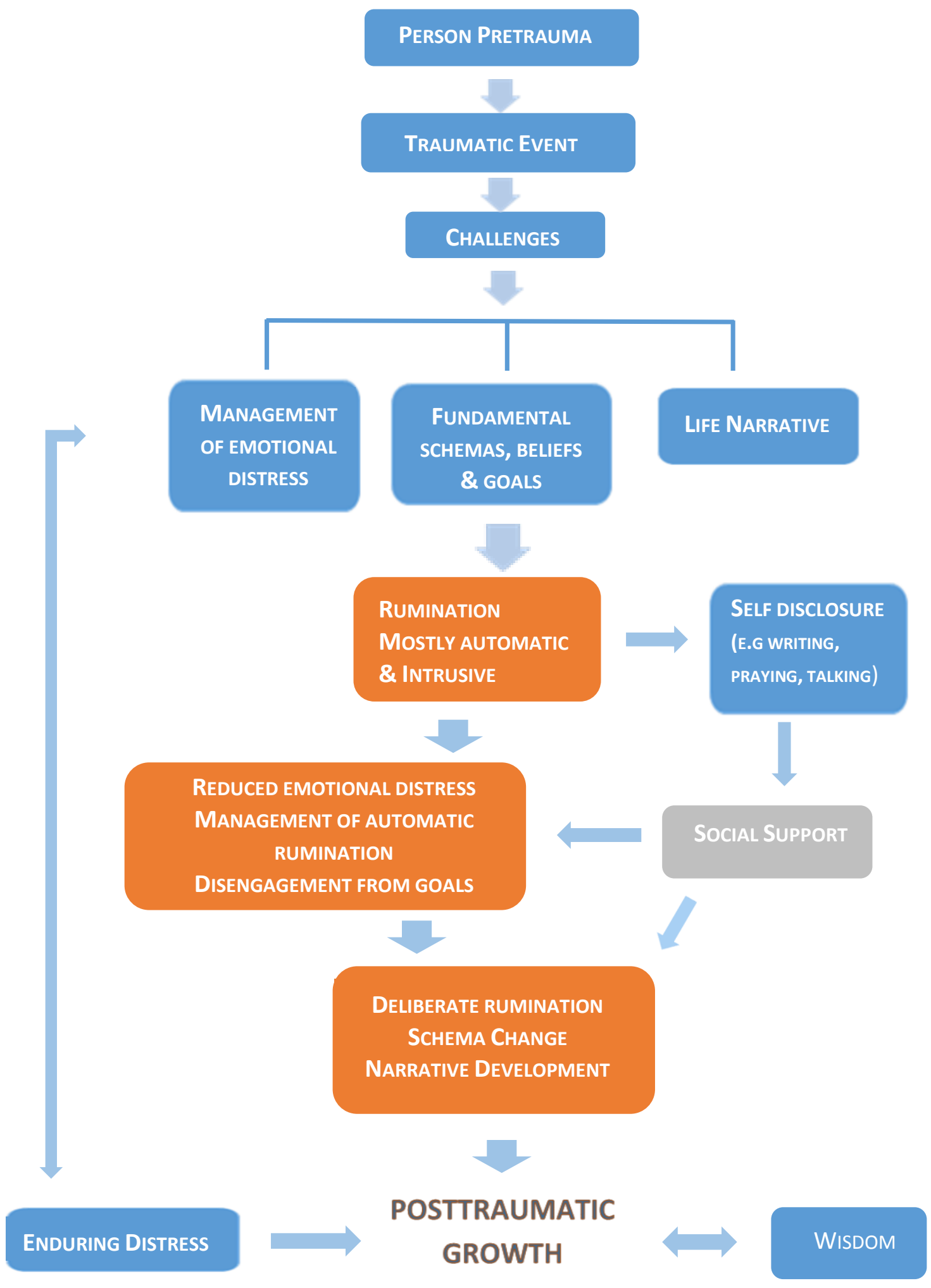

Figure 1.2 Revised model of PTG (Tedeschi \& Calhoun, 1995, 2004) (Adapted version). 
Both models also account for the role of social support in enabling a person to begin their search for meaning. Similarly, both models account for the role of personality variables such as extraversion and openness to experience in facilitating PTG. In terms of denial or avoidance coping, both models posit that such coping is not conducive to PTG.

One of the primary differences between both models is how they predict event severity impacts on growth. Schaefer and Moos (1992) propose that PTG occurs when a person survives a trauma despite a poor prognosis, whereas Tedeschi and Calhoun (2004) propose that PTG can occur when faced with a poor prognosis post-trauma. More generally, Schaefer and Moos (1992) consider multiple factors as directly contributing to PTG whereas Tedeschi $\&$ Calhoun (2004) view rumination, social support and acceptance coping as most predictive of PTG. Schaefer and Moos (1992) also propose that approach or active coping is central to PTG, whereas Tedeschi and Calhoun (2004) emphasise the role of deliberate rumination.

\subsubsection{Empirical support for models from the literature}

Few studies have explicitly tested the theoretical predictions made by the models of PTG proposed by Schaefer and Moos (1992) and Tedeschi and Calhoun (2004). More recently, the emerging literature is providing evidence which broadly support the theoretical predictions made by Tedeschi and Calhoun (2004). For example, using a sample of myocardial infarction survivors, Senol-Durak and Ayvasik (2010) found that social support was directly associated with PTG and also found that rumination and emotional coping were most predictive of PTG. Similarly, Hallam and Morris (2014) found that deliberate rumination predicted PTG and mediated the relationship between social support and PTG which provides support for the model proposed by Tedeschi and Calhoun (2004). Studies of bereaved parents have also found that PTG is associated with cognitive and emotional processing within the context of supportive relationships (Hogan \& Schmidt, 2002; Znoj \& Keller, 2002).

In terms of the model of PTG proposed by Schaefer and Moos (1992), there are mixed findings regarding the association between PTG and avoidance coping (Zoellner \& Maercker, 2006), as well as age (Helgeson et al. 2006). However, studies which have found an association between PTG and positive reappraisal (e.g. Sears et al. 2003), active coping (e.g. Dekel et al. 
2011) and social support (e.g. Hallam \& Morris, 2014) provide evidence in support of this model.

\subsubsection{Factors associated with PTG in the literature}

Although PTG has been associated with a range of factors in the general literature (Helgeson et al. 2006; Linley \& Joseph, 2004; Zoellner \& Maerker, 2006), less is known about the factors associated with PTG in the stroke-specific literature. This section will explore key findings from both the stroke-specific and general literature using the following themes: relationships, psychological adjustment, cognitive processing and event-related factors. In addition, the key findings discussed will be appraised in light of the dominant theoretical frameworks used to conceptualise PTG (Tedeschi \& Calhoun, 1996, 2004; Schaefer \& Moos, 1998).

\subsubsection{Relationships}

Social support includes the support that a person receives from friends, family and significant others (Zimet et al. 1988). Tedeschi and Calhoun (2004) suggest that social support facilitates PTG by helping the person to gain access to models of schema change but also to provide comfort, reduce emotional distress and allow for deliberate rumination. Schaefer and Moos (1998) suggest that social resources promote growth through facilitating the development of effective coping strategies. There is evidence to suggest that social support is associated with the development of PTG in a range of traumata (Park, 2010). Social support is associated with more successful rehabilitation outcomes in myocardial infarction survivors and better psychological adjustment in breast cancer survivors (Moos, 1985). Although there are no known studies examining whether social support is associated with PTG in stroke survivors, one study has found a positive relationship between social support and PTG in stroke carers (Hallam \& Morris, 2014).

The distinction between perceived and actual social support is rarely made in the literature, nor is the role of individual and systemic factors (e.g. seeking out support versus readily accessible social support). However, it is theorised that irrespective of objective realities, it is the perception of social support that is integral to PTG (Tedeschi \& Calhoun, 2004). Of note, 
it is also unclear whether social support interventions in stroke such as peer support are related to the development of PTG (Schroevers et al. 2014).

\subsubsection{Psychological adjustment}

Some theorists have conceptualised PTG as a coping strategy (Park \& Folkman, 1997) whereas others have framed it as an outcome (e.g. Tedeschi \& Calhoun, 2004); it is the latter construction of PTG that forms the basis of this thesis. In this way, it is theorised that PTG occurs after a 'struggle' which involves a process of coping and engaging with emotionality following a traumatic event (Schaefer \& Moos, 1992; Tedeschi \& Calhoun 1995, 2004). There is evidence to suggest that a range of emotional regulation difficulties such as anxiety (Ayerby et al. 2013), depression (Campbell Burton et al. 2013) and PTSD (Sembi et al. 1998) can occur following stroke. According to Tedeschi and Calhoun (2004), emotional regulation difficulties characterise the 'struggle' following a traumatic event and can continue to co-exist, to a greater or lesser degree, alongside PTG (Morrison et al. 2005). Conflicting findings in the general literature regarding the relationship between psychological factors and PTG appear to be mirrored in the emerging literature on PTG in stroke survivors (Zoellner \& Maercker, 2009). For example, Gangstad et al. (2009) found a negative association between depression and PTG, whereas Kuenemund et al. (2014) found that stroke survivors reported significantly higher depression and lower satisfaction with life compared to controls.

The process of coping, or efforts made to reduce the stressfulness of an event (Lazarus \& Folkman, 1986), can be categorised as either acceptance coping (i.e. consciously attempting to accept what has happened), denial coping (i.e. avoiding thinking about an event) or ruminative coping (i.e. attempting to make sense of an experience). There is evidence to suggest that PTG can be positively associated with problem-focused, active coping (Armeli et al. 2001; Maercker \& Langner, 2001). Schaefer and Moos (1992) argue that active rather than avoidance coping is involved in the development of PTG, whereas Tedeschi and Calhoun $(1995,2004)$ propose that deliberate rumination facilitates opportunities for positive reappraisal and growth following trauma.

Research suggests that PTG is positively associated with acceptance coping style (Park et al. 1996), active coping (Morris et al. 2007), positive reappraisal coping (Gangstad et al. 2009) 
and deliberate rumination (Taku et al. 2009). Interestingly, studies have also found that denial coping is associated with PTG in stroke survivors (Gangstad et al. 2009) and stroke carers (Hallam \& Morris, 2014). Similarly, studies of cancer survivors have also found that a degree of adaptive coping and denial coping appear to predict PTG (Lelorain et al. 2010).

\subsubsection{Cognitive processing}

According to Tedeschi \& Calhoun (2004), cognitive processing factors facilitate the development of PTG, some of which include: openness to new experience, hardiness and sense of coherence, dispositional optimism, and internal locus of control. Tedeschi and Calhoun (2004) hypothesise that the mechanism through which growth occurs involves a process of deliberate rumination which leads to acceptance and positive re-appraisal over time. There is evidence to suggest that acceptance, planning, rumination, self-blame and other-blame are associated with PTG in a range of trauma survivors (Helgeson et al. 2006). In the stroke literature, Gangstad et al. (2009) found that positive cognitive restructuring, downward comparsion, resolution and denial were associated with PTG. Additionally, Kuenemund et al. (2014) found that stroke survivors integrated their experience of stroke into their identity to a greater extent than controls who identified a stressful life event. However, the literature is less clear about whether PTG involves challenging high-order goals and beliefs (Zoellner \& Maercker, 2006).

\subsubsection{Other factors}

According to the literature, other factors worthy of consideration include age (Widows et al. 2005), gender (Park et al. 1996), personality (Updegraff et al. 2002) and event-related factors such as event severity, duration, time since event and event centrality (Tedeschi \& Calhoun, 1996). Studies have found that age and lower educational level are negatively associated with PTG (Widows et al. 2005), while others have found that females are more likely to experience PTG (Park et al. 1996). However, the pattern of findings regarding such factors and PTG appears to be largely inconsistent in the literature (Zoellner \& Maercker, 2006).

In a study of stroke survivors, Gangstad et al. (2009) found that cognitive processes such as downward comparisons and resolution moderated the relationship between time and PTG. This supports the theoretical prediction made by Tedeschi and Calhoun (2004) that the 
development of PTG involves engaging in deliberate rumination over time. Although studies suggest that PTG occurs with time (e.g. Cordova et al. 2001; Sears et al. 2003), few studies have examined the temporal nature of PTG longitudinally (e.g. Dekel et al. 2012). One study of stroke survivors has found that event centrality is key to integrating the experience of trauma into one's identity (Kuenemund et al. 2014), but it is unclear whether the role of event centrality changes over time. The literature suggests that the nature of PTG does not differ based on the type of traumatic event (Zoellner \& Maercker, 2006).

\subsubsection{Criticisms of PTG}

There have been many criticisms levelled not only at specific models of PTG, but also at the concept of PTG itself. One of the primary criticisms of PTG is that it is illusory and serves a defensive function and provides a way of avoiding engagement with one's emotional experience following a trauma (e.g. Aldwin \& Levenson, 2004; Campbell et al. 2004). Wortman (2004) has questioned the validity of measures used to capture PTG in the literature and suggests that prospective research is required to determine whether reports of positive change following trauma are veridical. The contradictory findings regarding PTG and its adaptive significance are often cited in support of this argument (Zoellner \& Maercker, 2006). However, Calhoun and Tedeschi $(2004$, p.95) contend that there are individual differences whereby a trauma survivor's 'struggle' is most likely characterised by a mixture of positive and negative experiences over time: '... different trajectories are possible that will sustain and enhance a posttraumatic growth perspective in some trauma survivors, whereas other may find this view fading over time'. They also argue that downward comparisons do not explain PTG despite the evidence which suggests that PTG is often corroborated by others within one's social network (Park et al. 1996; Weiss, 2002).

Some have argued that there may be a social desirability bias inherent in reports of PTG (e.g. Zoellner \& Maercker, 2006; McFarland \& Alvaro, 2000) despite the use of a measure of social desirability by Tedeschi and Calhoun (1996) when validating the Posttraumatic Growth Inventory (PTGI) measure of PTG. The literature appears to support the theoretical prediction made by Tedeschi and Calhoun (2004) that both positive and negative feelings can co-exist 
during the development of PTG and it is likely that this accounts for the apparent 'illusory' nature of PTG (Zoellner \& Maercker, 2004). Tedeschi \& Calhoun (2004) argue that rather than the emotionality of trauma making individuals more prone to biases such as distortion and defensive functioning, it can make individuals less prone to such biases. They posit that individuals might be able to better integrate the emotionality of the trauma due to their baseline level of emotional wellbeing and existing coping resources. Calhoun and Tedeschi (2004) found that although young adults were more likely to report change regardless of their circumstances, the perception of change was stronger for those who had experienced trauma. Furthermore, there is some evidence to suggest that perception of growth can be associated with objective measures of psychological adjustment (e.g. Goodhart, 1985) but further research is required to determine the role of social desirability and the potential for illusory PTG.

PTG has also been criticised on the grounds of cultural bias due to the ways in social and cultural factors, at a distal and proximate level, are likely to influence how responses to traumatic events are conceptualised (Park, 2004). Cultural factors inform how life-threatening experiences are socially constructed, how trauma is defined, what constitutes adaptive and maladaptive functioning, as well as how norms and expectations regarding coping and resilience are defined (Wilson \& So-Kum Tang, 2007). Although the literature supports the cross-cultural validity of PTG as a concept, some argue that it is debatable whether the very concept of PTG subserves the assumptions underpinning a Western individualistic society (Splevins et al. 2010). Nevertheless, empirically grounded models of PTG account for the role of proximate and distal sociocultural factors (e.g. social support and national identity respectively) and explain how these factors contribute to PTG (e.g. Tedeschi \& Calhoun, 2004).

\subsubsection{Clinical utility of PTG}

Traditionally, the literature has conceptualised psychological adjustment to traumata within a deficit oriented model focusing on the detrimental effects of trauma (Zoellner \& Maercker, 2006). This is reflective of the clinical psychology literature more generally, which has only 
recently begun to shift in focus with the advent of the positive psychology movement (Seligman \& Csikszentmihalyi, 2000). Gable and Haidt (2005) define positive psychology as 'the study of the conditions and processes that contribute to the flourishing or optimal functioning of people, groups, and institutions'. There is an emerging evidence base to suggest that key positive psychology constructs such as optimism and a sense of personal control are protective factors for both physical and mental health (Taylor et al. 2000). Gable and Haidt (2005) contend that increased understanding regarding the psychosocial factors and personal strengths which buffer against health difficulties would enable clinicians to better manage those who do suffer. Bolstered by the positive psychology literature, PTG has emerged as an area of clinical interest and empirical enquiry (Tedeschi \& Calhoun, 2004).

Clinically, there is growing evidence regarding the efficacy of integrating aspects of PTG into clinical practice (Joseph \& Linley, 2006). In a study of breast cancer survivors randomly assigned one of two interventions, Stanton et al. (2002) found that participants who wrote about their positive thoughts and feelings had fewer medical appointments three months later when compared to participants who wrote factually about their experience. Although the utility of applying aspects of PTG in a clinical setting is somewhat unclear, emerging findings (e.g. Stanton et al. 2002) support the theoretical prediction that growth arises through finding meaning post-trauma rather than as a direct result of the traumatic event itself. According to Joseph and Linley (2006), PTG can be integrated into clinical practice through increasing awareness of the potential for PTG, listening out for news of growth when working with trauma survivors and using reflective listening skills to focus on narratives of PTG during therapy. However, they emphasise that clinicians should refrain from setting PTG as a clinical outcome or implying that a lack of PTG suggests a failure to find meaning posttrauma. In this way, the literature suggests that PTG serves as a useful heuristic, conceptually, in broadening clinical perspectives regarding the possibility of psychological growth following trauma (Linley \& Joseph, 2004).

Unlike many of the traumata investigated in the PTG literature (e.g. war; Ai et al. 2007), the trauma associated with life-threatening illness is distinctive due to the health risks conferred by life-threatening illnesses such as disease recurrence (Carboon et al. 2005; Zoellner \& Maercker, 2006). Emerging findings in the literature suggest that positive emotions can serve 
a protective function in disease development (Richman et al. 2005) and can contribute to reduced mortality rates over time (Affleck et al. 1987). It is possible that the adaptation process and perception of growth may vary for different traumata and that the adaptive significance of perceived growth may vary over time. Of interest, Sears et al. (2003) found that identifying benefits post-cancer did not influence adjustment, whereas effortful frequent identification of benefits predicted future positive mood and perceived health. This finding highlights the need for further research regarding the clinical utility of PTG and its adaptive value over time.

\subsubsection{Summary of section 1.4 .}

The term posttraumatic growth (PTG) describes the positive changes which can occur following exposure to a traumatic experience (Tedeschi \& Calhoun, 2004). Studies have found evidence of PTG in survivors of different traumata (see Zoellner \& Maercker 2006). Findings suggest that irrespective of trauma severity, there is huge variance in psychological adaptation post-trauma which supports the theory that psychological response to trauma mediates the development of PTG (Tedeschi \& Calhoun, 2004). Theoretical models which offer frameworks for understanding how PTG occurs are developing an empirical grounding in the literature (Schaefer \& Moos, 1992; Tedeschi \& Calhoun, 2004). Criticisms have been levelled at the PTG literature regarding the role of social desirability and cultural bias, despite such models providing a framework for conceptualising these alleged shortcomings (e.g. Park (2004)). Research findings suggest that factors such as social support, cognitive processing, emotional processing and coping post-trauma are associated with the development of PTG (see section 1.4.4.). Of note, there is emerging evidence regarding the benefits of integrating aspects of PTG into clinical practice. Sears et al. (2005) have demonstrated the benefit of a prolonged positive intervention (i.e. identifying benefits post-cancer) in maintaining positive mood and health over time. 


\subsection{Systematic Review}

\subsubsection{Overview}

A systematic review was conducted to find and critically appraise the quality of all the studies relevant to this research study. More specifically, a systematic review was conducted in order to identify variables associated with the development of PTG which could be used to inform the hypotheses of this study. An initial systematic review was conducted which addressed the role of PTG in stroke survivors. However, due to the paucity of research in this area, an additional systematic review was conducted to address the role of PTG in a physical health condition other than stroke. The sections which follow outline the review methodology, appraise the quality of relevant studies and critically review said studies in light of the review question, for each of the systematic reviews conducted.

\subsubsection{Initial systematic review}

\subsubsection{Review Methodology}

A systematic literature search was carried out to explore the predictors of posttraumatic growth in stroke survivors. The systematic review was conducted in order to answer the following question: "What are the predictors of posttraumatic growth in stroke survivors?"

On the $23^{\text {rd }}$ of December 2014, a review of the clinical research evidence was conducted using EMBASE (1947-present), Ovid MEDLINE (R) (1946-present), PsycINFO (1806-present) and PsycArticles Full Text. The search terms focused on three key areas: 'posttraumatic growth', 'survivor' and 'stroke' (see Appendix A for a complete list of the search terms used). The search terms were combined using Boolean operators (i.e. 'and', 'or'). All abstracts and titles identified during this search were reviewed ( $N=164$ after removal of duplicates).

Studies were selected for further review if participants were stroke survivors and if posttraumatic growth was measured either quantitatively or qualitatively. Only peerreviewed, original articles, published in English between 1980 and 2014 were reviewed. Studies which included stroke survivors younger than 18 years of age and review or discussion 
papers were excluded. A flow chart illustrating the selection process for studies included in the systematic review can be found in Figure 1.3.

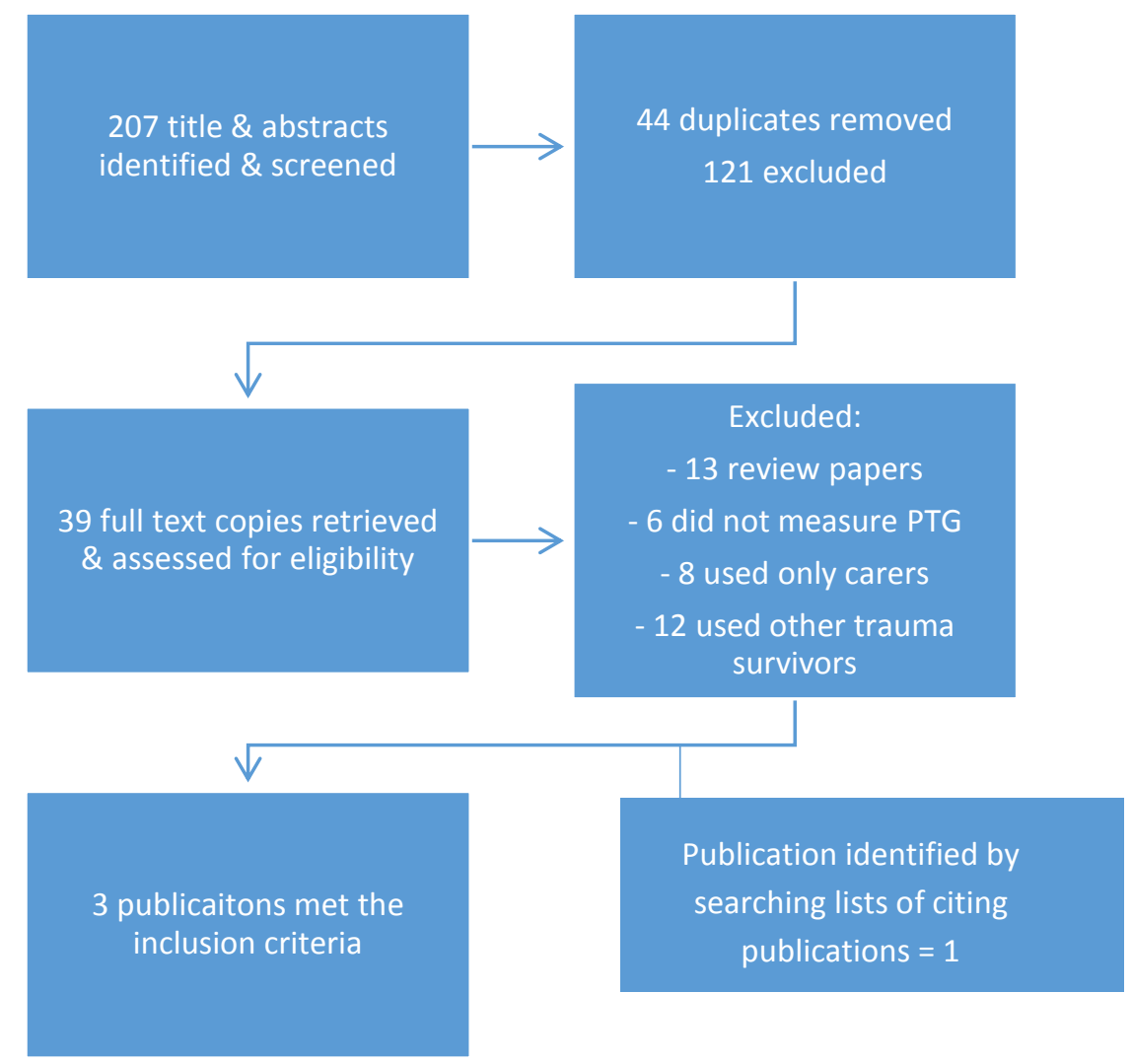

Figure 1.3 Flow chart of the procedure for selecting studies for initial systematic review

\subsubsection{Review results and quality framework}

An overview of the 3 studies which met the inclusion criteria for further review can be found in Table 1.1. The Support Unit for Research Evidence (SURE) frameworks for cross sectional/correlational studies and qualitative studies were used to evaluate the studies which met the inclusion criteria for review (see Table 1.2 and Table 1.3). The scoring guidance from the SURE quality framework was employed and supplemented with numerical coding (Cardiff University, 2012): good quality (++ / score of 2), mixed quality (+ / score of 1) and poor quality (-) or not reported (nr) (score of 0 ). Of note, the qualitative quality framework used was a revised version of the Health Evidence Bulletins Wales (HEBW) checklist and the scoring differed (i.e. yes, can't tell or no). A narrative description of the quality of the studies 
is also presented where a range of factors are considered, some of which include: samples and populations, design and recruitment, sample size, treatment of confounding variables and overall quality. 
Table 1.1 Summary of the studies which met the inclusion criteria for the initial systematic review

\begin{tabular}{|c|c|c|c|c|c|c|c|c|}
\hline Paper & Country & Sample & Method & $\begin{array}{l}\text { Measure } \\
\text { of PTG }\end{array}$ & $\begin{array}{c}\text { Other } \\
\text { Measur } \\
\text { es }\end{array}$ & $\begin{array}{c}\text { Theoretical } \\
\text { Model }\end{array}$ & Key Findings & Key Limitations \\
\hline $\begin{array}{c}\text { Gangstad } \\
\text { et al. (2009) }\end{array}$ & UK & $\begin{array}{l}\mathbf{N}=60 \\
\text { Stroke survivors } \\
\text { (Male = 34; } \\
\text { Female }=26) \\
\text { Age } \\
41-88 \text { years } \\
(\mathrm{M}=71.67 ; \\
\text { SD = 10.64) } \\
\text { Time since } \\
\text { Stroke } \\
5-99 \text { months } \\
(\mathrm{M}=32.02 ; \\
\mathrm{SD}=23.9)\end{array}$ & $\begin{array}{l}\text { Participant } \\
\text { recruitment } \\
\text { Consecutive } \\
\text { admission from } \\
\text { assessment and } \\
\text { rehabilitation unit } \\
\text { and via postal } \\
\text { questionnaires. } \\
\text { Design } \\
\text { Correlational; } \\
\text { cross-sectional } \\
\text { survey design. } \\
\text { Data analyses } \\
\text { Correlational and } \\
\text { regression } \\
\text { analyses. }\end{array}$ & PTGI & $\begin{array}{l}\text { CPOTS } \\
\text { HADS }\end{array}$ & $\begin{array}{l}\text { Tedeschi \& } \\
\text { Calhoun's } \\
\text { model of PTG } \\
\text { (1995). }\end{array}$ & $\begin{array}{l}\text { 1) Positive relationship between } \\
\text { PTG and } 4 \text { indicators of cognitive } \\
\text { processing. } \\
\text { 2) Negative relationship between } \\
\text { PTG and depression. } \\
\text { 3) Time moderated relationships } \\
\text { between PTG and } \\
\text { anxiety/depression (>negative); } \\
\text { and relationship between PTG and } \\
2 \text { aspects of cognitive processing - } \\
\text { downward comparisons and } \\
\text { resolutions (>positive). }\end{array}$ & $\begin{array}{l}\text { 1) Small sample size. } \\
\text { 2) Limited ethnic } \\
\text { diversity in sample. } \\
\text { 3) Mixed recruitment; } \\
\text { generalisability of } \\
\text { findings unclear. } \\
\text { 4) Cross-sectional } \\
\text { design - direction of } \\
\text { relationships. }\end{array}$ \\
\hline $\begin{array}{l}\text { Gillen } \\
(2005)\end{array}$ & USA & $\begin{array}{l}\mathbf{N}=\mathbf{1 6} \\
\text { Stroke survivors } \\
\text { (Male }=8 ; \\
\text { Female }=8) \\
\frac{\text { Age }}{(\mathrm{M}=61 ;} \\
\mathrm{SD}=11) \\
\frac{\text { Time since }}{\text { Stroke }} \\
\text { Not stated. }\end{array}$ & $\begin{array}{l}\text { Participant } \\
\text { recruitment } \\
\text { Consecutive } \\
\text { admission from } \\
\text { rehabilitation } \\
\text { unit. } \\
\text { Design } \\
\text { Cross-sectional; } \\
\text { Case series using } \\
\text { qualitative } \\
\text { analytic }\end{array}$ & $\begin{array}{l}\text { Themes } \\
\text { relating to } \\
\text { PTG were } \\
\text { identified. }\end{array}$ & $\begin{array}{l}\text { None } \\
\text { stated. }\end{array}$ & None used. & $\begin{array}{l}\text { 10/16 identified positive } \\
\text { consequences of stroke. } \\
\text { Themes identified: } \\
\text { 1) Increased social relationships } \\
\text { 2) Increased health awareness } \\
\text { 3) Change in religious life } \\
\text { 4) Personal growth, } \\
\text { 5) Altruism }\end{array}$ & $\begin{array}{l}\text { 1) Small sample size } \\
\text { 2) PTG was not } \\
\text { assessed with a } \\
\text { standardised measure. } \\
\text { 3) Did not focus on any } \\
\text { correlates of PTG after } \\
\text { stroke. }\end{array}$ \\
\hline
\end{tabular}




\begin{tabular}{|c|c|c|c|c|c|c|c|c|}
\hline & & & $\begin{array}{l}\text { Techniques; } \\
\text { standardised } \\
\text { open-ended } \\
\text { questions. } \\
\text { Data analyses } \\
\text { Frequency } \\
\text { distributions of } \\
\text { the response set; } \\
\text { Software } \\
\text { (NUD*IST v5) } \\
\text { used to analyse } \\
\text { themes. } \\
\text { Pattern matching } \\
\text { techniques (Yin, } \\
\text { 2003) }\end{array}$ & & & & & \\
\hline $\begin{array}{l}\text { Kuenemund } \\
\text { et al. } \\
(2014)\end{array}$ & Germany & 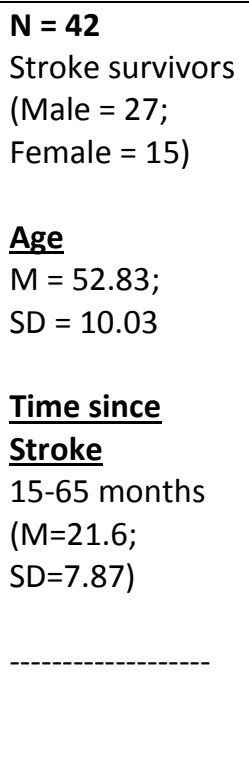 & $\begin{array}{l}\text { Participant } \\
\frac{\text { recruitment }}{\text { Convenience }} \\
\text { sampling. } \\
\underline{\text { Design }} \\
\text { Mixed design; } \\
\text { case-control; } \\
\text { cross-sectional; } \\
\text { qualitative (semi- } \\
\text { structured } \\
\text { Interview). } \\
\text { Data analyses } \\
\text { ANOVA; } \\
\text { MANCOVA; } \\
\text { Qualitative data } \\
\text { coded. }\end{array}$ & $\begin{array}{l}\text { PTGI (GV) } \\
\text { Codes } \\
\text { relating to } \\
\text { PTG were } \\
\text { identified }\end{array}$ & $\begin{array}{l}\text { ADFIQ } \\
\text { SWLS } \\
\text { COES } \\
\text { CES-D } \\
\text { (GV) }\end{array}$ & $\begin{array}{l}\text { Tedeschi \& } \\
\text { Calhoun's } \\
\text { model of PTG } \\
\text { (1995). } \\
\text { Meaning } \\
\text { maintenance } \\
\text { model (Heine } \\
\text { et al. 2006). }\end{array}$ & $\begin{array}{l}\text { Quantitative Findings } \\
\text { 1) Stroke survivors showed } \\
\text { significantly higher PTG than } \\
\text { controls }(F(1,75)=9.79, P=.003) \text {. } \\
\text { 2) Stroke survivors integrated the } \\
\text { traumatic event into their identity } \\
\text { more than controls }(F(1,74)= \\
\text { 37.54, P<.001) } \\
\text { 3) Stroke survivors had significantly } \\
\text { higher depression score and lower } \\
\text { satisfaction with life scores relative } \\
\text { to controls. }\end{array}$ & $\begin{array}{l}\text { 1) Sample size small - } \\
\text { generalizability of } \\
\text { results limited. } \\
\text { 2) High rate of attrition } \\
\text { at follow-up sample. } \\
\text { 3) Cross-sectional } \\
\text { design. } \\
\text { 4) Difference in time } \\
\text { since event - not } \\
\text { matched. }\end{array}$ \\
\hline
\end{tabular}




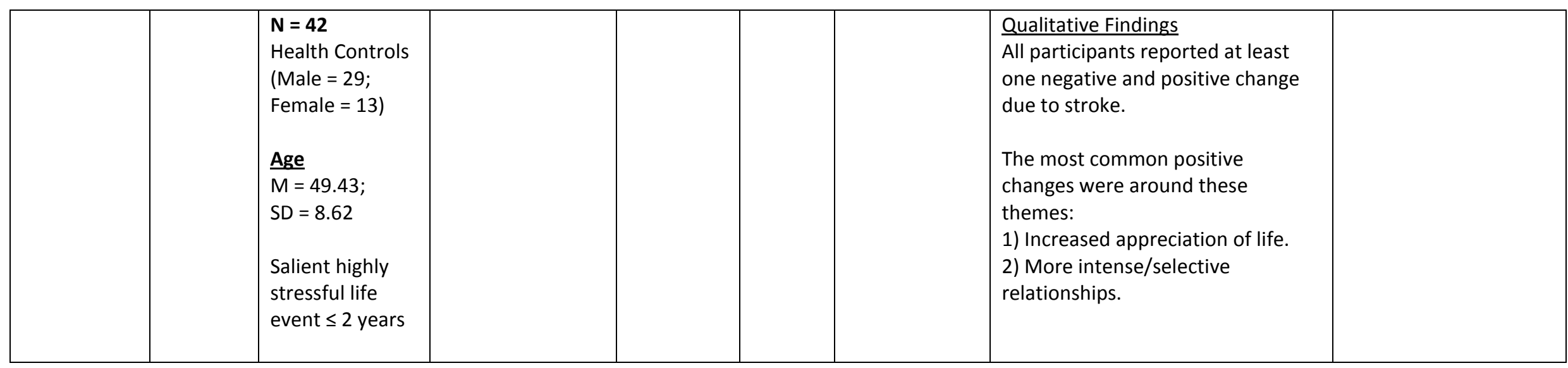

M = Mean; SD = Standard Deviation; PTGI = Posttraumatic Growth Inventory (Tedeschi \& Calhoun, 1996); CPOTS = Cognitive Processing of Trauma Scale (Williams et al. 2002); HADS = Hospital Anxiety and Depression Scale (Zigmond \& Snaith, 1983); PTGI (GV) = Posttraumatic Growth Inventory (German Version) (Maercker \& Langner, 2001); ADFIQ = Aachen Daily-Functioning Item Bank Questionnaire (Böcker et al. 2009); SWLS = Satisfaction with Life Scale (Diener et al. 1985); COES = Centrality of Event Scale (Berntsen \& Rubin, 2006); CES-D (GV) = Center for Epidemiological Studies Depression scale abbreviated form (German Version) (Hautzinger \& Bailer, 1993); UK = United Kingdom; USA = United States of America 
Table 1.2 Evaluation of correlational/cross sectional studies using the SURE Quality Framework for the initial review.

\begin{tabular}{|c|c|c|}
\hline Quality Framework criteria & Gangstad et al. (2009) & Kuenemund et al. (2014) \\
\hline \multicolumn{3}{|l|}{ Section 1: Population } \\
\hline \multirow[t]{2}{*}{ Is the source population or source area well described? } & $\begin{array}{l}\text { Country, setting and demographics } \\
\text { reported. }\end{array}$ & Country, setting and demographics reported. \\
\hline & 2 & 2 \\
\hline \multirow[t]{2}{*}{ Is the eligible population of area representative of the source population or area? } & Consecutive sampling; NHS patients. & Convenience sampling; hospital patients. \\
\hline & 2 & 1 \\
\hline \multirow[t]{2}{*}{ Do the selected participants or areas represent the eligible population or area? } & $\begin{array}{l}\text { Sampling, inclusion/exclusion criteria and } \\
\text { response rate reported }\end{array}$ & $\begin{array}{l}\text { Sampling, inclusion/exclusion criteria and } \\
\text { response rate reported }\end{array}$ \\
\hline & 2 & 2 \\
\hline \multicolumn{3}{|l|}{ Section 2: Method of selection of exposure (or comparison) group } \\
\hline \multirow[t]{2}{*}{ Selection of exposure (and comparison) group. How was selection bias minimised? } & Consecutive $\&$ convenience sampling. & $\begin{array}{l}\text { Convenience. Healthy controls using snowball } \\
\text { sampling. }\end{array}$ \\
\hline & 1 & 1 \\
\hline \multirow[t]{2}{*}{ Was the selection of explanatory variables based on a sound theoretical basis? } & Hypotheses based on model of PTG. & Hypotheses based on model of PTG. \\
\hline & 2 & 2 \\
\hline \multirow[t]{2}{*}{ Was the contamination acceptably low? } & Not applicable & Asked to name salient, stressful life event. \\
\hline & 0 & 1 \\
\hline \multirow[t]{2}{*}{ How well were likely confounding factors identified and controlled? } & $\begin{array}{l}\text { Attempt to control for confounding factors } \\
\text { in design }\end{array}$ & $\begin{array}{l}\text { Design, control group. Adjustments were } \\
\text { made for confounding effects. }\end{array}$ \\
\hline & 1 & 2 \\
\hline \multirow[t]{2}{*}{ Is the setting applicable to the UK? } & UK sample & Not reported. Unknown. \\
\hline & 2 & $\mathbf{0}$ \\
\hline \multicolumn{3}{|l|}{ Section 3: Outcomes } \\
\hline \multirow[t]{2}{*}{ Were the outcome measures and procedures reliable? } & $\begin{array}{c}\text { Internal reliability reported and evaluated } \\
\text { using comparable data. }\end{array}$ & Reliability demonstrated and reported. \\
\hline & 2 & 2 \\
\hline \multirow[t]{2}{*}{ Were the outcome measurements complete? } & 45 completed questionnaires returned. & 42 completed questionnaires returned. \\
\hline & 2 & 2 \\
\hline \multirow[t]{2}{*}{ Were all the important outcomes assessed? } & PTG, mood and cognitive processing. & PTG, mood, functioning and event centrality. \\
\hline & 2 & 2 \\
\hline
\end{tabular}




\begin{tabular}{|c|c|c|}
\hline \multirow[t]{2}{*}{ Was there a similar follow-up time in exposure and comparison groups? } & Not applicable & Not applicable \\
\hline & 0 & 0 \\
\hline \multirow[t]{2}{*}{ Was follow-up time meaningful? } & Not applicable & Not applicable \\
\hline & $\mathbf{0}$ & $\mathbf{0}$ \\
\hline \multicolumn{3}{|l|}{ Section 4: Analyses } \\
\hline \multirow[t]{2}{*}{ Was the study sufficiently powered to detect an intervention effect (if one exists)? } & Not reported & Not reported \\
\hline & 0 & 0 \\
\hline \multirow[t]{2}{*}{ Were multiple explanatory variables considered in the analyses? } & $\begin{array}{l}\text { Model used to consider explanatory } \\
\text { variables. }\end{array}$ & Model used to consider explanatory variables. \\
\hline & 2 & 2 \\
\hline \multirow[t]{2}{*}{ Were the analytical methods appropriate? } & Inferential statistics appropriate. & Mixed methods appropriate. \\
\hline & 2 & 2 \\
\hline \multirow[t]{2}{*}{ Was the precision of association given or calculable? Is association meaningful? } & Confidence intervals and $\mathrm{p}$ values reported. & $P$ values reported. \\
\hline & 2 & 1 \\
\hline \multicolumn{3}{|l|}{ Section 5: Summary } \\
\hline \multirow[t]{2}{*}{ Are the study results internally valid (i.e. unbiased)? } & $\begin{array}{l}\text { Good reliability. Unknown whether } \\
\text { potential confounding variables were } \\
\text { adjusted in analysis. Potential for bias. }\end{array}$ & $\begin{array}{l}\text { Good reliability. Confounding factors } \\
\text { managed but potential for bias. }\end{array}$ \\
\hline & 1 & 1 \\
\hline \multirow[t]{2}{*}{ Are the findings generalisable to the source population (i.e. externally valid)? } & $\begin{array}{c}\text { Mixed sampling, source and eligible } \\
\text { population reported. }\end{array}$ & $\begin{array}{c}\text { Convenience sampling, use of control group, } \\
\text { source and eligible population reported. }\end{array}$ \\
\hline & 1 & 2 \\
\hline $\begin{array}{l}\text { Total Quality Score: } \\
\text { External Validity (EV) Score } \\
\text { Internal Validity (IV) Score }\end{array}$ & $\begin{array}{l}26 / 34 \\
6 / 6 \\
20 / 28\end{array}$ & $\begin{array}{l}25 / 34 \\
5 / 6 \\
20 / 28\end{array}$ \\
\hline
\end{tabular}


Table 1.3 Evaluation of the qualitative study using the SURE Quality framework for the initial review.

\begin{tabular}{|c|c|}
\hline & Scoring: Yes, Can't tell, or No. \\
\hline & Gillen (2005) \\
\hline $\begin{array}{l}\text { Does the study address a clearly focused question/hypothesis? } \\
\text { Setting? } \\
\text { Perspective? } \\
\text { Intervention or Phenomena Comparator/control (if any)? } \\
\text { Evaluation/Exploration? }\end{array}$ & $\begin{array}{l}\text { Yes } \\
\text { Yes } \\
\text { Yes } \\
\text { N/A } \\
\text { Yes }\end{array}$ \\
\hline $\begin{array}{l}\text { Is the choice of qualitative method appropriate? } \\
\text { Is it an exploration of eg behaviour/reasoning/ beliefs)? } \\
\text { Do the authors discuss how they decided which method to use? }\end{array}$ & $\begin{array}{l}\text { Yes } \\
\text { Yes } \\
\text { No }\end{array}$ \\
\hline $\begin{array}{l}\text { Is the sampling strategy clearly described and justified? Is the sampling strategy clearly described and justified? } \\
\text { Is it clear how participants were selected? } \\
\text { Do the authors explain why they selected these particular participants? } \\
\text { Is detailed information provided about participant characteristics and about those who chose not to participate? }\end{array}$ & $\begin{array}{l}\text { Yes } \\
\text { Yes } \\
\text { Yes } \\
\text { No }\end{array}$ \\
\hline $\begin{array}{l}\text { Is the method of data collection well described? } \\
\text { Was the setting appropriate for data collection? } \\
\text { Is it clear what methods were used to collect data? } \\
\text { Type of method (eg, focus groups, interviews, open questionnaire etc) and tools (eg notes, audio, audio visual recording). } \\
\text { Is there sufficient detail of the methods used (eg how any topics/questions were generated and whether they were piloted; if } \\
\text { observation was used, whether the context described and were observations made in a variety of circumstances? } \\
\text { Were the methods modified during the study? If YES, is this explained? } \\
\text { Is there triangulation of data (ie more than one source of data collection)? } \\
\text { Do the authors report achieving data saturation? }\end{array}$ & $\begin{array}{l}\text { Yes } \\
\text { Can't tell } \\
\text { Yes } \\
\text { (Interview script; } \\
\text { notes) } \\
\text { Can't tell } \\
\text { No } \\
\text { No }\end{array}$ \\
\hline $\begin{array}{l}\text { Is the relationship between the researcher(s) and participants explored? } \\
\text { Did the researcher report critically examining/reflecting on their role and any relationship with participants particularly in relation to } \\
\text { formulating research questions and collecting data). } \\
\text { Were any potential power relationships involved (ie relationships that could influence in the way in which participants respond)? }\end{array}$ & $\begin{array}{l}\text { No } \\
\text { No } \\
\text { No }\end{array}$ \\
\hline
\end{tabular}


Are ethical issues explicitly discussed?

Is there sufficient information on how the research was explained to participants?

Was ethical approval sought?

Are there any potential confidentiality issues in relation to data collection?

Is the data analysis/interpretation process described and justified?

Is it clear how the themes and concepts were identified in the data?

Can't tell

Was the analysis was performed by more than one researcher?

No

Yes

Yes

are negative/discrepant results taken into account?

\section{Are the findings credible?}

Are there sufficient data to support the findings?

Are sequences from the original data presented (eg quotations) and were these fairly selected?

Are the data rich (ie are the participants' voices foregrounded)?

Are the explanations for the results plausible and coherent?

Are the results of the study compared with those from other studies?

\section{Is any sponsorship/conflict of interest reported?}

\section{Finally...consider:}

Did the authors identify any limitations?

Are the conclusions the same in the abstract and the full text? 


\subsubsection{Samples and populations}

Qualitative studies have yielded findings in support of the prevalence of PTG in stroke survivors. Gillen (2005) found that $63 \%$ of participants $(N=16)$ experienced positive consequences after stroke. Positive consequences included: increased social relationships, increased health awareness, change in religious life, personal growth and altruism. Kuenemund et al. (2014) found that all participants reported at least one positive and one negative change due to stroke $(\mathrm{N}=26)$. The most common negative changes reported included the categories of 'physical impairments' and 'restriction in daily life', whereas the most common positive changes included 'increased appreciation of life', 'higher appreciation of relationships' and 'more selective and closer relationships'. Of note, the category of 'increased awareness of limited life time' was rated as neutral, neither a positive or negative change. Elsewhere in the literature, awareness of one's mortality is associated with lower rates of PTG when reminded of a recent life-threatening event (Luszczynska et al. 2012)

The prevalence of PTG in stroke survivors has also been demonstrated using quantitative studies (Gangstad et al. 2009; Kuenemund et al. 2014). Of note, the mean PTGI scores reported in these studies (Gangstad et al. 2009: $M=50.93, S D=19.92$; Kuenemund et al. 2014: $M=57.69, S D=19.28$ ) are lower than those reported in previous studies examining PTG after traumatic events such as amputation (Phelps et al. 2008: $M=61.7, S D=24.4$ ) and cancer (Tallman et al. 2010: $M=74.2, S D=18.1$ ). However, the samples of stroke survivors studied have been considerably older (e.g., Gangstad et al. 2009: $M=71.67$, SD = 10.64) than studies examining PTG in other populations. More recently, Kuenemund et al. (2014) found that stroke survivors showed significantly higher PTG compared to healthy controls $(F(1,75)=$ 9.79, $p=.003$ ) wherein the PTGI subscales of 'appreciation of life' and 'relating to others' scored highest for stroke survivors. However, they also found that besides PTG, stroke survivors showed significantly higher depression $(F(1,73)=8.77, p=.004)$ and scored lower satisfaction with life $(F(1,72)=17.58, p \leq .001)$ compared to healthy controls. None of the studies used the PTGI measure longitudinally to assess the development of growth over time.

Kuenemund et al. (2014) screened for inclusion and exclusion criteria using a neuropsychologist who identified whether stroke survivors had adequate language ability and self-awareness to complete self-report questionnaires. Gillen (2005) screened medical 
records for language and cognitive functioning, only inviting participants who scored above six on the Functional Independence Measure TM. Gangstad et al. (2009) report that stroke survivors attending a rehabilitation centre who were unable to complete the questionnaire due to cognitive impairment following the stroke were not invited to participate. However, they do not specify how participants during the second stage of recruitment were screened. It is possible that the $26.5 \%$ response rate to their postal questionnaires could be partly attributable to a lack of screening. Kuenemund et al. (2014) excluded stroke survivors with co-morbid progressive neurodegenerative disease, history of severe mental health difficulties and history of substance misuse. Although excluding participants on the basis of co-morbid diagnoses or cognitive difficulties may minimise potential bias, it also reduces the generalisability of the findings given that cognitive difficulties are common amongst stroke survivors (Adamson et al. 2004).

All of the studies reviewed focused on adult populations with stroke with the mean age of participants ranging from 52.83 to 71.67. Mixed gender samples were used in all of the studies with the mean percentage of female participants ranging from $36 \%$ to $50 \%$ and the mean percentage of male participants ranging from $50 \%$ to $64 \%$. The studies reviewed recruited from a diverse range of populations in different countries (i.e. Gangstad et al. 2009: White British in the United Kingdom; Gillen, 2005: Caucasian = 7, African-American = 5, Hispanic $=4$ in the USA; Kuenemund et al. 2014: native German speakers) which reflect the clinical reality of stroke being a world health issue (WHO, 2012) but limits the generalisability of the findings. The 'healthy controls' used in Kuenemund et al. (2014) were asked to name 'a salient, highly stressful life event that happened within the last two years'. Of note, the time since event for controls $(M=14.67$ months, $S D=7.62)$ was much lower than for stroke survivors $(M=21.6$, $S D=7.87)$.

\subsubsection{Design and recruitment}

Two of the studies employed a cross-sectional design (Kuenemund et al. 2014; Gangstad et al. 2009). Kuenemund et al. (2014) used a mixed methodology to explore identity changes following stroke: case-control methodology and semi-structured interviews. Both studies focused on the relationship between PTG and depression following stroke, albeit using different versions of the PTGI (i.e. Kuenemund et al. 2014 used the German version of the 
PTGI (Maercker \& Langner, 2001)). The reliance on cross-sectional design in these studies limits the possibility of making any inferences regarding the causal relationship between PTG and stroke. The third study employed a case series design using qualitative techniques to explore the positive consequences of stroke in stroke survivors (Gillen, 2005). The strengths of this study are consecutive recruitment of participants within a specific timeframe, a welldefined study protocol, a clear research question, as well as the use of explicit inclusion and exclusion criteria which is in line with guidelines for review (Chan \& Bhandari, 2011).

All studies at least partly recruited from clinical settings. Gillen (2005) recruited participants from a general inpatient rehabilitation unit, Kuenemund et al. (2014) recruited participants through a related ongoing study where participants had originally been recruited through an acute rehabilitation setting. Gangstad et al. (2009) recruited from an outpatient assessment and rehabilitation centre $(\mathrm{N}=15)$ but also recruited participants through sending questionnaires to patients who had attended the centre in the past four years $(N=45)$. Interestingly, there was a low response rate of $26.5 \%$ but Gangstad et al. (2009) found no difference in response to psychological variables between the two recruitment strategies. Gillen (2005) reported that 52 stroke survivors were excluded, whereas Kuenemund et al. (2014) report that of the sample, 11 participants refused consent, 30 participants were not contactable and 1 had died.

Only one study used consecutive sampling (Gillen, 2005), despite another attempting to sample using this technique (Gangstad et al. 2009). Two studies used convenience sampling, Kuenemund et al. (2014) recruited from an existing sample of participants, while Gangstad et al. (2009) recruited the remaining participants through postal survey. However, the healthy controls in Kuenemund et al. (2014) were recruited using a snowball sampling strategy which may have introduced a cluster bias. The studies which employed convenience sampling failed to comment on the generalisability of the finding to the broader population.

\subsubsection{Sample size}

The sample sizes used in the studies reviewed varied considerably (see Table 1.1). Due to the small sample size used in one study (Kuenemund et al. 2014), bootstrapping procedures (Efron \& Tibshirani, 1993) were used to estimate robust parameters for statistical analysis. 
The inconsistent findings between the few studies reviewed regarding the relationship between PTG and depression after stroke may be partly attributable to low power. None of the studies reviewed reported statistical power calculations.

\subsubsection{Treatment of confounding variables}

All of the studies attempted to control for the confounding effects of cognitive impairment and communication difficulties either through screening by a neuropsychologist (Kuenemund et al. 2014) or adherence to the inclusion and exclusion criteria (Gangstad et al. 2009; Gillen, 2005). One study used age-, education- and sex- matched healthy controls (Kuenemund et al. 2009). Several socio-demographic variables (i.e. age, gender and education), as well as time since stroke, were accounted for statistically using multivariate analysis (Gangstad et al. 2009). However, recruiting participants in acute stages following trauma may have confounded associations between PTG and related factors. Aldwin and Levenson (2004) suggest that PTG in the acute phase post-trauma may be illusory and may reflect attempts at coping. None of the studies reported whether participants were taking medications or whether participants had experienced previous traumatic events. Similarly, it is unclear how variables linked to personality were accounted for (e.g. self-confidence, resilience optimism, self-efficacy, motivation).

\subsubsection{Review of the study findings}

All of the studies reviewed demonstrated evidence of PTG in stroke survivors (Gillen, 2005; Gangstad et al. 2009; Kuenemund et al. 2014). Gangstad et al. (2009) found that rates of PTG in stroke survivors were lower than for survivors of other health related difficulties (e.g. cancer; Cordova et al. 2001). One of the discernible differences between studies of stroke survivors and cancer survivors is that studies of stroke survivors have generally had a higher mean age than cancer survivors (Gangstad et al. 2009). However, Kuenemund et al. (2014) has recently found evidence of PTG in stroke survivors with a much lower mean age than that reported by Gangstad et al. (2009) which suggests that PTG can occur for stroke survivors across a wide age-range. Furthermore, Kuenemund et al. (2014) found that stroke survivors experienced significantly more PTG than healthy age-matched controls. 
In terms of the question underpinning this systematic review, these studies provide some good quality findings, albeit limited in nature, regarding the predictors of PTG in stroke survivors. Using a correlational design, Gangstad et al. (2009) found that PTG in stroke survivors was positively associated with four aspects of cognitive processing which included: positive cognitive restructuring, downward comparison, resolution and denial. Of interest, they also found that as time since stroke increased, the relationship between PTG and two aspects of cognitive processing (i.e. downward comparisons and resolution) became more positive and significant. Such findings point toward the role of rumination in PTG but do not provide evidence for the role of rumination in mediating the effect of psychological distress on PTG. Also noteworthy is a study (Hallam \& Morris, 2014), external to this review, which found that rumination mediated the effect of social support on PTG in stroke carers. The aforementioned study also found that deliberate rumination accounted for most of the variance in explaining PTG and that rumination together with social support, acceptance coping and survivor functioning accounted for most of the variance in predicting PTG. This pattern of findings provides support for Tedeschi and Calhoun's model of PTG $(1995,2004)$. However, as the findings of this review demonstrate, similar studies have not been conducted using stroke survivors.

Gangstad et al. (2009) found that PTG was negatively associated with depression and notably, as time since stroke increased, the association between PTG and depression became more negative and significant. In contrast, Kuenemund et al. (2014) found that stroke survivors reported significantly higher depression scores and lower satisfaction with life compared to controls. Using a semi-structured interview, Kuenemund et al. (2014) found evidence of the negative changes which can co-exist alongside positive changes. This provides support for the theory that changes following stroke need to be appraised using dialectical thinking in order for PTG to occur (Tedeschi \& Calhoun, 2004). Kuenemund et al. (2014) found that the most common negative changes perceived following stroke included factors relating to physical impairment and restriction in daily life. This finding is consistent with what is known about the complex disability which can follow stroke and points toward the role of disability in the perception of negative changes after stroke (Adamson et al. 2004). 
Collectively, these findings suggest that mood varies over the course of PTG development and this supports the theory that an experience of 'crisis' is integral to cognitive processing necessary for PTG to develop (Tedeschi \& Calhoun, 2004). Kuenemund et al. (2004) found that stroke survivors show significantly higher event centrality when compared to controls which suggests that stroke survivors redefine their sense of self post-stroke, to a degree which exceeds the normal trajectory of development. This supports the theory that the transformative changes associated with PTG go beyond pre-trauma levels of psychological functioning.

In summary, there is evidence to suggest that aspects of cognitive processing or rumination may predict PTG (Gangstad et al. 2009). The findings reported regarding the role of mood related difficulties in predicting PTG support the complex nature of emotional processing following stroke. One study found that depression reduced over time (Gangstad et al. 2009) whereas another study found that stroke survivors reported significantly more depression and lower quality of life than controls (Kuenemund et al. 2014). Such findings evidence the range of psychological experiences stroke survivors may have followingstroke but highlight how little is known regarding the possible trajectory of psychological experience post-stroke. One study found that stroke survivors showed significantly higher event centrality post-stroke compared to controls which supports the re-appraisal of identity characteristic of PTG. Kuenemund et al. (2014) have demonstrated that positive changes can co-exist alongside negative changes such as factors relating to physical impairment and physical restriction in daily life which supports models of PTG (e.g. Tedeschi \& Calhoun, 1995, 2004). However, they did not provide evidence of a direct association between daily functioning and PTG.

One of the major limitations of the literature on PTG in stroke survivors, as identified in this review, is that there are no known studies which have found evidence in support of the mechanisms of individual change integral to models of PTG (e.g. Tedeschi \& Calhoun, 2004) in stroke survivors. Of note, one study (i.e. Hallam \& Morris, 2014), external to this review, has found evidence in support of the mechanism change proposed by Tedeschi and Calhoun (2004) (i.e. rumination mediated the effect of social support on PTG). Additionally, Hallam and Morris (2014) found that rumination, social support, acceptance coping and daily 
functioning were most predictive of PTG in stroke carers, but this finding has not been replicated using stroke survivors.

Despite the aforementioned strengths and shortcoming of the studies discussed, a range of other factors impacted on the overall quality of the findings presented. For example, all of the studies reviewed used different measures of PTG and were conducted in different countries which impacts on the generalisability of the findings. Although all of the studies excluded participants on the basis of cognitive and communication difficulties in an effort to reduce the role of confounding factors, this most likely resulted in the experiences of stroke survivors who experience significant communication difficulties not being sampled. Furthermore, the reliance on using a cross-sectional methodology in these studies limits the causal inferences which could be made between PTG and stroke, as well as our understanding of the developmental trajectory of PTG.

\subsubsection{Expanded systematic review}

\subsubsection{Review Methodology}

Given the paucity of research on posttraumatic growth in stroke survivors, a further systematic literature search was carried out to explore posttraumatic growth in physical health conditions other than stroke. The World Health Organisation (2012) lists ischaemic heart disease as the leading cause of death in the world. Given that ischaemic heart disease is the only condition listed as more life-threatening than stroke, it could be considered as a traumatic stressor (DSM-IV, 2000) potentiating PTG. Furthermore, myocardial infarction (MI), or a heart attack, is a traumatic event associated with ischaemic heart disease and a comparable traumatic event given that stroke is often described as a brain attack (Stroke Association, 2015). This systematic review was conducted in order to answer the following question: "What are the predictors of posttraumatic growth in survivors of myocardial infarction?"

On the $19^{\text {th }}$ of January 2014, a review of the clinical research evidence was conducted using the following databases: EMBASE (1947-present), Ovid MEDLINE (R) (1946-present), PsycINFO (1806-present) and PsycArticles Full Text. The search terms focused on two key 
areas: 'posttraumatic growth' and 'ischaemic heart disease' (see Appendix B for a complete list of the search terms used). The search terms were combined using Boolean operators (i.e. 'and', 'or'). All abstracts and titles identified during this search were reviewed ( $N=190$ after removal of duplicates).

Studies were selected for further review if participants had a myocardial infarction and if posttraumatic growth was measured either quantitatively or qualitatively. Only peerreviewed, original articles, published in English between 1980 and 2014 were reviewed. Studies which included participants younger than 18 years of age and review or discussion papers were excluded.

A flow chart illustrating the selection process for studies included in the systematic review can be found in Figure 1.4 .

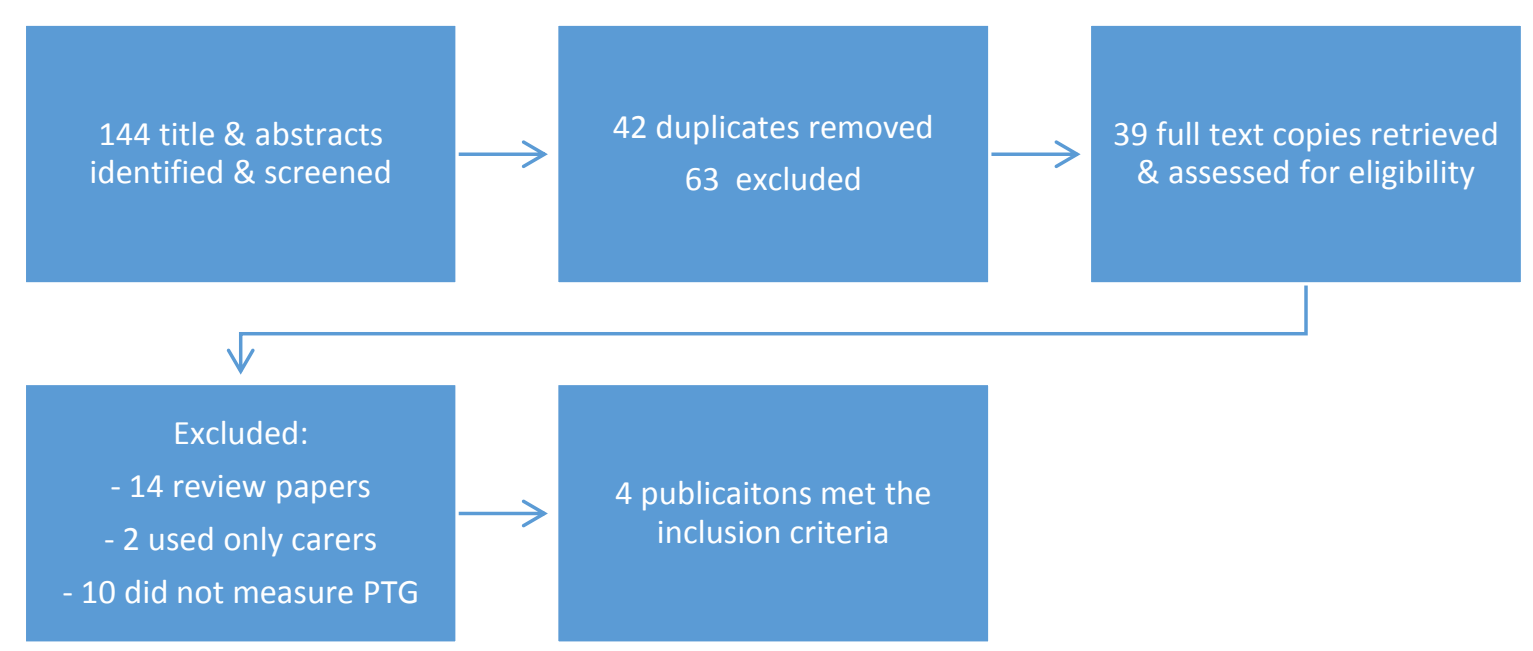

Figure 1.4 Flow chart of the procedure for selecting studies for expanded systematic review

\subsubsection{Review results and quality framework}

An overview of the four studies which met the inclusion criteria for further review can be found in Table 1.4. The Support Unit for Research Evidence (SURE) frameworks for cross sectional/correlational studies and qualitative studies were used to evaluate the studies which met the inclusion criteria for review (see Table 1.5 and Table 1.6). For information on 
scoring, see section 1.5.2.2. A narrative review which examines the studies in light of the review question as other aspects of quality such as sampling, design, participant recruitment, treatment of confounding factors is also presented below. 
Table 1.4 Summary of the studies which met the inclusion criteria for the expanded systematic review.

\begin{tabular}{|c|c|c|c|c|c|c|c|c|}
\hline Paper & Country & Sample & Method & $\begin{array}{c}\text { Measure } \\
\text { of PTG }\end{array}$ & $\begin{array}{c}\text { Other } \\
\text { Measures }\end{array}$ & $\begin{array}{c}\text { Theoretical } \\
\text { Model }\end{array}$ & Key Findings & Key Limitations \\
\hline $\begin{array}{l}\text { Bluvstein et al. } \\
\text { (2012) }\end{array}$ & Israel & 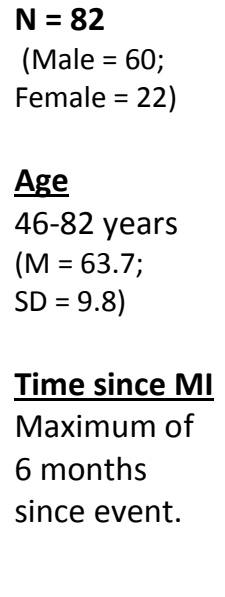 & $\begin{array}{l}\frac{\text { Participant }}{\text { recruitment }} \\
\begin{array}{l}\text { Consecutive } \\
\text { admission. }\end{array} \\
\frac{\text { Design }}{\text { Correlational; cross- }} \\
\text { sectional survey } \\
\text { design. } \\
\text { Data analyses } \\
\text { Correlational and } \\
\text { moderational } \\
\text { analyses. }\end{array}$ & PTGI & $\begin{array}{l}\text { PTSD } \\
\text { inventory } \\
\text { HRQOL }\end{array}$ & $\begin{array}{l}\text { Tedeschi \& } \\
\text { Calhoun's } \\
\text { model of PTG } \\
(1995)\end{array}$ & $\begin{array}{l}\text { 1) } 17.1 \% \text { had significant } \\
\text { PTSS, } 71.2 \% \text { reported PTG. } \\
\text { 2) PTSS positively related } \\
\text { to PTG and psychological } \\
\text { distress. } \\
\text { 3) PTSS negatively related } \\
\text { to well-being and HRQOL. } \\
\text { 4) PTG moderated the } \\
\text { relationship between PTSS } \\
\text { and mental health } \\
\text { outcomes. }\end{array}$ & $\begin{array}{l}\text { 1) Timing relative to } \\
\text { the cardiac event - } \\
\text { may explain low leve } \\
\text { of growth. } \\
\text { 2) Bias introduced by } \\
\text { recruiting from } \\
\text { rehabilitation setting } \\
\text { 3) Cross-sectional } \\
\text { study. } \\
\text { 4) Time since event } \\
\text { not documented. } \\
\text { 4) Small sample size. }\end{array}$ \\
\hline $\begin{array}{c}\text { Senol-Durak \& } \\
\text { Ayvasik } \\
(2010)\end{array}$ & Turkey & $\begin{array}{l}\mathbf{N}=\mathbf{1 4 8} \\
\text { MIP } \\
\text { (Male = 129; } \\
\text { Female = 19) } \\
\frac{\text { Age }}{27-80} \\
(\mathrm{M}=56 ; \\
\mathrm{SD}=10.66) \\
\text { Time since MI } \\
\mathrm{M}=1371.7 \\
\text { days; SD = } \\
239.5)\end{array}$ & $\begin{array}{l}\text { Participant } \\
\text { recruitment } \\
\text { Consecutive } \\
\text { admission. } \\
\text { Design } \\
\text { Structural equation } \\
\text { analysis; } \\
\text { correlational; cross- } \\
\text { sectional. } \\
\text { Data analyses } \\
\text { Structural equation } \\
\text { modelling }\end{array}$ & PTGI & $\begin{array}{l}\text { WCQ } \\
\text { MSPSS } \\
\text { DIF }\end{array}$ & $\begin{array}{l}\text { Schaefer \& } \\
\text { Moos' model } \\
\text { of PTG (1998) }\end{array}$ & $\begin{array}{l}\text { 1) PSS positively related to } \\
\text { coping and PE. } \\
\text { 2) Coping was positively } \\
\text { related to PTG. } \\
\text { 3) PE not significantly } \\
\text { related to PTG. } \\
\text { 4) The relationship } \\
\text { between PSS and PTG was } \\
\text { predicted by coping. } \\
\text { 5) PE did not mediate the } \\
\text { relationship between PSS } \\
\text { and PTG. }\end{array}$ & $\begin{array}{l}\text { 1) Small sample size. } \\
\text { 2) Generalisability of } \\
\text { findings }\end{array}$ \\
\hline
\end{tabular}




\begin{tabular}{|c|c|c|c|c|c|c|c|c|}
\hline $\begin{array}{c}\text { Garnefski et al. } \\
\qquad(2008)\end{array}$ & $\begin{array}{l}\text { The } \\
\text { Nether- } \\
\text { lands }\end{array}$ & $\begin{array}{l}\mathbf{N}=139 \\
\text { MIP } \\
\text { (Male = 114; } \\
\text { Female = 25) } \\
\frac{\text { Age }}{\mathrm{M}=56.39 ;} \\
\mathrm{SD}=8.19 \\
\frac{\text { Time since MI }}{3-12 \text { months }} \\
\text { prior to } \\
\text { recruitment }\end{array}$ & 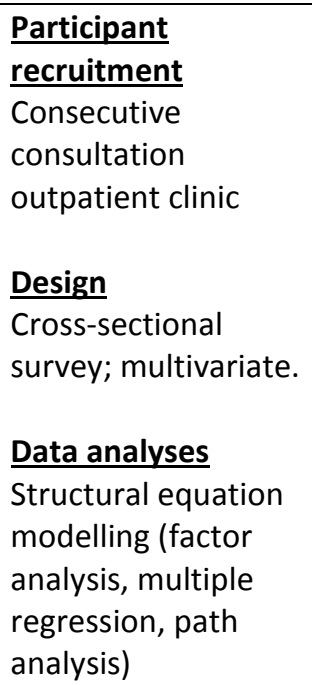 & PGS & $\begin{array}{l}\text { NEO-FFI } \\
\text { HADS } \\
\text { WHO-5 } \\
\text { CERQ }\end{array}$ & None stated. & $\begin{array}{l}\text { 1) Psychological health and } \\
\text { personality predicted PTG } \\
\text { ( } 8 \% \text { and } 18 \% \text { of variance } \\
\text { respectively). } \\
\text { 2) Controlling for gender, } \\
\text { age and post-MI time, the } \\
\text { largest part of the variance } \\
\text { (24\%) of PTG was explained } \\
\text { by cognitive coping } \\
\text { strategies. } \\
\text { 2) The cognitive coping } \\
\text { strategies which predicted } \\
\text { PTG were: putting into } \\
\text { perspective, positive } \\
\text { refocusing and positive } \\
\text { reappraisal. }\end{array}$ & $\begin{array}{l}\text { 1) Use of self- } \\
\text { reported evaluations } \\
\text { for IVs and DVs. } \\
\text { 2) Mental health } \\
\text { before MI unknown. } \\
\text { 3) Anxiety, social } \\
\text { support and goal } \\
\text { adjustment were not } \\
\text { measured. } \\
\text { 4) Cross-sectional } \\
\text { design. } \\
\text { 5) Does not explore } \\
\text { how PTG develops } \\
\text { over time. }\end{array}$ \\
\hline $\begin{array}{l}\text { Panagopoulou } \\
\text { et al. (2009) }\end{array}$ & Greece & $\begin{array}{l}\text { N= 22 } \\
(\mathrm{MIP}=11 ; \\
\text { Partner = 11) } \\
\frac{\text { Age }}{45-70} \\
\text { Time since MI } \\
\text { Unknown. }\end{array}$ & $\begin{array}{l}\text { Participant } \\
\frac{\text { recruitment }}{\text { Consecutive }} \\
\text { admission. } \\
\text { Design } \\
\text { Cross-sectional; } \\
\text { Semi-structured } \\
\text { interview; dyadic } \\
\text { Data analyses } \\
\text { IPA }\end{array}$ & $\begin{array}{l}\text { Themes } \\
\text { relating to } \\
\text { PTG were } \\
\text { identified }\end{array}$ & None. & $\begin{array}{l}\text { Stress and } \\
\text { coping model } \\
\text { (Park \& } \\
\text { Folkman, } \\
\text { 1997) }\end{array}$ & $\begin{array}{l}\text { Themes are suggestive of } \\
\text { benefit finding as } \\
\text { deliberate coping process } \\
\text { for MIP - consistent with } \\
\text { model (Park \& Folkman, } \\
\text { 1997). } \\
\text { Themes identified: } \\
\text { MIP: 1) A catalyst for } \\
\text { change/wake-up call. 2) } \\
\text { Being protected/ invisible. } \\
\text { 3) Focus on others } \\
\text { Partners: 1) Active search } \\
\text { for benefit. } \\
\text { 2) Failure to find benefit. }\end{array}$ & $\begin{array}{l}\text { 1) Findings suggest } \\
\text { limited compatability } \\
\text { of dyadic approach to } \\
\text { event. } \\
\text { 2) Generalisability of } \\
\text { findings to other } \\
\text { contexts. }\end{array}$ \\
\hline
\end{tabular}

MI = myocardial infarction; $\mathbf{M}$ = mean; SD = standard deviation; PTGI = Posttraumatic Growth Inventory (Tedeschi \& Calhoun, 1995); NEO-FFI = Neuroticism Extraversion Openness-Five Factor Invenory (Costa \& McCrae, 1992); HADS = Hospital Anxiety and Depression Scale (Zigmond \& Snaith, 1983); WHO-5 = Wellbeing Questionnaire (Bech, 
1998); CER-Q = Cognitive Emotion Regulation Questionnaire (Garnefski et al. 2002); WCG = Ways of Coping (Folkman \& Lazarus, 1980); MSPSS = Multidimensional Scale of Perceived Social Support (Zimet et al. 1988); DIF = demographic information form. PTSD inventory = Posttraumatic Stress Disorder Inventory (Solomon et al. 1993); HRQOL $=$ Health Related Quality of Life (Muller-Nordhorn et al. 2005). 
Table 1.5 Evaluation of correlational/cross sectional studies using the SURE Quality Framework for the expanded review.

\begin{tabular}{|c|c|c|c|}
\hline Quality Framework criteria & $\begin{array}{l}\text { Bluvstein } \\
\text { et al. (2012) }\end{array}$ & $\begin{array}{l}\text { Garnefski } \\
\text { et al. (2008) }\end{array}$ & $\begin{array}{c}\text { Senol-Durak \& Ayvasik } \\
\text { (2010) }\end{array}$ \\
\hline \multicolumn{4}{|l|}{ Section 1: Population } \\
\hline \multirow[t]{2}{*}{ Is the source population or source area well described? } & $\begin{array}{l}\text { Country, setting and } \\
\text { demographics reported. }\end{array}$ & $\begin{array}{l}\text { Country, setting and } \\
\text { demographics reported. }\end{array}$ & $\begin{array}{c}\text { Country, setting and } \\
\text { demographics reported. }\end{array}$ \\
\hline & 2 & 2 & 2 \\
\hline \multirow[t]{2}{*}{ Is the eligible population of area representative of the source population or area? } & $\begin{array}{l}\text { Consecutive sampling; } \\
\text { hospital patients. }\end{array}$ & $\begin{array}{l}\text { Convenience sampling; } \\
\text { hospital patients. }\end{array}$ & $\begin{array}{l}\text { Convenience sampling; } \\
\text { hospital patients. }\end{array}$ \\
\hline & $\mathbf{2}$ & 2 & \\
\hline \multirow[t]{2}{*}{ Do the selected participants or areas represent the eligible population or area? } & $\begin{array}{l}\text { Sampling and response rate } \\
\text { reported. Unclear } \\
\text { inclusion/exclusion criteria }\end{array}$ & $\begin{array}{l}\text { Sampling, } \\
\text { inclusion/exclusion criteria } \\
\text { and response rate reported }\end{array}$ & $\begin{array}{l}\text { Sampling, } \\
\text { inclusion/exclusion criteria } \\
\text { and response rate reported }\end{array}$ \\
\hline & 1 & 2 & 2 \\
\hline \multicolumn{4}{|l|}{ Section 2: Method of selection of exposure (or comparison) group } \\
\hline \multirow[t]{2}{*}{ Selection of exposure (and comparison) group. How was selection bias minimised? } & Consecutive sampling. & Consecutive sampling. & Consecutive sampling. \\
\hline & 2 & 2 & 2 \\
\hline \multirow[t]{2}{*}{ Was the selection of explanatory variables based on a sound theoretical basis? } & Based on model of PTG & None reported & Based on model of PTG \\
\hline & $\mathbf{2}$ & 2 & $\mathbf{0}$ \\
\hline \multirow[t]{2}{*}{ Was the contamination acceptably low? } & Not applicable & Not applicable & Not applicable \\
\hline & $\mathbf{0}$ & 0 & 0 \\
\hline \multirow[t]{2}{*}{ How well were likely confounding factors identified and controlled? } & $\begin{array}{l}\text { Controlled for confounding } \\
\text { factors in design }\end{array}$ & $\begin{array}{l}\text { Controlled for confounding } \\
\text { factors in design }\end{array}$ & $\begin{array}{l}\text { Controlled for confounding } \\
\text { factors in design }\end{array}$ \\
\hline & 1 & 1 & 1 \\
\hline \multirow[t]{2}{*}{ Is the setting applicable to the UK? } & Not reported & Not reported & Not reported. \\
\hline & 0 & $\mathbf{0}$ & $\mathbf{0}$ \\
\hline \multicolumn{4}{|l|}{ Section 3: Outcomes } \\
\hline \multirow[t]{2}{*}{ Were the outcome measures and procedures reliable? } & $\begin{array}{c}\text { Internal reliability reported } \\
\text { and evaluated using } \\
\text { comparable data. }\end{array}$ & $\begin{array}{l}\text { Internal reliability of scales } \\
\text { reported. }\end{array}$ & $\begin{array}{l}\text { Internal reliability of scales } \\
\text { reported. }\end{array}$ \\
\hline & 2 & 2 & 2 \\
\hline
\end{tabular}




\begin{tabular}{|c|c|c|c|}
\hline \multirow[t]{2}{*}{ Were the outcome measurements complete? } & Not reported & Not reported. & $\begin{array}{l}148 \text { questionnaires } \\
\text { completed. }\end{array}$ \\
\hline & 0 & 0 & 2 \\
\hline \multirow[t]{2}{*}{ Were all the important outcomes assessed? } & $\begin{array}{l}\text { PTG, PTSS, mental health } \\
\text { and health related QoL }\end{array}$ & $\begin{array}{l}\text { PTG, health, personality } \\
\text { and cognitive coping. }\end{array}$ & $\begin{array}{l}\text { PTG, coping and perceived } \\
\text { social support. }\end{array}$ \\
\hline & 2 & 2 & 2 \\
\hline \multirow[t]{2}{*}{ Was there a similar follow-up time in exposure and comparison groups? } & Not applicable & Not applicable & Not applicable \\
\hline & 0 & 0 & 0 \\
\hline \multirow[t]{2}{*}{ Was follow-up time meaningful? } & Not applicable & Not applicable & Not applicable \\
\hline & 0 & 0 & 0 \\
\hline \multicolumn{4}{|l|}{ Section 4: Analyses } \\
\hline \multirow[t]{2}{*}{ Was the study sufficiently powered to detect an intervention effect (if one exists)? } & Not reported & Not reported & 0 \\
\hline & $\mathbf{0}$ & $\mathbf{0}$ & \\
\hline \multirow[t]{2}{*}{ Were multiple explanatory variables considered in the analyses? } & $\begin{array}{l}\text { Model used to consider } \\
\text { explanatory variables. }\end{array}$ & Not reported & $\begin{array}{l}\text { Model used to consider } \\
\text { explanatory variables. }\end{array}$ \\
\hline & 1 & $\mathbf{0}$ & 2 \\
\hline \multirow[t]{2}{*}{ Were the analytical methods appropriate? } & $\begin{array}{c}\text { Inferential statistics } \\
\text { appropriate. }\end{array}$ & $\begin{array}{c}\text { Inferential statistics } \\
\text { appropriate. }\end{array}$ & $\begin{array}{c}\text { Inferential statistics } \\
\text { appropriate. }\end{array}$ \\
\hline & 2 & $\mathbf{2}$ & $\mathbf{2}$ \\
\hline \multirow[t]{2}{*}{ Was the precision of association given or calculable? Is association meaningful? } & $\mathrm{p}$ values reported. & $\mathrm{p}$ values reported. & $\mathrm{p}$ values reported. \\
\hline & 1 & 1 & 1 \\
\hline \multicolumn{4}{|l|}{ Section 5: Summary } \\
\hline \multirow[t]{2}{*}{ Are the study results internally valid (i.e. unbiased)? } & $\begin{array}{l}\text { Good reliability. Potential } \\
\text { for bias. Confounding } \\
\text { variables not addressed. }\end{array}$ & $\begin{array}{l}\text { Good reliability. Potential } \\
\text { for bias. Confounding } \\
\text { variables not addressed. }\end{array}$ & $\begin{array}{l}\text { Good reliability. Potential } \\
\text { for bias. Confounding } \\
\text { variables not addressed. }\end{array}$ \\
\hline & 1 & 1 & 1 \\
\hline \multirow[t]{2}{*}{ Are the findings generalisable to the source population (i.e. externally valid)? } & $\begin{array}{l}\text { Consecutive sampling, } \\
\text { source and population } \\
\text { reported }\end{array}$ & $\begin{array}{l}\text { Consecutive sampling, } \\
\text { source, eligibility and } \\
\text { population reported. }\end{array}$ & $\begin{array}{l}\text { Consecutive sampling, } \\
\text { source, eligibility and } \\
\text { population reported. }\end{array}$ \\
\hline & 1 & 2 & 2 \\
\hline Total Quality Score: & 20 & 21 & 23 \\
\hline External Validity (EV) score & 5 & 6 & 6 \\
\hline Internal Validity (IV) score & 15 & 15 & 17 \\
\hline
\end{tabular}


Table 1.6 Evaluation of a qualitative study using the SURE Quality Framework (Cardiff University,2012) for the expanded review.

\begin{tabular}{|c|c|}
\hline & Scoring: Yes, Can't tell, or No. \\
\hline & Panagopoulou et al. (2009) \\
\hline $\begin{array}{l}\text { Does the study address a clearly focused question/hypothesis? } \\
\text { Setting? } \\
\text { Perspective? } \\
\text { Intervention or Phenomena Comparator/control (if any)? } \\
\text { Evaluation/Exploration? }\end{array}$ & $\begin{array}{l}\text { Yes } \\
\text { Yes } \\
\text { Yes } \\
\text { N/A } \\
\text { Yes }\end{array}$ \\
\hline $\begin{array}{l}\text { Is the choice of qualitative method appropriate? } \\
\text { Is it an exploration of eg behaviour/reasoning/ beliefs)? } \\
\text { Do the authors discuss how they decided which method to use? }\end{array}$ & $\begin{array}{l}\text { Yes } \\
\text { Yes } \\
\text { No }\end{array}$ \\
\hline $\begin{array}{l}\text { Is the sampling strategy clearly described and justified? Is the sampling strategy clearly described and justified? } \\
\text { Is it clear how participants were selected? } \\
\text { Do the authors explain why they selected these particular participants? } \\
\text { Is detailed information provided about participant characteristics and about those who chose not to participate? }\end{array}$ & $\begin{array}{l}\text { Yes } \\
\text { Can't tell } \\
\text { No } \\
\text { Can't tell }\end{array}$ \\
\hline $\begin{array}{l}\text { Is the method of data collection well described? } \\
\text { Was the setting appropriate for data collection? } \\
\text { Is it clear what methods were used to collect data? } \\
\text { Type of method (eg, focus groups, interviews, open questionnaire etc) and tools (eg notes, audio, audio visual recording). } \\
\text { Is there sufficient detail of the methods used (eg how any topics/questions were generated and whether they were piloted; if } \\
\text { observation was used, whether the context described and were observations made in a variety of circumstances? } \\
\text { Were the methods modified during the study? If YES, is this explained? } \\
\text { Is there triangulation of data (ie more than one source of data collection)? } \\
\text { Do the authors report achieving data saturation? }\end{array}$ & $\begin{array}{l}\text { Yes } \\
\text { Can't tell } \\
\text { Yes } \\
\text { (Semistructured interview; audio) } \\
\text { Can't tell } \\
\text { No } \\
\text { No }\end{array}$ \\
\hline $\begin{array}{l}\text { Is the relationship between the researcher(s) and participants explored? } \\
\text { Did the researcher report critically examining/reflecting on their role and any relationship with participants particularly in relation to } \\
\text { formulating research questions and collecting data). } \\
\text { Were any potential power relationships involved (ie relationships that could influence in the way in which participants respond)? }\end{array}$ & $\begin{array}{l}\text { No } \\
\text { No } \\
\text { No }\end{array}$ \\
\hline
\end{tabular}


Is the data analysis/interpretation process described and justified?

Is it clear how the themes and concepts were identified in the data?

Was the analysis was performed by more than one researcher?

are negative/discrepant results taken into account?

\section{Are the findings credible?}

Are there sufficient data to support the findings?

Are sequences from the original data presented (eg quotations) and were these fairly selected?

Are the data rich (ie are the participants' voices foregrounded)?

Are the explanations for the results plausible and coherent?

Are the results of the study compared with those from other studies?

No

Can't tell

Can't tel

Can't tell

No

Yes

Can't tell

Can't tell

Yes

Yes

Yes

Yes

Yes

Yes

No

Did the authors identify any limitations?

Are the conclusions the same in the abstract and the full text? 


\subsubsection{Samples and populations}

All of the studies reviewed evidenced the prevalence of PTG in patients with myocardial infarction. Using the PTGI, Bluvstein et al. (2012) found that $71.2 \%$ of participants $(\mathrm{N}=82)$ reported PTG $(M=41.3 ; S D=27.3)$ : the most frequently reported change was appreciation of life and the least reported was spiritual change. Of note, Senol-Durak \& Ayvasik (2010) found that females ( $M=70.47 ; S D=20.2 ; N=19$ ) had significantly higher scores on the PTGI than males $(\mathrm{M}=56.5 ; \mathrm{SD}=26.7 ; \mathrm{N}=129)$ using the PTGI $(\mathrm{T}=2.22, \mathrm{df}=146, \mathrm{p}<.05)$. Garnefski et al. (2008) used the Personal Growth Scale (PGS) to measure PTG and also found evidence of PTG in patients with myocardial infarction $(M=15.46 ; S D=5.65)$ but did not report the percentage of participants who experienced growth. Using a qualitative methodology, Panagopoulou et al. (2009) reported that patients' experiences after myocardial infarction ( $N=11)$ were consistent with PTG. The following themes were identified: a catalyst for change/wake-up call, change of life philosophy, being protected/being invisible, and humanistic attitudes.

The inclusion and exclusion criteria used in several of the studies were alluded to but not made explicit (Garnefski et al. 2008; Panagopoulou et al. 2009). Senol-Durak and Ayvasik (2010) reported that potential participants were screened by cardiologists and nurses: patients who had a family history of other life-threatening illness were excluded from the study. Bluvstein et al. (2012) only included participants who had their first MI within the past 6 months, spoke Hebrew and had no history of mental health difficulties or other life-limiting illnesses.

All of the studies reviewed focused on adult populations with myocardial infarction with the mean age of participants ranging from 56.0 to 63.7. However, Panagopoulou et al. (2009) did not report the mean age of participants and instead reported that the age of participants ranged between 50 and 70 years. Mixed gender samples were used in all of the studies with the mean percentage of female participants ranging from $12.8 \%$ to $26.8 \%$ and the mean percentage of male participants ranging from $73.2 \%$ to $87.2 \%$. Again, Panagopoulou et al. (2009) did not explicitly state how many participants were male and female. Garnefski et al. (2008) reported a response rate of $65 \%$ ( $N=22$ refused to participate, $n=14$ rejected participating after aims were explained) whereas Bluvstein et al. (2012) reported a response rate of $73.2 \%$ ( $\mathrm{N}=30$ refused to participate). 
The studies reviewed recruited from a diverse range of populations in different countries (i.e. Bluvstein et al. (2012): 100\% of participants were identified as of 'white ethnicity', Israel; Garnefski et al. 2008: ethnicity not specified, The Netherlands; Panagopoulou et al. 2009: ethnicity not specified, Greece; Senol-Durak \& Ayvasik (2010): ethnicity not specified, Turkey) which reflect the clinical reality of stroke being a world health issue (WHO, 2012) but limits the generalisability of the findings. It is unclear whether other socio-demographic data were recorded and of note, none of the studies reviewed used a UK population.

In terms of the role of time since event, Garnefski et al. (2008) report that 'post-MI time' was used in analysis but such data are not reported in the paper. Bluvstein et al. (2012) recruited participants a maximum of 6 months since the event. Senol-Durak \& Ayvasik (2010) reported the 'time passed since diagnosis' ( $M=1371.7$ days; SD=239.5) Panagopoulou et al. (2009) recruited participants who had been an inpatient during a fixed period of time.

\subsubsection{Design and recruitment}

All of the studies reviewed employed a cross-sectional design. Two studies used structural equation analysis to examine the role of specific variables on PTG (Garnefski et al. 2008; Senol-Durak \& Ayvasik, 2010)) but used different measures of PTG. Bluvstein et al. (2012) employed a cross-sectional, survey design to investigate the relationship between PTG and posttraumatic stress symptoms (PTSS) and in particular, whether PTG moderates the association between PTSS and mental health. The reliance on cross-sectional design in these studies limits the possibility of making any inferences regarding the causal relationship between PTG and myocardial infarction. One study employed a qualitative methodology using interpretative phenomenological analysis to examine the process of dyadic benefit finding after myocardial infarction. Although participants were recruited consecutively within a specific timeframe and the research question was clear, this study did not report a welldefined study protocol nor did it explicitly state the use of inclusion and exclusion criteria.

All studies recruited participants through clinical settings (e.g. cardiology outpatient clinic, cardiac rehabilitation unit), although exclusion criteria and response rate were not made clear in all of the studies. Two studies used consecutive sampling (Bluvstein et al. 2012; Panagopoulou et al. 2009), whereas the other two studies used convenience sampling (Senol- 
Durak \& Ayvasik, 2010; Garnefski et al. 2008). However, the studies which employed convenience sampling failed to comment on the generalisability of the finding to the broader population. None of the studies were longitudinal or control matched studies. There was also inconsistency across the measures of PTG used.

\subsubsection{Sample size}

The sample sizes used in the studies reviewed varied considerably (see Table 1.4). Of note, none of the studies reviewed documented statistical power.

\subsubsection{Treatment of confounding variables}

The studies reviewed controlled for a range of confounding variables in the design and included factors such as multiple myocardial infarctions (Garnefski et al. 2008), history of other life-limiting illness in participant or family members (Senol-Durak \& Ayvasik, 2010; Bluvstein et al. 2009) and mental health difficulties (Bluvstein et al. 2009). All of the studies reviewed alluded to controlling for the potential confounds of co-morbid disease and mental health difficulties, but few studies clearly reported inclusion and exclusion criteria. None of the studies reported that the effects of potential confounding factors were tested and adjusted.

\subsubsection{Review of study findings}

All of the studies reviewed demonstrated evidence of PTG in myocardial infarction (MI) survivors (Bluvstein et al. 2012; Garnefski et al. 2008; Panagopoulou et al. 2009; Senol-Durak \& Ayvasik, 2010). The findings of the studies reviewed support the wider literature which has found evidence of PTG in trauma survivors of a similar age range (e.g. Cordova et al. 2011; Kuenemund et al. 2014).

In terms of the question underpinning this systematic review, these studies provide some good quality findings, albeit limited in nature, regarding the predictors of PTG in MI survivors. One study found that posttraumatic stress symptoms (PTSS) were positively associated with PTG and additionally, they found PTG moderated the relationship between PTSS and mental health outcomes (Bluvstein et al. 2012). These findings point toward the role of PTSS in PTG and support the findings reported by Kuenemund et al. (2014) that distress and growth can 
co-exist in stroke survivors (see section 1.5.2.7). However, the temporal nature of PTSS and PTG post-stroke is unclear. As such, the findings reported by Bluvstein et al. (2012) are somewhat limited by the reported range of time since MI (i.e. maximum of six months post$\mathrm{MI}$ ), as well as the use of a correlational design which could not establish the direction of causality between PTG and PTSS.

Senol-Durak and Ayvasik (2010) found that coping was positively related to PTG, but perception of the traumatic event was not associated with PTG. Of note, they also found that perceived social support was positively related to coping and perception of the traumatic event. Furthermore, using structural equation analysis, they found that perceived social support was significantly related to PTG through the effect of coping. Such findings provide support for the model of PTG proposed by Schaefer and Moos (1992). A key strength of the study reported by Senol-Durak and Ayvasik (2010) is that they attempted to test the mechanisms which explain the associations between the variables under investigation and in doing so, contributed to the empirical support for Schaefer and Moos' model of PTG (1992).

Garnefski et al. (2008) found that psychological health and personality were predictive of PTG, but reported that cognitive coping strategies (i.e. putting into perspective, positive reframing and positive reappraisal) were most predictive of PTG. These findings are consistent with those reported in the stroke survivor literature (Gangstad et al. 2009) and provide support for the conceptualisation of PTG proposed by Tedeschi and Calhoun $(1995,2004)$. The study reported by Garnefski et al. (2008) had a much larger sample size and a more limited range of time since traumatic event (i.e. 3-12 months compared to 5-99 months) compared to Gangstad et al. (2009). One study found that participants who experienced PTG conceptualised it as a deliberate coping process (Panagopoulou et al. 2009). While such findings provide cursory support for the mechanism underpinning PTG, Panagopoulou et al. (2009) do not account for how effortful, deliberate cognitive processing facilitates PTG (Tedeschi \& Calhoun, 2004).

In summary, there is evidence to suggest that PTSS are associated with the development of PTG in MI survivors which is somewhat consistent with studies of stroke survivors (Bluvstein et al. 2002; Kuenemund et al. 2014). Such findings point toward the co-existence of PTG and PTSS but do not provide evidence regarding the direction of causality. One study found 
evidence in support of the model of PTG proposed by Schaefer and Moos (1992). Senol-Durak and Ayvasik (2010) found that perceived social support was significantly related to PTG through the effect of coping. They also found that perceived social support was associated with coping and perception of the traumatic event.

Garnefski et al. (2008) found that PTG was predicted by psychological health and personality, but reported that cognitive coping strategies were most predictive of PTG. The role of cognitive processing in PTG has also been demonstrated in the stroke survivor literature (Gangstad et al. 2009) but the study reported by Garnefski et al. (2008) appeared to be of a higher quality due to sample size and a more conservative range of time since MI. While there are some qualitative findings which point toward the role of deliberate coping in PTG, further research is required to determine the precise nature of deliberate coping, as well as the temporal relationship between coping and PTG.

Despite the aforementioned strengths and shortcomings of the studies already discussed, a range of other factors impacted on the overall quality of the findings presented. Most notably, the studies reviewed used different measures of PTG and were conducted in different countries which impacts on the generalisability of the findings. Although the studies reported that attempts were made to reduce confounding factors at the design phase, efforts to test for potential confounding effects were not reported. Furthermore, the reliance on using a cross-sectional methodology in these studies limits the causal inferences which could be made between PTG and MI, as well as our understanding of the developmental trajectory of PTG.

\subsubsection{Overall Summary}

This section summarises the key findings and factors pertaining to quality for the studies reviewed in the initial systematic review and the expanded systematic review. All of the studies reviewed demonstrated evidence of PTG albeit using different measures and research methodologies. Emerging findings in the literature show comparable levels of PTG in stroke survivors relative to other trauma survivors (Kuenemund et al. 2014). All of the studies reviewed were cross-sectional which has implications for how the variables identified as 
predictors of PTG can be interpreted, as the direction of causality cannot be identified. This also has implications for how the findings can be interpreted in light of the proposed theoretical models of PTG (Schaefer \& Moos, 1992; Tedeschi \& Calhoun, 1995, 2004) given the lack of findings regarding the temporal relationship between PTG and predictor variables over time. In addition, only one study reviewed attempted to investigate the mechanism of PTG proposed by Tedeschi and Calhoun $(1995,2004)$ (Senol-Durak \& Ayvasik, 2010) and none of the studies reviewed examined the mechanisms proposed to mediate PTG in stroke survivors.

Most studies attempted to control for the role of confounding factors in the design phase, but few reported how the role of potential confounding factors were identified and adjusted for apart from Kuenemund et al. (2014). Of note, a strength of the studies reviewed is that most studies recruited participants from clinical settings. However, it is likely that the diversity of sampling across the studies reviewed limits the potential generalisability of the findings to a UK setting. Nevertheless, the studies reviewed identified that PTG was positively associated with cognitive processing (Gangstad et al. 2009), event centrality (Kuenemund et al. 2014), and negatively associated with depression (Gangstad et al. 2009) in stroke survivors. More specifically, Gangstad et al. (2009) found that as time since stroke increased, the relationship between PTG and two aspects of cognitive processing (i.e. downward comparisons and resolution) became more positive and significant. This finding suggests that a change in cognitive processing and the outcomes of such processing (i.e. perceived growth) may be involved in the development of PTG over time In a study of MI survivors, Senol-Durak \& Ayvasik (2010) found that perceived social support was significantly related to PTG through the effect of coping. Further research is needed to examine the mechanisms which may underpin PTG in stroke survivors. The studies reviewed on PTG in MI survivors lend partial support to the predictors of PTG found in stroke survivors and identify other predictors of PTG such as PTSS (Bluvstein et al. 2012), coping (Senol-Durak \& Ayvasik, 2010), psychological health (Garnefski et al. 2008), personality (Garnefski et al. 2008) and cognitive coping strategies (Garnefski et al. 2008). 


\subsection{Rationale for the thesis and hypotheses}

This chapter has explored the construct of posttraumatic growth (PTG) within the context of different theoretical frameworks and traumatic events. The dominant models of PTG in the literature posit different mechanisms through which growth occurs (Schaefer \& Moos, 1992; Tedeschi \& Calhoun, 1995, 2004). Schaefer and Moos (1992) propose that multiple factors (i.e. event-related, environmental, personal, cognitive and coping) interact in predicting PTG and stipulate that approach coping, a combination of active coping and cognitive processing, predict PTG as opposed to avoidance or denial coping. In contrast, Tedeschi and Calhoun (1995, 2004) have developed a cognitively-oriented model of PTG which proposes that rumination, social support and acceptance coping are predictive of PTG. They propose that it is the individual's struggle with the new reality in the aftermath of trauma and their cognitive processing, or deliberate rumination, therein that determines the extent to which PTG occurs. According to the literature, PTG can occur from within a few weeks to a few years following exposure to a traumatic event (Tedeschi \& Calhoun, 2004).

As discussed, there is a dearth of literature examining PTG in stroke survivors, where the few studies conducted have been cross-sectional in design (e.g. Gangstad et al. 2009) which limits the conclusions that can be drawn. The absence of longitudinal studies examining PTG in the stroke literature reflects the limited empirical evidence base for the predictors of PTG as proposed by models of PTG (Schaefer \& Moos, 1992; Tedeschi \& Calhoun, 2004). Nevertheless, there is emerging evidence regarding the role of rumination in predicting PTG in stroke survivors (Gangstad et al. 2009), the role of mood related factors in facilitating PTG post-stroke (Kuenemund et al. 2014), as well as the ability to integrate the traumatic experience post-stroke with self-identity (Kuenemund et al. 2014). Comparable literature in myocardial infarction (MI) survivors supports the findings in the stroke literature and provides evidence for the predictive utility of Schaefer and Moos' model of PTG (1992) (Senol-Durak \& Ayvasik, 2010). Other studies of PTG in MI survivors have indicated the role of posttraumatic stress symptoms (Bluvstein et al. 2012), coping (Senol-Durak \& Ayvasik, 2010), psychological health (Garnefski et al. 2008), personality (Garnefski et al. 2008), deliberate coping (Panagopoulou et al. 2009) and rumination (Garnefski et al. 2008). In a study of stroke carers, Hallam and Morris (2014) found that deliberate rumination was the greatest predictor of PTG 
but also, that deliberate rumination mediated the effect of social support on PTG which supports the model of PTG proposed by Tedeschi and Calhoun (2004).

Using a longitudinal survey design with two points in time separated by six months, this study aims to address the current gap in the literature regarding the predictors of PTG in stroke survivors over time. Consistent with the models of PTG outlined in this chapter (Schaefer \& Moos, 1992; Tedeschi \& Calhoun, 1995, 2004), the following hypotheses were made:

1a) Stroke survivors will report significantly more PTG at time 2 compared to time 1.

1b) The PTG stroke survivors report will not be significantly different from the PTG reported by other trauma survivors in the literature.

2a) PTG at time 2 will be positively associated with social support, active coping and daily functioning at times 1 and 2 .

2b) PTG at time 2 will be positively associated with acceptance coping at time 2 .

2c) PTG at times 1 and 2 will be negatively associated with denial coping at times 1 and 2.

2d) Social support at times 1 and 2 will be positively associated with rumination, active coping, daily functioning, acceptance coping at times 1 and 2, but negatively associated with denial coping at times 1 and 2 .

2e) Active coping and acceptance coping at time 1 will mediate the relationship between social support at time 1 and PTG at time 2 .

3a) Rumination at time 1 will be positively associated with posttraumatic stress symptoms (PTSS) at time 1 and negatively associated with denial coping at time 1. 
3b) Rumination at time 2 will be positively associated with PTSS and Acceptance coping at time 2, but negatively associated with denial coping at time 2 .

3c) Acceptance coping at time 2 will be negatively associated with PTSS at time 2 .

3d) PTGI at time 1 will be associated with rumination and PTSS at time 1.

3e) PTGI at time 2 will be association with rumination at times 1 and 2, as well as PTSS at time 2.

3f) Rumination at time 1 will mediate the relationship between PTSS at time 1 and PTG at time 2

3g) Rumination at time 1 will mediate the relationship between PTSS at time 1 and PTG at time 1.

3g) Rumination at time 1 will mediate the relationship between social support at time 1 and PTG at time 2. 


\title{
Chapter Two
}

\author{
Methodology
}

\subsection{Design}

This study employed a longitudinal survey design to explore the relationships between the dependent variable (DV) posttraumatic growth (PTG) and the independent variables (IV): social support, rumination, posttraumatic stress, coping and activities of daily living. All participants were asked to complete standardised measures of these variables: the Post Traumatic Growth Inventory (PTGI; Tedeschi \& Calhoun, 1996), the Active Coping, Denial and Acceptance subscales of the COPE scale (Carver et al. 1989), the Multidimensional Scale of Social Support (MSPSS; Zimet et al. 1988), the Rumination Scale (Calhoun et al. 2000), the Barthel Index (Mahoney \& Barthel, 1965) and the Impact of Events Scale Revised (IES-R; Weiss \& Marmar, 1997). Correlations, multivariate regression and mediation analysis were used to explore the relationships between PTG, social support, rumination, posttraumatic stress, coping and activities of daily living.

\subsection{Rationale for methodology}

A quantitative methodology was chosen in order to address the research questions identified and to determine the nature of the relationships between the variables of interest. Using a quantitative methodology allowed these variables to be measured in a standardised way and for the findings to be considered in light of relevant theoretical models (e.g. Tedeschi \& Calhoun, 2004; Schaefer \& Moos, 1998) and the existing literature. A longitudinal survey design, rather than a cross-sectional design, was employed in order to identify the variables associated with the development of PTG over time. The use of closed-ended questions (e.g. dichotomous, multiple choice and scaled questions) produced data points which facilitated the use of statistical methods of analysis. 
Although a qualitative methodology would have provided data rich with 'personal meaning' regarding PTG in stroke survivors (Hanson et al. 2003), there is literature which has already explored the phenomenon of PTG in stroke survivors (Gillen, 2005) and related conditions (e.g. myocardial infarction; Panagopoulou et al. 2009). Therefore, a quantitative methodology was chosen as it provided a means of quantifying the variables of interest and provided a way of examining the process by which such variables mediate PTG in stroke survivors. Furthermore, employing a quantitative methodology facilitates the replicability of the study and potentially allows the study findings to be generalised from a sample to a wider population (Babbie, 1990). Unlike a qualitative methodology, quantitative methods provide a way to establish the process by which different variables are related and methods such as mediation analysis enable multiple simultaneous mediator variables to be investigated (Preacher \& Hayes, 2008).

\subsection{Participants}

\subsubsection{Power analysis}

An assessment of statistical power was conducted using G*Power software (Faul et al. 2007) to determine an appropriate sample size for this study. The PTG literature has demonstrated correlations between PTG and independent variables (rumination, active coping, acceptance coping, social support, age) range from .29 to .46. Based on Cohen (1988, pp.101-102), at least 69 participants were needed to detect similar correlations with alpha at .05 and power at .80. For multiple regressions with five predictors, a sample size of 70 would permit detection of an effect size ( $\mathrm{f} 2$ ) as small as .091 at a power of .80 with alpha set at .05. An attrition rate of $25 \%$ over 12 months was predicted, therefore the required number of participants to was increased to 93.

\subsubsection{Inclusion and Exclusion criteria}

All participants were over 18 years of age and had the capacity to provide informed consent. Potential participants were eligible for inclusion in the study if they had experienced one known stroke and were between 2 and 6 months post stroke at the time of recruitment. 
Potential participants were excluded if they were experiencing cognitive difficulties to a degree which would have impacted on their ability to provide informed consent and complete the questionnaires. Potential participants were also excluded if they had previously experienced a stroke and also, if they were pregnant. Only eligible participants who provided consent participated in the study. One participant was excluded from the study at follow-up due to her stroke diagnosis having been disconfirmed. The frequency of potential participants who were eligible to participate and declined to participate was not recorded. This was due to the first point of contact with potential participants being made by the clinical team, the researcher only accessed details regarding the participants who were interested in participating.

\subsection{Procedure}

\subsubsection{Participant recruitment}

The difficulties associated with recruiting clinical groups who have chronic conditions has been widely documented in the literature (e.g. Townsley et al. 2005). In terms of stroke survivors, Hadidi et al. (2012) have found that the challenges associated with the recruitment and retention of this clinical group centres on the complex health needs and post stroke sequelae often experienced by stroke survivors. They recommend being accommodative to the schedules of stroke survivors and suggest establishing realistic inclusion and exclusion criteria. This literature was considered when designing the recruitment strategy for this study.

Stroke survivors were recruited through Cardiff and Vale University Health Board with the support of the Consultant Physician for Acute Stroke at the University Hospital of Wales, the department of Clinical Gerontology and the Research and Development department. With the support of a medical secretary, the Consultant Physician identified potential participants who met the inclusion criteria prior to their two month post stroke follow-up appointment and an information sheet about the study was posted to the potential participants (see Appendix C). This information sheet was accompanied by a cover letter which explained that there would be a researcher available at the clinic following their appointment should they be interested in participating and have any questions about the research (see Appendix D). 
Prior to clinic starting, the Consultant Physician and associated medical doctors were reminded of the inclusion and exclusion criteria for the study and were asked to invite the stroke survivors who had been sent information sheets to participate in the study. They were also asked to ensure that potential participants understood that their decision to participate, or not, would in no way impact on their care or treatment.

However, due to a low level of participant recruitment, the recruitment procedure was amended. The Consultant Physician screened suitable potential participants and a medical secretary was funded to contact potential participants by telephone informing them of the study and asking if they would like the researcher to contact them with further information about the study. Potential participants who consented to being contacted by the researcher were posted information sheets (see Appendix E) and telephoned a few days later to enquire whether they were interested in participating. The researcher was accommodative to the schedules of participants and visited them at home at a mutually convenient time.

\subsubsection{Consent}

The researcher engaged potential participants in a process of informed consent prior to participating. Potential participants were provided with information sheets (see Appendix C and E), given at least 72 hours to consider whether they wanted to participate and invited to ask questions about the research. The Consultant Physician screened potential participants for capacity to consent and cognitive difficulties which may interfere with ability to participate. However, the researcher also ensured that participants had the capacity to consent at the time of participation. Participants were asked to sign a consent form to show that they understood the information sheet and agreed to take part in the research (see Appendix $F$ and $G$ ). All participants were informed both in writing (see Appendix $C$ and $E$ ) and verbally that they were free to withdraw from the study at any time without any consequences. 


\subsubsection{Data collection and storage}

Data collection took place between May 2014 and March 2015 and occurred in an outpatient setting or in participants' homes. Follow-up data were collected over the telephone. Participants were given the opportunity for a copy of the questionnaire to be posted to them to aid data collection over the telephone. All participant data were stored using randomly allocated anonymised participant identifiers and information linking these identifiers to participant names was stored in a locked cabinet accessible only to the researcher and academic supervisor. Identifying information was destroyed once the participant completed their involvement in the study. All data collected will be stored for 15 years using a locked cabinet in line with Cardiff University guidelines (http://www.cardiff.ac.uk/govrn/cocom/accinf/dataprotection/index.html). Participant data were kept confidential unless a participant disclosed information which caused the researcher to be seriously concerned for the participant's safety, or for the safety of someone else (see section 2.4.4.2. for further information).

\subsubsection{Ethical considerations}

\subsubsection{Ethical approval}

The requirements for ethical approval of this study were considered in terms of the National Research Ethics Committee (NRES) criteria for ethical review (http://www.hra.nhs.uk), as well as the ethical implications of participant recruitment within the NHS. It was determined that the study needed ethical approval from a NRES Research Ethics Committee (REC) (http://www.hra-decisiontools.org.uk/ethics/). Ethical approval of the study was granted by a local NRES REC (see Appendix H).

As the research was conducted in the NHS in Wales, it also required approval by other bodies such as the Research and Development department at Cardiff and Vale University Health Board (UHB) as well as the National Institute for Social Care and Research (NISCHR) (see Appendix I for approval process flowchart). The study was sponsored by Cardiff University (see Appendix J) as per local agreement protocols for students on the Doctoral Programme in Clinical Psychology. However, as the research was conducted in Cardiff and Vale UHB, a 
further review process was conducted by the Research and Development department prior to the study being granted approval (see Appendix K). The researcher ensured approval was granted from all relevant bodies prior to commencing the study.

Due to the difficulties with participant recruitment, the study design was amended in an effort to optimise potential participant recruitment. A formal amendment was submitted to the REC for consideration (see Appendix L for summary of changes) and approval for the amended study was granted (see Appendix M).

\subsubsection{Participant wellbeing}

The process of gaining informed consent involved explaining the limits of confidentiality to potential participants. It was explained that if a participant disclosed information which caused the researcher concern regarding the participant's own safety or those around them, then the researcher would need to inform other professionals of their concerns. Accidental disclosures were also managed within the limits of confidentiality and participants were reminded of the researcher's role relative to the potential role of other health professionals in their care.

The content of the questionnaires used in this study had the potential to lead to participant distress or discomfort. To minimise the effect of potential participant distress, the possibility of experiencing distress was clearly stated in the information sheet (see Appendix C and Appendix E) and participants were reminded that they could withdraw from the study at any point without any consequences. Participants who experienced distress while participating were offered reassurance and the option to discontinue. Participants who experienced distress while participating and requested input regarding their mental wellbeing were advised to discuss their concerns with their General Practitioner and signposted toward charities such as MIND and the Stroke Association where peer support and counselling are available on a self-referral basis.

Participants were provided with the researcher's contact details should they have any further questions or concerns regarding their participation in the study. Participants were also given the opportunity to get feedback on the outcome of the study in written form. 


\subsubsection{Researcher wellbeing}

Most participants were seen in their own homes. The study was carried out in accordance with the Cardiff and Vale UHB lone worker policy (see Appendix N). The researcher utilised academic supervision to consider any other issues pertaining to researcher wellbeing.

\subsection{Measures}

The Stroke Survivors' Survey consisted of a demographic survey, as well as a battery of validated questionnaires (see Appendix O).

\subsubsection{Demographic Survey}

Participants were asked to complete a demographic survey which contained items relating to individual factors such as participant age, sex, ethnicity, occupation, mood, cognitive functioning, as well as items related to stroke specific factors. Items were identified following a literature search and consultation with the researcher's academic supervisor who is experienced in conducting research using stroke survivors. Participants were asked, for example, if they were feeling anxious or depressed as these mood related variables can be associated with the development of PTG (see Zoellner \& Maercker, 2006).

\subsubsection{Posttraumatic growth}

The Posttraumatic Growth Inventory (PTGI; Tedeschi \& Calhoun, 1996) is a 21 item self-report inventory which uses a six-point Likert scale to measure positive outcomes that result from traumatic experiences. The Likert scale ranged from 0 ('I did not experience change') to 5 (' experienced this change to a very great degree'). The PTGI produces an overall scale score which ranges from 0-105, whereby any score over 0 represents growth. However, the PTGI also comprises of 5 different factors related to growth: Relating to Others, New Possibilities, Personal Strength, Spiritual Change, and Appreciation of Life.

The PTGI has 'very good' internal consistency (total scale alpha $=0.90-0.94$ ) and acceptable internal consistency for the subscales (Cronbach's alpha $=0.67-0.85)$ (Tedeschi \& Calhoun, 
1996). The test-retest reliability of the PTGI has been demonstrated to be within the 'acceptable' range using a sample of undergraduate students $(r=.71$, Tedeschi \& Calhoun, 1996). Although Tedeschi and Calhoun (1996) have demonstrated that the PTGI is unrelated to a measure of social desirability (i.e. the Marlowe, Crowne Social Desirability Scale; Crowne \& Marlowe, 1960), the validity of the scale has been criticised for its lack of key operational definitions (Bitsch et al. 2011). However, the PTGI has previously been shown to have 'excellent' internal consistency (Cronbach's alpha $=0.92)$ when used with a sample of stroke survivors (Gangstad et al. 2009). The PTGI was therefore deemed to be a sufficiently reliable and valid measure for this study.

\subsubsection{Coping}

The COPE scale (Carver et al. 1989) is a 60 item self-report inventory of coping resources considered both functional and dysfunctional with 15 different subscales (Carver et al. 1989 for psychometric properties). Consistent with the PTG literature (e.g. Bellizzi et al. 2006), only subscales of interest were used in this study: the active coping scale (i.e. problem-focused coping), as well as the denial and acceptance scales (i.e. emotion-focused coping). Carver et al. (1989) demonstrated 'acceptable' internal consistency for the active coping and acceptance scales (Cronbach's alpha $=0.62$ and 0.65 respectively) but the internal consistency of the denial scale was with the 'good' range (Cronbach's alpha $=0.71)$. This is consistent with the use of scales from the COPE in the PTG literature (e.g. Park et al. 2005; Cronbach's alpha $>0.65$ ). However, test-retest reliability was 'poor' for active coping and denial ( $r=0.56$ and 0.54 respectively) and 'questionable' for acceptance $(r=0.63)$ using a student sample (Carver et al. 1989). Given that the COPE scale is applicable to both situational coping and dispositional coping style, it is probable that coping strategies vary over the course of a stressful period such as post-stroke adjustment. This is somewhat at odds with the assumption underlying test-retest reliability that the structure of the concept measured does not change over time (Menard, 2007). Therefore, three scales of the COPE were chosen for this study on the basis of their internal consistency, ecological validity and compatibility with the theoretical models examined. 
The COPE scale does not produce an overall score. Specific subscales can be used and analysed relative to other variables (http://www.psy.miami.edu/faculty/ccarver/ sclCOPEF.html). Analysis can also involve creating second-order factors from the scales and using the factors as predictors (Carver et al. 1989).

\subsubsection{Social Support}

The Multidimensional Scale of Social Support (MSPSS; Zimet et al. 1988) is a 12 item selfreport inventory which uses a seven-point Likert scale to measure perceived social support. The Likert scale ranged from 1 ('Very strongly disagree') to 7 ('very strongly agree). The MSPSS produces a total score which ranges from 12 to 84 , where a higher score indicates higher perceived social support. However the MSPSS also produces subscale scores for perceived support from family, friends and significant others. The MSPSS has demonstrated 'excellent' internal consistency (Cronbach's alpha $=0.87-0.94)$ and 'acceptable' test-retest reliability $(r$ $=.73$ ) using a sample of older adults (Stanley et al. 1998).

\subsubsection{Rumination}

The Rumination Scale (Calhoun et al. 2000) is a 14 item self-report inventory which measures rumination style following a stressful experience. Participants respond using a four-point scale ranging from 'not at all' to 'often'. The items in this inventory reflect both intrusive deliberation (e.g., 'Thoughts about the experience came into my mind and I could not get rid of them') and deliberate rumination (e.g., 'I have tried to make something good come from my struggle'). Participants are asked to reflect on their level of rumination shortly after the event and more recently. The Rumination Scale produces an overall score, whereby a higher score indicates a higher level of rumination.

Calhoun et al. (2000) demonstrated 'good' internal consistency for the scale using a general US population for intrusive rumination (i.e. soon after the event: Cronbach's alpha $=0.85$; recently: Cronbach's alpha $=.83$ ) and deliberate rumination (i.e. soon after the event: Cronbach's alpha $=.72 ;$ recently: Cronbach's alpha $=.74)$ (Taku et al. 2009). Test-retest 
reliability for total scale scores have been found to be 'acceptable' ( $r=0.71$; Calhoun et al. 2000).

\subsubsection{Level of functioning}

The Barthel Index (Mahoney \& Barthel, 1965) is a 10-item self-report inventory which measures an individual's functioning on a range of different day-to-day activities or 'activities of daily living' which include dressing, personal care and mobility (Colin et al. 1988). It is a selfreport scale where participants report the extent to which they can perform specific activities of daily of living using a fixed range of responses. A systematic review and meta-analysis have demonstrated that the Barthel Index has excellent reliability as an outcome after stroke (Duffy et al. 2013).

\subsubsection{Posttraumatic stress}

The Impact of Events Scale Revised (IES-R; Weiss \& Marmar, 1997) is a 22-item self-report inventory which measures subjective distress following a traumatic event. The IES-R is a revised version of the IES which contains 12 items (Horowitz et al. 1979). The IES-R contains items which relate to intrusion, avoidance and hyperarousal. Participants are asked to think about a specific traumatic event and indicate whether they experienced distress over the past 7 days using a 5-point Likert scale which range from 0 ('Not at all') to 4 ('Extremely'). The IES$\mathrm{R}$ produces a total score which ranges from 0-88 and subscale scores can be computed for the intrusion, avoidance and hyperarousal. This scale has previously been demonstrated to have high levels of internal consistency in people who have experienced a traumatic event (Intrusion scale: Cronbach's alpha $=0.87-0.94$; Avoidance: Cronbach's alpha $=0.84-0.87$; Hyperarousal: Cronbach's alpha $=0.79-0.91$ (Creamer et al. 2005). The test-retest reliability of the IES-R has been demonstrated to be strong using a combined sample of emergency worker and earthquake survivors ( $r=0.89, r=0.94$; Weiss \& Marmar, 1997). 


\subsection{Statistical Analysis}

\subsubsection{Univariate and multivariate analyses}

The statistical software SPSS version 20 (IBM Corporation, 2011) was used to conduct data analysis. Prior to analysis, the distribution of all continuous data were inspected for outliers and deviation from a normal distribution, and checked for equality of variance to determine whether the assumptions for parametric testing were met (see section 3.2). As a result of this process, a bootstrapping module in SPSS was used in the following statistical analyses: bivariate correlation, t-test and multiple regression. Bootstrapping involves estimating the properties of the sampling distribution from the sample data and it produces percentile bootstrap confidence intervals based on the values between which $95 \%$ of bootstrap sample estimates fall (Field, 2013) (see section 3.2 for further detail).

Attempts were made to control for confounding effects at the design stage of this research (e.g. cognitive difficulties, previous stroke). However, bivariate correlations, t-tests and ANOVA were conducted to determine whether there any confounding relationships between the demographic variables and the main study variables. Independent samples t-tests were calculated by hand to establish whether mean PTGI scores for a post stroke population differed from mean PTGI scores documented in the literature. Paired samples t-tests were used to determine whether the difference between variables at time 1 and time 2 were significant. Bivariate correlation analyses was used to determine the relationship between variables of interest over time.

Multiple regression analyses were used to identify the predictors of PTG over time. Prior to completing regression analyses, scatter plots and residuals were inspected to ensure that the relationships between the PTGI scores and the dependent variables were linear and to check for homoscedasticity. As recommended by Field (2013), the assumption for absence of multicollinearity was assessed, as was the assumption for independent errors using the DurbinWatson statistic. Hierarchical linear regression was used to assess the relative utility of two models of PTG (i.e. Schaefer \& Moos, 1992; Tedeschi \& Calhoun, 1995, 2004) in predicting PTG in this sample. An exploratory stepwise linear regression was also conducted in order to determine which factors were most predictive of PTG. 


\subsubsection{Mediation analysis}

Traditional approaches to mediation analysis (Baron \& Kenny, 1986) have been criticised for lacking power (Mackinnon et al. 2002) and for not directly testing mediation (Hayes, 2009). Approaches such as the Sobel test (Sobel, 1982) have attempted to address these weaknesses but make parametric assumptions (Lockwood \& Mackinnon, 1998). Bootstrapping approaches provide a non-parametric way to conduct mediation analysis and have been demonstrated to have greater power and more accurate Type-1 error than the other approaches, particularly in small samples (Williams \& Mackinnon, 2008).

This study conducted mediation analysis with bootstrapping approaches using PROCESS (Hayes, 2013) to determine the ways in which the different variables measured were related to PTGI scores. The mediation analysis was carried out in SPSS using PROCESS (Hayes, 2013; http://www.afhayes.com/spsssas-and-mplus-macros-and-code.html). This non-parametric bootstrap approach to conducting mediation analysis involves directly testing the significance of the mediating or indirect effects. As Figure 2.1 shows, the total effect of $X$ on $Y$ is path ' $c$ '. When a mediator, $M$, is added, this allows the ' $a$ ' coefficient for $X$ to be calculated and the ' $b$ ' coefficient for $Y$ to be calculated (Figure 2.1). The $c^{\prime}$ coefficient represents the direct effect of $X$ on $Y$ when ' $a$ ' and ' $b$ ' are controlled for, whereas the product of ' $a$ ' and ' $b$ ' amounts to the indirect effect of $X$ on $Y$ through $M$ (Baron \& Kenny, 1986). The mediation or indirect effect represents the difference between the total and direct effect of $X$. Although this approach does not produce $p$-values, the null hypothesis can be rejected at the $p<.05$ level of significance if the lower and upper bounds of the $95 \%$ confidence intervals do not cross zero.

Of note, Hayes (2009) recommends that no simple association between two variables is necessary when testing hypotheses about the role of potential indirect or mediating effects. Simple mediation analysis were selected over a multiple-mediator model as including multiple predictors can lead to instability of regression coefficients and it also enabled specific hypotheses to be tested (Hayes, 2013). In this study, mediation analyses were used to test specific hypotheses based on the theoretical models of PTG (i.e. Schaefer \& Moos, 1992; Tedeschi \& Calhoun, 1995, 2004). More specifically, mediation analyses were used to test whether there was an indirect effect of rumination on the relationship between PTSS and 
PTG. It was also used to test whether there was an indirect effect of type of coping on the relationship between social support and PTG.

\section{Total effect of $X$ on $Y$ model:}

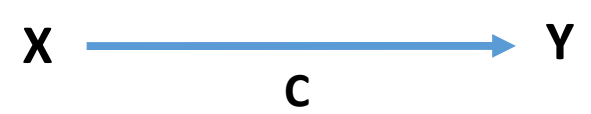

Simple mediation model of the direct $\left(c^{\prime}\right)$ and indirect effects $(a b)$ of $X$ on $Y:$

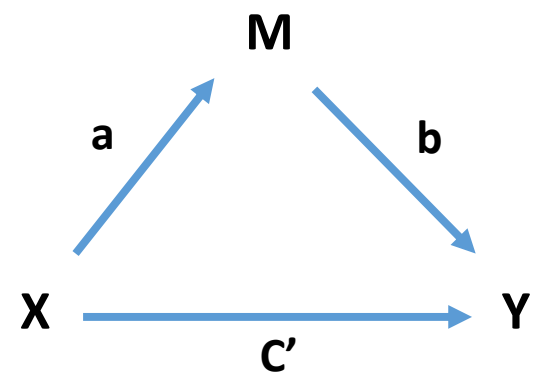

Figure 2.1 Total effect of $X$ on $Y$ model and a simple mediation model illustrated

\subsubsection{Multiple testing effects}

It was decided not to use Bonferroni corrections based on the literature which suggests that Bonferroni corrections are highly conservative and may miss significant relationships, particularly when evaluating the relationships between different variables in survey based research (Bland, 1996). Furthermore, the literature reviewed in the systematic reviews did not report the use of such statistical corrections. In the case of this study, the number of tests conducted was not seen as relevant to interpreting the differences between variables at time 1 and time 2. Furthermore, Perneger (1998) recommends describing what tests have been performed and why as the best way of dealing with multiple comparisons. Therefore, no Bonferroni corrections were performed. Instead, a clear rationale has been presented for what tests have been performed and why. 


\title{
Chapter Three
}

\author{
Results
}

\subsection{Overview}

In this chapter, an account of the data cleaning process and the testing of assumptions for parametric testing is given. This is followed by descriptive analyses of the main clinical and demographic study variables. The third section explores and outlines the characteristics of posttraumatic growth (PTG) in this sample. The fourth, fifth, sixth and seventh sections show the results of the inferential analyses used to test the main research questions and hypotheses.

\subsection{Data cleaning and assumptions for parametric testing}

The data were explored to determine whether the assumptions for parametric statistics were met. This process involved visually inspecting the data and conducting preliminary analyses on all study variables. The following variables were assessed: Posttraumatic Growth Inventory (PTGI) total score and subscale scores; COPE subscale scores for Acceptance coping, Active coping and Denial coping; Barthel Index total score; Multidimensional Scale of Social Support (MSPSS) total score; Rumination Scale total score and subscale scores; Impact of Events Scale Revised (IES-R) total score.

\subsubsection{Missing data}

Forty-seven participants completed all measures in the Stroke Survivors' Survey (see Appendix N) at time 1. However, four participants did not complete any of the measures at time 2. One participant was excluded from the study due to a disclosure of misdiagnosis of stroke which resulted in the participant not meeting the inclusion criteria for the study. Three other participants were not contactable at time 2 and therefore, the measures at time 2 were not completed. The literature recommends that the best practice for longitudinal analysis 
involves using a balanced design whereby all participants have the same number of repeated measurements over time (Fitzmaurice \& Ravichandran, 2008). Therefore, these three participants were also excluded from the study and their data were not retained for further analyses. No other missing data were identified as part of the data screening process.

\subsubsection{Error analysis and outliers}

The minimum and maximum values for each variable were screened to ensure that all data fell within the possible range for each variable. Visual inspection of the data did not reveal any errors in data entry. Outlier analysis was conducted to identify extreme values as such values can introduce bias to subsequent statistical analyses and impact on Type 1 errors as well as power (Keslmann et al. 2008). Visual inspection of the frequency distributions and box plots revealed that there were extreme values for some of the variables.

On inspection, none of the extreme values identified appeared to be due to commonly reported reasons for outliers such as data entry error, intentional or motivated misreporting, sampling error, standardisation failure or faulty distributional assumptions (Osborne, 2013, pp. 144-149). Moreover, the extreme scores identified appeared to be legitimate cases sampled from the target population as the demographic and clinical characteristics of the cases linked to the outliers were similar to the rest of the sample (see section 3.2.3).

In handling these extreme values, it was decided not to remove them from the data set. Bakker and Wicherts (2014) argue that removing outliers from the data set and continuing with parametric analyses impacts on the estimation of standard error. An alternative approach to removing outliers is to apply transformations to the nonnormal data variables (Field, 2013). However, critics of transformations suggest that they do not always result in a normal distribution, they reduce power and can alter the nature of the data which can lead to difficulties with interpretation (Osborne, 2013). Instead, Bakker and Wicherts (2014) recommend using non-parametric or robust statistical methods such as bootstrapping without removing outliers when there is a nonnormal distribution. Given that nonparametric tests are often less powerful than parametric analyses and can still be affected by extreme scores (Osborne, 2013), bootstrapping methods have been recommended as an appropriate 
approach to statistical analysis when legitimate outliers lead to a nonnormal distribution (Wilcox, 2012).

\subsubsection{Distribution of the main study variables}

Skewness and kurtosis values for most of the variables were within the acceptable range of -1.5 and 1.5 (Tabachnick \& Fidell, 2007) (see Appendix P for exceptions). Sharpiro-Wilk's tests, as well as visual inspection of the histograms, normal Q-Q plots and box plots, indicated that most variables were not normally distributed (i.e. $p<.05$ ) with the exception of PTGI at time $1(p=.065)$ and PTGI at time $2(p=.169)$.

Data from psychological measures and clinical populations are often nonnormal (Micceri, 1989). Of note, data from psychological measures are often also highly skewed in non-clinical populations (Rutter \& Miglioretti, 2003). According to Wright et al. 2011, the assumptions for many parametric tests '...often make unrealistic assumptions about variables' distributions...in data derived from clinical samples, or when looking at groups responding at the extreme end of clinical constructs'. The literature also suggests that data are often nonnormal for psychometric reasons: Firstly, the total scores on psychological measures are bounded by the number of scoring options; secondly, psychological measures are typically not very long in research; and thirdly, a measure may capture aspects of a latent trait which does not fit with the study sample (Bakker \& Wicherts, 2014). However, several variables violated the assumptions required for parametric statistical analyses in this study. Based on the best evidence available it was decided that a more robust approach to inferential statistical analysis would be taken by using bootstrapping methods (Efron \& Tibshirani, 1993; see section 3.2.5).

\subsubsection{Other assumptions}

A priori assumptions for hierarchical regression analysis were also tested. The assumption for absence of multicollinearity was met. As Field (2013) recommends, none of the intercorrelations between different predictor variables were above $r=0.80$. The assumption of linearity was also met, following the inspection of scatterplots against each variable. The 
assumption for homoscedasticity was tested by plotting the standardised residuals against the standardised predicted values for each hierarchical regression. On visual inspection, the assumption of homoscedasticity was met. The assumption of independent errors was tested by using the Durbin-Watson statistic to test for independent errors. The Durbin-Watson statistic for each regression ranged from 1.82 to 1.92 and found that the assumption was met (i.e. values close to 2 (Field, 2013)).

\subsubsection{Approach to planned statistical analysis}

Bootstrapping methods (Efron \& Tibshirani, 1993) were considered the best approach to planned analysis given the presence of legitimate outliers, the nonnormal distribution of several variables and the assumption being met that the sample was thought to be representative of the target population (Bakker \& Wicherts, 2014; Aguiin et al. 2013; Wilcox, 2002; see Appendix Q for consultation with statistician).

A bootstrapping module available on SPSS software version 20 (IBM corporation, 2011) was used to conduct the planned statistical analysis (see section 2.6). Bootstrapping involves estimating the properties of the sampling distribution from the sample data and it produces percentile bootstrap confidence intervals based on the values between which $95 \%$ of bootstrap sample estimates fall (Field, 2013). It can be used to find SEs and Cls for almost any statistic (Field, 2013). A bias-corrected and accelerated or BCa method was used to minimise bias of the mean and to produce more accurate confidence intervals than the percentile method (Efron \& Tibshirani, 1993; Field, 2013). The confidence limits generated were used to test the null hypothesis for each hypothesis. If the confidence limits included 0.0, then the null hypothesis was accepted. The bootstrapping module was used for correlational analyses, t-tests, regression analyses and mediation analyses, unless otherwise stated. Bootstrapping enabled inferences to be made on normally distributed data, as well as data where legitimate outliers lead to a nonnormal distribution on a few of the variables (Wright et al. 2011). 


\subsection{Characteristics of the sample}

\subsubsection{Demographic and background characteristics of the sample}

The demographic questionnaire was completed at time 1 and the key characteristics of the sample are summarised in Table 3.1. Of note, the mean number of months post-stroke for participants at time 1 was 4.65 months. Participants were followed up six months after time 1 and the mean number of months post-stroke at time 2 was 10.72 .

Table 3.1 Socio-demographic and background characteristics of the sample.

\begin{tabular}{|c|c|c|}
\hline & & Participants $(\mathrm{N}=43)$ \\
\hline Number of females (\%) & & $18(42)$ \\
\hline Mean age (SD) & & $74.53(9.96)$ \\
\hline Ethnicity, n (\%) & British & $43(100)$ \\
\hline Occupation, n (\%) & $\begin{array}{l}\text { Retired } \\
\text { In employment }\end{array}$ & $\begin{array}{l}38(88) \\
5(12)\end{array}$ \\
\hline Mean months since stroke (SD) & $\begin{array}{l}\text { Time } 1 \\
\text { Time } 2\end{array}$ & $\begin{array}{l}4.65(1.65) \\
10.72(1.71)\end{array}$ \\
\hline Type of stroke, $n(\%)$ & $\begin{array}{l}\text { Ischaemic } \\
\text { Haemorrhagic }\end{array}$ & $\begin{array}{l}38(88) \\
5(12)\end{array}$ \\
\hline Thrombolysed, $\mathrm{n}(\%)$ & $\begin{array}{l}\text { Yes } \\
\text { No }\end{array}$ & $\begin{array}{l}3(7) \\
40(93)\end{array}$ \\
\hline Communication difficulties, $\mathrm{n}(\%)$ & $\begin{array}{l}\text { Not at all } \\
\text { Mildly } \\
\text { Severely }\end{array}$ & $\begin{array}{l}28(65) \\
10(23) \\
5(12)\end{array}$ \\
\hline Memory difficulties, n (\%) & $\begin{array}{l}\text { Not at all } \\
\text { Mildly } \\
\text { Severely }\end{array}$ & $\begin{array}{l}26(60) \\
11(26) \\
6(14)\end{array}$ \\
\hline Change in relationships & $\begin{array}{l}\text { Not at all } \\
\text { Somewhat } \\
\text { Most definitely }\end{array}$ & $\begin{array}{l}21(49) \\
18(42) \\
4(9)\end{array}$ \\
\hline Current feelings of depression & $\begin{array}{l}\text { Never } \\
\text { Sometimes } \\
\text { Often }\end{array}$ & $\begin{array}{l}19(44) \\
18(42) \\
6(14)\end{array}$ \\
\hline Current feelings of anxiety & $\begin{array}{l}\text { Never } \\
\text { Sometimes } \\
\text { Often }\end{array}$ & $\begin{array}{l}22(51) \\
17(40) \\
4(9)\end{array}$ \\
\hline Treatment for anxiety/depression & $\begin{array}{l}\text { Yes } \\
\text { No }\end{array}$ & $\begin{array}{l}6(14) \\
37(86)\end{array}$ \\
\hline Change in sleep & $\begin{array}{l}\text { Not at all } \\
\text { Somewhat } \\
\text { Most definitely }\end{array}$ & $\begin{array}{l}30(70) \\
9(21) \\
4(9)\end{array}$ \\
\hline
\end{tabular}




\subsubsection{Response rate}

Of the 58 participants who were sent information about the study, $81 \%$ agreed to participate in the study when contacted by telephone. Given that $93 \%$ of the participants recruited at Time 1 completed follow-up, the remaining sample is thought to be representative of the original target population $(n=43)$. One participant was excluded from the study due to a disclosure disconfirming their stroke diagnosis. The 3 other participants who did not complete follow up were not contactable at the time of follow-up.

\subsubsection{Clinical characteristics of the sample}

Means, standard deviations, and ranges for all the main study variables at time 1 and time 2 are summarised in Table 3.2. Paired samples t-test were conducted to determine whether the difference between each variable at time 1 and time 2 was significant and the results are also summarised in Table 3.2. Bonferroni adjustments were not computed because the number of other paired samples t-tests was not seen as relevant for interpreting the differences between each individual variable at time 1 and 2 (see section 2.6.4). Perneger (1998) recommends describing what tests have been performed and why as the best way of dealing with multiple comparisons. As such, the paired samples t-tests reported in Table 3.2 were performed to test whether the difference between each variable at time 1 and 2 was significant. Bivariate correlations between the main study variables are summarised in Table 3.3 and correlations were performed to test several of the key hypotheses of this study. More detailed analyses of the hypothesised differences between the main study variables can be found in sections 3.4, 3.5, 3.6 and 3.7.

All variables were significantly different between time 1 and time 2 apart from rumination $(t(43)=1.07, p=0.29)$, daily functioning $(t(43)=1.99, p=0.051)$ and posttraumatic stress symptoms $(\mathrm{t}(43)=-1.22, \mathrm{p}=0.23$ ) (see Table 3.2 ). This suggests that perhaps rumination, daily functioning and PTSS remained relatively stable between time 1 and time 2 . 
Table 3.2 Descriptive statistics for the key variables over time.

\begin{tabular}{|l|lll|lll|l|}
\hline & \multicolumn{7}{|c}{ Participants (N=43) } \\
\cline { 2 - 8 } & Time 1 & & & Time 2 & \\
\cline { 2 - 8 } & Mean & SD & Range & Mean & SD & Range & Sig. \\
\hline PTGI T & 35.14 & 26.22 & $0-97$ & 52.14 & 25.93 & $0-102$ & $\mathrm{t}(43)=11.65, \mathrm{p}<.001$ \\
New possibilities & 4.77 & 6.29 & $0-22$ & 8.35 & 7.52 & $0-30$ & $\mathrm{t}(43)=6.76, \mathrm{p}<.001$ \\
Relating to others & 15.65 & 10.41 & $0-35$ & 21.67 & 11.46 & $0-54$ & $\mathrm{t}(43)=7.585, \mathrm{p}<.001$ \\
Personal strength & 6.65 & 5.97 & $0-19$ & 10.72 & 5.68 & $0-20$ & $\mathrm{t}(43)=8.15, \mathrm{p}<.001$ \\
Spiritual change & 1.49 & 3.23 & $0-10$ & 1.91 & 3.57 & $0-10$ & $\mathrm{t}(43)=2.22, \mathrm{p}<.05$ \\
Appreciation of life & 6.53 & 4.42 & $0-15$ & 10.88 & 3.98 & $0-16$ & $\mathrm{t}(43)=8.22, \mathrm{p}<.001$ \\
\hline COPE scale & & & & & & & \\
Active coping & 10.84 & 3.61 & $4-16$ & 13.51 & 3.20 & $4-16$ & $\mathrm{t}(43)=7.19, \mathrm{p}<.001$ \\
Acceptance coping & 13.16 & 3.65 & $4-16$ & 14.6 & 2.42 & $4-16$ & $\mathrm{t}(43)=3.16, \mathrm{p}<.005$ \\
Denial coping & 6.05 & 2.52 & $4-13$ & 4.4 & 0.90 & $4-8$ & $\mathrm{t}(43)=-3.88, \mathrm{p}<.001$ \\
\hline Rumination T & 25.35 & 10.73 & $14-46$ & 26.12 & 10.349 & $14-48$ & $\mathrm{t}(43)=1.07, \mathrm{p}=0.289$ \\
Soon after stroke & 13.19 & 5.5 & $7-25$ & 12.95 & 6.047 & $7-27$ & $\mathrm{t}(43)=1.95, \mathrm{p}=0.35$ \\
Recent & 12.26 & 5.61 & $7-23$ & 13.33 & 5.375 & $7-23$ & $\mathrm{t}(43)=1.58, \mathrm{p}=.12$ \\
\hline Barthel T & 18.19 & 3.18 & $7-20$ & 18.72 & 2.930 & $9-20$ & $\mathrm{t}(43)=1.99, \mathrm{p}=.051$ \\
\hline IES-R T & 3.51 & 10.93 & $0-70$ & 1.74 & 3.381 & $0-13$ & $\mathrm{t}(43)=-1.22, \mathrm{p}=0.230$ \\
\hline MSPSS T & 64.42 & 8.27 & $46-72$ & 67.16 & 6.955 & $44-72$ & $\mathrm{t}(43)=4.34, \mathrm{p}<.001$ \\
\hline
\end{tabular}

( $\mathrm{T}$ = total scale score; PTGI = Posttraumatic Growth Inventory; IES-R =Impact of Events Scale revised; MSPSS = Multidimensional scale of social support)

a Bias corrected confidence intervals were computed based on 2,000 bootstrap samples but are not reported due to limited space. The difference between T1 and T2 was significant $(p<.05)$ when its bootstrapped confidence intervals did not cross zero 
Table 3.3 Bivariate correlations between the main study variable.

\begin{tabular}{|c|c|c|c|c|c|c|c|c|c|c|c|c|c|c|c|c|c|c|}
\hline & & Variable & Age & 1 & 2 & 3 & 4 & 5 & 6 & 7 & 8 & 9 & 10 & 11 & 12 & 13 & 14 & 15 \\
\hline \multirow{15}{*}{$\begin{array}{l}\stackrel{-}{\tilde{g}} \\
\stackrel{\underline{E}}{=}\end{array}$} & 1 & PTGI total & -0.29 & - & & & & & & & & & & & & & & \\
\hline & 2 & New possibilities & $-0.40 *$ & $0.81 *$ & - & & & & & & & & & & & & & \\
\hline & 3 & Relating to others & -0.26 & $0.93^{*}$ & $0.62^{*}$ & - & & & & & & & & & & & & \\
\hline & 4 & Personal strength & -0.19 & 0.91* & $0.69 *$ & $0.84 *$ & - & & & & & & & & & & & \\
\hline & 5 & Spiritual change & -0.03 & $0.61 *$ & $0.52 *$ & $0.48 *$ & $0.50 *$ & - & & & & & & & & & & \\
\hline & 6 & Appreciation of life & -0.29 & $0.81 *$ & $0.59 *$ & $0.77 *$ & $0.65^{*}$ & $0.32 *$ & - & & & & & & & & & \\
\hline & 7 & Active coping & 0.02 & $0.39 *$ & $0.44^{*}$ & $0.32 *$ & $0.33^{*}$ & 0.10 & 0.29 & - & & & & & & & & \\
\hline & 8 & Acceptance coping & 0.15 & 0.10 & 0.12 & 0.57 & 0.14 & 0.13 & 0.01 & $0.58 *$ & - & & & & & & & \\
\hline & 9 & Denial coping & -0.30 & 0.22 & 0.11 & 0.27 & 0.14 & -0.15 & 0.28 & 0.12 & -0.07 & - & & & & & & \\
\hline & 10 & Rumination total & $-0.35^{*}$ & $0.63^{*}$ & $0.52 *$ & $0.58 *$ & $0.53 *$ & 0.21 & $0.67 *$ & 0.31 & -0.08 & $0.50 *$ & - & & & & & \\
\hline & 11 & Soon after stroke & $-0.32 *$ & $0.63^{*}$ & $0.54 *$ & $0.58^{*}$ & $0.56^{*}$ & 0.23 & $0.63^{*}$ & 0.28 & -0.05 & $0.43^{*}$ & $0.97^{*}$ & - & & & & \\
\hline & 12 & Recent & -0.34 & $0.60 *$ & $0.48 *$ & $0.56 *$ & $0.48^{*}$ & 0.21 & $0.67 *$ & 0.34 & -0.10 & $0.53^{*}$ & $0.97^{*}$ & $0.89 *$ & - & & & \\
\hline & 13 & Barthel Index & -0.21 & 0.13 & 0.15 & 0.12 & 0.11 & -0.65 & 0.08 & -0.02 & 0.07 & -0.01 & -0.03 & -0.04 & -0.08 & - & & \\
\hline & 14 & IES-R & -0.22 & 0.02 & 0.02 & 0.06 & -0.04 & -0.04 & 0.04 & 0.04 & -0.12 & 0.43 & $0.40 *$ & $0.37 *$ & 0.39* & -0.16 & - & \\
\hline & 15 & MSPSS & -0.18 & 0.26 & 0.15 & $0.30 *$ & 0.23 & -0.00 & 0.22 & 0.10 & 0.03 & 0.09 & 0.09 & 0.13 & 0.13 & -0.00 & -0.25 & - \\
\hline \multirow{15}{*}{$\underset{\stackrel{N}{\varrho}}{\underline{E}}$} & 1 & PTGI total & $-0.36 *$ & - & & & & & & & & & & & & & & \\
\hline & 2 & New possibilities & $-0.41 *$ & $0.85^{*}$ & - & & & & & & & & & & & & & \\
\hline & 3 & Relating to others & -0.26 & $0.89 *$ & $0.65^{*}$ & - & & & & & & & & & & & & \\
\hline & 4 & Personal strength & -0.14 & 0.84* & 0.63* & 0.81* & - & & & & & & & & & & & \\
\hline & 5 & Spiritual change & -0.07 & $0.60 *$ & $0.48 *$ & $0.48 *$ & $0.42 *$ & - & & & & & & & & & & \\
\hline & 6 & Appreciation of life & $-0.39 *$ & $0.67 *$ & $0.60 *$ & $0.60 *$ & $0.49 *$ & $0.31 *$ & - & & & & & & & & & \\
\hline & 7 & Active coping & 0.02 & $0.44 *$ & $0.37 *$ & $0.38 *$ & $0.41 *$ & 0.07 & $0.51 *$ & - & & & & & & & & \\
\hline & 8 & Acceptance coping & 0.12 & 0.21 & 0.26 & 0.13 & 0.16 & 0.16 & 0.11 & 0.48 & - & & & & & & & \\
\hline & 9 & Denial coping & -0.07 & -0.11 & -0.20 & -0.02 & 0.04 & -0.18 & -0.32 & -0.18 & -0.09 & - & & & & & & \\
\hline & 10 & Rumination total & -0.28 & $0.63^{*}$ & $0.52 *$ & $0.63^{*}$ & $0.52 *$ & 0.27 & $0.53^{*}$ & 0.15 & -0.16 & -0.01 & - & & & & & \\
\hline & 11 & Soon after stroke & -0.24 & $0.62 *$ & $0.48 *$ & $0.65 *$ & $0.54 *$ & $0.33^{*}$ & $0.48^{*}$ & 0.15 & -0.06 & 0.07 & $0.91 *$ & - & & & & \\
\hline & 12 & Recent & -0.28 & 0.51* & $0.47^{*}$ & $0.50 *$ & $0.42 *$ & 0.16 & $0.50 *$ & 0.12 & -0.22 & -0.10 & $0.89 *$ & $0.64 *$ & - & & & \\
\hline & 13 & Barthel Index & -0.14 & 0.25 & $0.32 *$ & 0.14 & 0.16 & 0.14 & 0.20 & 0.339 & 0.29 & -0.29 & -0.10 & 0.04 & -0.23 & - & & \\
\hline & 14 & IES-R & 0.06 & -0.00 & -0.06 & 0.06 & 0.01 & -0.08 & 0.09 & .06 & $-0.33^{*}$ & -0.16 & $0.34 *$ & 0.19 & $0.43^{*}$ & -0.12 & - & \\
\hline & 15 & MSPSS & -0.28 & $0.41 *$ & $0.37^{*}$ & $0.41 *$ & 0.29 & 0.20 & $0.49 *$ & .17 & 0.17 & -0.12 & 0.16 & 0.12 & 0.19 & -0.02 & -0.29 & \\
\hline
\end{tabular}

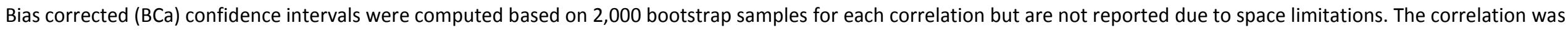
significant $\left({ }^{*}=p<.05\right)$ when its bootstrapped confidence intervals did not cross zero. 


\subsubsection{Relationship between demographic and clinical characteristics of the sample}

Prior to inferential analysis, the relationships between key socio-demographic variables like age, gender and occupation and the main study variables were investigated to identify potential confounding variables. Pearson's correlation coefficients were calculated to determine the relationship between age and the main study variables. Age was negatively correlated with rumination total at time $1(p=-0.35)$ and PTGI total at time $2(p=-0.36)$. Age was also negatively correlated with the New Possibilities subscale of the PTGI at time 1 and 2 ( $p=-0.4$ and $p=-0.36$ respectively). However, given that age did not correlate with predictor variables and PTG, age was considered a 'third' variable related to the development of PTG (Kamangar, 2012). According to Schaefer and Moos' (1996) model of PTG, age is hypothesised as predictive of PTG. Therefore, age was considered as a predictor variable in hierarchical regression analysis (see section 3.7.3).

Independent t-tests did not identify any significant differences between gender and the main study variables $(p>0.5)$. Analysis of variance (ANOVA) was used to compare scores on the main variables in those who were retired, in employment or unemployed. No significant group effects were found.

\subsection{Characteristics of PTG}

\subsubsection{Occurrence of PTG over time}

Descriptive statistics were used to determine whether participants experienced PTG at two different time points post stroke. The mean PTGI total scale score at time 1 was 35.14 (SD = 26.22 , range $=0-97)$ and the mean at time 2 was 52.14 (SD $=25.93$, range $=0-102)$ indicate that PTG occurred at both time points (i.e. PTGI total score $>0$ ). A paired sample t-test indicated that participants experienced significantly more PTG, as measured by the PTGI, at time 2 compared to time $1(t(43)=11.65, p<0.001)$. 


\subsubsection{Subscale analyses}

PTGI subscale scores were computed to determine whether PTGI scores changed between time 1 and 2. Paired sample t-tests indicated that each of the five subscales of the PTGI was significantly higher at time 2 compared to time 1 (see Table 3.2).

\subsubsection{Comparison with previously reported data}

The mean PTGI total scores in this study were compared with findings published in similar studies. T-tests were calculated by hand and found that none of the mean PTGI scores cited in similar stroke research differed significantly from the means observed in this study $(t(103)=$ 0.3596, $\mathrm{p}>.05$ (Gangstad et al. 2009); $\mathrm{t}(85)=-0.14, \mathrm{p}>.05$ (Kuenemund et al. 2014); $t(115)=1.1935, p>$.05) (see Appendix R for formula used).

Table 3.4 Comparison of PTGI in the current study with previously reported data.

\begin{tabular}{|c|c|c|c|c|c|c|c|c|c|c|c|}
\hline \multirow[b]{3}{*}{ Study } & \multirow[b]{3}{*}{ Population } & \multirow[b]{3}{*}{ Country } & \multirow[b]{3}{*}{$\mathbf{N}$} & \multirow[b]{3}{*}{ Mean Age } & \multicolumn{7}{|l|}{ PTGI } \\
\hline & & & & & \multicolumn{2}{|c|}{ Total Score } & \multicolumn{5}{|c|}{ Subscales - mean (SD) } \\
\hline & & & & & Mean & SD & NP & RTO & PS & SC & AOL \\
\hline $\begin{array}{l}\text { Gangstad } \\
\text { et al. (2009) }\end{array}$ & $\begin{array}{l}\text { Stroke } \\
\text { survivors }\end{array}$ & UK & 60 & 71.67 & 50.93 & 19.92 & - & - & - & - & - \\
\hline $\begin{array}{l}\text { Kuenemund } \\
\text { et al. (2014) }\end{array}$ & $\begin{array}{l}\text { Stroke } \\
\text { survivors }\end{array}$ & Germany & 42 & 52.83 & 57.69 & 19.28 & $\begin{array}{l}2.58 \\
(1.12)\end{array}$ & $\begin{array}{l}2.99 \\
(1.10)\end{array}$ & $\begin{array}{l}2.36 \\
(0.99)\end{array}$ & $\begin{array}{l}2.06 \\
(1.13)\end{array}$ & $\begin{array}{l}3.44 \\
(1.13)\end{array}$ \\
\hline Weiss (2004) & $\begin{array}{l}\text { Cancer } \\
\text { survivors }\end{array}$ & US & 72 & 54.2 & 57.9 & 24.5 & $\begin{array}{l}10.6 \\
(6.9)\end{array}$ & $\begin{array}{l}19.6 \\
(9.3)\end{array}$ & $\begin{array}{l}12.0 \\
(6)\end{array}$ & $\begin{array}{l}4.3 \\
(3.2)\end{array}$ & $\begin{array}{l}11.1 \\
(3.6)\end{array}$ \\
\hline $\begin{array}{l}\text { Bluvstein } \\
\text { et al. (2012) }\end{array}$ & MI survivors & Israel & 82 & 63.7 & 41.3 & - & - & - & - & - & - \\
\hline
\end{tabular}




\subsection{Social support, coping, daily functioning and posttraumatic growth.}

This study hypothesised that PTG at time 2 would be positively associated with social support, active coping, acceptance coping, daily functioning and negatively associated with denial coping at time 1 and time 2 . It was predicted that social support would be positively associated with rumination, active coping, daily functioning, acceptance coping and negatively associated with denial coping. It was also predicted that active coping would mediate the relationship between social support and PTGI at time 2. These hypotheses were tested, in the first instance, by examining the bivariate correlations relevant to the hypotheses outlined. Mediation analysis was then conducted to determine whether the relationship between social support and PTG was mediated by active coping.

\subsubsection{Correlational analyses}

Table 3.3 shows the inter-correlations for all the variables between time 1 and time 2 . Correlational analysis revealed that PTG at time 2 was positively associated with active coping at time $2\left(p_{b}=0.44, \mathrm{BCa} \mathrm{Cl}[0.21,0.62], p=.003\right)$ and social support at time $2\left(p_{b}=0.41, B C a\right.$ $\mathrm{Cl}[0.16,0.60], \mathrm{p}=0.006)$. Active coping at time 1 was positively associated with PTGI at time $2\left(p_{b}=0.48, \mathrm{BCa} \mathrm{Cl}[0.23,0.66], p=0.001\right)$. Although social support at time 1 was not associated with the PTGI total score at time 2, a significant association was found between social support at time 1 and the PTGI subscale of 'relating to others' was found ( $p_{b}=0.30, B C a$ $\mathrm{Cl}[0.02,0.54], \mathrm{p}=0.045)$. However, social support at time 2 was significantly associated with the PTG at time $2\left(p_{b}=0.41, \mathrm{BCa} C l[0.21,0.62], p=0.003\right)$. Acceptance coping was not significantly associated with PTG at any time point. Of note, daily functioning at time 2 was positively associated with the PTGI subscale of 'new possibilities' at time $2\left(\mathrm{p}_{\mathrm{b}}=0.32, \mathrm{BCa} \mathrm{Cl}\right.$ $[0.14,0.48], p=0.035)$. Social support was not significantly correlated with rumination, any type of coping, or daily functioning. 
Table 3.5 Summary of key findings relating to coping, social support, functioning and PTG.

\begin{tabular}{|ll|c|c|}
\hline Hypotheses: & & Time & Sig. \\
\hline PTGI at T2 will be positively associated with: & Social Support & T1 & $\mathrm{ns}$ \\
& Social Support & $\mathrm{T} 2$ & $\mathrm{r}=0.41$ \\
& Active Coping & $\mathrm{T} 1$ & $\mathrm{r}=0.48$ \\
& Active Coping & $\mathrm{T} 2$ & $\mathrm{r}=0.44$ \\
& Acceptance Coping & $\mathrm{T} 1 \& \mathrm{~T} 2$ & $\mathrm{~ns}$ \\
& Daily functioning & $\mathrm{T} 1 \& \mathrm{~T} 2$ & $\mathrm{~ns}$ \\
\hline PTGI at T1 will be negatively associated with: & Denial coping & $\mathrm{T} 1$ & $\mathrm{~ns}$ \\
\hline PTGI at T2 will be negatively associated with: & Denial coping & $\mathrm{T} 2$ & $\mathrm{~ns}$ \\
\hline Social support will be positively associated with: & Active coping & T1 \& T2 & $\mathrm{ns}$ \\
& Acceptance coping & $\mathrm{T} 1 \& \mathrm{~T} 2$ & $\mathrm{~ns}$ \\
& Daily functioning & $\mathrm{T} 1 \& \mathrm{~T} 2$ & $\mathrm{~ns}$ \\
\hline Social support will be negatively associated with: & Denial coping & T1 \& T2 & $\mathrm{ns}$ \\
\hline Active coping will mediate the effect of social support on PTGl at T2 & T1 \& T2 & $\mathrm{ns}$ \\
\hline Acceptance coping will mediate the effect of social support on PTGl at T2 & T1 \& T2 & $\mathrm{ns}$ \\
\hline
\end{tabular}

$\mathbf{T 1}=$ time 1; $\mathbf{T 2}$ = time 2; $\mathbf{n s}=$ not significant.

\subsubsection{Mediation analyses}

Mediation analyses were conducted to determine whether the relationship between social support and PTG was mediated by active coping. This analysis was carried out using PROCESS software (Hayes, 2014) (see section 2.6 .2 for further information). Active coping and acceptance coping were considered as possible mediators of the relationship between social support and PTG at time 2. Simple non-parametric bootstrapping tests were conducted in order to test the significance of all hypothesised mediators. In this way, each hypothesised mediator was tested individually using separate mediation analysis. Mediators were interpreted as significant $(p<.05)$ when bootstrapped 95\% confidence intervals for that variable did not cross zero (Hayes, 2013). As Table 3.6 shows, neither active coping nor acceptance coping were found to mediate the relationship between social support at time 1 or time 2, and PTG at time 2. 
Table 3.6 Simple mediation model of the indirect effects of social support on PTG through active coping and acceptance coping.

\begin{tabular}{|c|c|c|c|c|c|}
\hline \multirow{2}{*}{$\begin{array}{l}\text { Independent } \\
\text { Variable }\end{array}$} & \multirow[t]{2}{*}{ Mediator } & \multirow{2}{*}{$\begin{array}{l}\text { Unstandardised } \\
\text { Coefficient }^{\mathrm{a}}\end{array}$} & \multicolumn{2}{|c|}{ Bootstrapped $95 \% \mathrm{Cl}^{\mathrm{a}}$} & \multirow[t]{2}{*}{ Model $R^{2}$} \\
\hline & & & Lower & Upper & \\
\hline \multirow{4}{*}{$\begin{array}{l}\text { Social support } \\
\text { T1 }\end{array}$} & ActCope T1 & 0.01 & -0.5 & 0.26 & 0.001 \\
\hline & ActCopeT2 & 0.06 & -0.14 & 0.75 & 0.019 \\
\hline & AccCopeT1 & 0.02 & -0.6 & 0.4 & 0.012 \\
\hline & AccCope T2 & 0.07 & -0.15 & 1.12 & 0.031 \\
\hline \multirow{4}{*}{$\begin{array}{l}\text { Social support } \\
\text { T2 }\end{array}$} & ActCope T1 & 0.11 & -0.13 & 0.94 & 0.011 \\
\hline & ActCopeT2 & 0.07 & -0.07 & 0.59 & 0.025 \\
\hline & AccCopeT1 & 0.17 & -0.2 & 1.27 & 0.02 \\
\hline & AccCope T2 & 0.08 & -0.11 & 0.85 & 0.03 \\
\hline
\end{tabular}

a Bias corrected confidence intervals were computed based on 2,000 bootstrap samples. Mediator was significant $(p<.05)$ when its bootstrapped confidence intervals did not cross zero.

\subsection{Rumination, coping, social support, posttraumatic stress and PTG}

This study hypothesised that rumination and posttraumatic stress symptoms (PTSS) would be positively associated with PTGI at time 1 and 2. It was predicted that rumination at time 1 would be positively associated with acceptance coping at time 2 and negatively associated with denial coping at time 2. It was also predicted that acceptance coping at time 2 would be negatively associated with PTSS at time 2 . In addition, it was predicted that rumination would be associated with PTSS at time 1 and 2. These hypotheses were tested, in the first instance, by examining the bivariate correlations relevant to the hypotheses outlined. Mediation analysis was then performed to determine whether the relationship between PTSS and PTG was mediated by rumination.

\subsubsection{Correlational analyses}

Correlational analysis revealed that rumination at time 1 was positively associated with PTGI at time $2\left(\left(\mathrm{p}_{\mathrm{b}}=0.62, \mathrm{BCa} \mathrm{Cl}[0.41,0.80], \mathrm{p}=0.000\right)\right.$. Rumination at time 1 was correlated with all PTGI subscales apart from 'spiritual change' at time 2 (see Table 3.3). Rumination at time 
1 was positively associated with PTGI at time $1\left(\left(\mathrm{p}_{\mathrm{b}}=0.63, \mathrm{BCa} \mathrm{Cl}[0.40,0.83], \mathrm{p}=0.000\right)\right.$ and rumination at time 2 was positively associated with PTGI at time $2\left(\left(\mathrm{p}_{\mathrm{b}}=0.63, \mathrm{BCa} \mathrm{Cl}[0.44\right.\right.$, 0.77], $p=0.000$ ). PTSS was not significantly correlated with PTGI at time 1 or 2 . Rumination at time 1 was not significantly associated with acceptance coping at time 2 . Nor was rumination negatively associated with denial coping at time 1 or 2 . Rather, denial coping at time 1 was positively associated with rumination at time $2\left(p_{b}=0.32, \mathrm{BCa} C l[0.02,0.58], p=\right.$ 0.032). Interestingly, denial coping at time 1 was also associated with the PTGI subscale 'appreciation of life' at time $2\left(p_{b}=0.39, \mathrm{BCa} C l[0.10,0.60], p=0.010\right)$.

Rumination at time 1 was positively associated with PTSS at time $1\left(p_{b}=0.40, \mathrm{BCa} \mathrm{Cl}[0.17\right.$, $0.71], p=0.008)$. Similarly, rumination at time 2 was associated with PTSS at time $2\left(p_{b}=0.34\right.$, $\mathrm{BCa} \mathrm{Cl}[0.13,0.55], \mathrm{p}=0.026)$. Of note, PTSS at time 2 was significantly associated with the rumination subscale for 'recent' rumination at time $2\left(p_{b}=0.43, \mathrm{BCa} \mathrm{Cl}[0.23,0.62], p=0.004\right)$ but was not significantly associated with 'soon after the event' rumination at time 2 . This suggests that participants who experienced posttraumatic stress symptoms at time 2 were more likely to experience recent rumination as opposed to rumination soon after the event.

Table 3.7 Summary of key findings relating to rumination, coping, PTSS and PTG.

\begin{tabular}{|c|c|c|c|}
\hline \multicolumn{2}{|l|}{ Hypotheses } & \multirow{2}{*}{$\begin{array}{r}\text { Time } \\
\mathrm{T} 1\end{array}$} & \multirow{2}{*}{$\begin{array}{l}\text { Sig. } \\
0.63\end{array}$} \\
\hline PTGI at T1 will be positively associated with: & Rumination & & \\
\hline & PTSS & $\mathrm{T} 1$ & ns \\
\hline \multirow[t]{3}{*}{ PTGI at T2 will be positively associated with: } & Rumination & T1 & 0.62 \\
\hline & Rumination & $\mathrm{T} 2$ & 0.63 \\
\hline & PTSS & $\mathrm{T} 2$ & ns \\
\hline Rumination at T1 will be positively associated with: & PTSS & $\mathrm{T} 1$ & 0.40 \\
\hline \multirow[t]{2}{*}{ Rumination at $\mathrm{T} 2$ will be positively associated with: } & PTSS & T2 & 0.37 \\
\hline & Acc. Coping & $\mathrm{T} 2$ & ns \\
\hline Rumination at T1 will be negatively associated with: & Den. Coping & T1 & ns \\
\hline Rumination at $\mathrm{T} 2$ will be negatively associated with: & Den. Coping & $\mathrm{T} 2$ & ns \\
\hline Denial at T1 will be positively associated with: & Rumination & T2 & 0.32 \\
\hline Acceptance coping at time 2 will be negatively associated with: & PTSS & T2 & -0.33 \\
\hline Social support will be positively associated with: & Rumination & T1\&T2 & ns \\
\hline Rumination will mediate the effect of PTSS at T1 on PTG at T1 & & T1 & $<0.05$ \\
\hline Rumination will mediate the effect of PTSS at T1 on PTG at T2 & & T1 & $<0.05$ \\
\hline Rumination will mediate the effect of PTSS at T2 on PTG at T2 & & T2 & $<0.05$ \\
\hline
\end{tabular}




\subsubsection{Mediation analysis}

Mediation analysis were conducted to determine whether the relationship between PTSS and PTG was mediated by rumination. This analysis was carried out using PROCESS software (Hayes, 2014) (see section 2.6.2 for further information). Simple non-parametric bootstrapping tests were conducted in order to test the significance of all hypothesised mediators. In this way, each hypothesised mediator was tested individually using separate mediation analysis. Table 3.8 outlines the results of the mediation analysis, whereby mediators were interpreted as significant $(p<.05)$ when bootstrapped $95 \%$ confidence intervals for that variable did not cross zero (Hayes, 2013). As table 3.8 shows, rumination mediated the relationship between PTSS and PTG over time $(p<.05)$. However, rumination did not mediate the relationship between social support and PTG $(p>05)$. Figure 3.1 illustrates the significant mediating relationships outlined in Table 3.8.

Table 3.8 Simple mediation model of the indirect effects of PTSS on PTG at time 2 through rumination

\begin{tabular}{|l|l|l|l|l|l|}
\hline \multirow{2}{*}{ Variable } & Mediator & \multirow{2}{*}{$\begin{array}{l}\text { Unstandardised } \\
\text { Coefficient }\end{array}$} & \multicolumn{2}{|l|}{ Bootstrapped 95\% $\mathrm{Cl}^{\mathrm{a}}$} & \multirow{2}{*}{${\text { Model } \mathbf{R}^{2}}^{\mathrm{a}}$} \\
\cline { 3 - 6 } & & & Lower & Upper & \\
\hline PTSS T1 & Rum T1 & 0.71 & 0.37 & 2.92 & 0.16 \\
\cline { 2 - 6 } & Rum T2 & 0.68 & 0.35 & 2.74 & 0.16 \\
\hline PTSS T2 & Rum T2 & 1.8 & 0.78 & 3.8 & 0.12 \\
\hline Social Support & Rum T1 & 0.18 & -0.39 & 1.01 & 0.01 \\
\hline
\end{tabular}

Codes $^{\text {a }}$ Bias corrected confidence intervals were based on 2,000 bootstrap samples. Mediator was significant ( $p$

$<.05)$ when its bootstrapped confidence intervals did not cross zero 


\section{Rumination}

T1

$$
\mathrm{B}=0.39, \mathrm{p}=.000 \quad \mathrm{~B}=1.81, \mathrm{p}=.000
$$

\section{PTSS T1 $\quad \longrightarrow$ PTGI T1}

$$
\text { Direct effect } B=-0.66, p=.03
$$

Indirect effect $\mathrm{B}=0.71,95 \% \mathrm{Cl}[0.35,2.85]$

\section{Rumination}

$B=0.39, p=.007$
$B=1.73, p=.000$

$B=1.03, p=.025$

Rumination

$\mathrm{T} 2$

\section{PTGI T2}

Direct effect $B=-0.52, p=0.09$

Indirect effect $\mathrm{B}=0.68,95 \% \mathrm{Cl}[0.34,2.74]$
PTSS T2 $\longleftrightarrow$ PTGIT2

Direct effect $B=-1.9, p=.05$

Indirect effect $\mathrm{B}=1.8,95 \% \mathrm{Cl}[0.77,3.93]$

Figure 3.1 Mediation model of the indirect effect of PTSS at time 2 on the relationship between rumination at time 2 and PTGI at time 2 .

\subsection{Predictive utility of models of PTG in this sample}

Hierarchical regression analysis was used to determine which predictors at time 1 were most predictive of PTG at time 2. The predictive utility of two models of PTG was assessed (i.e. Schaerfer \& Moos, 1992; Tedeschi \& Calhoun, 1995, 2004). Each regression was performed using the enter method and the data were bootstrapped. An exploratory model of PTG was also generated using stepwise linear regression.

\subsubsection{Tedeschi and Calhoun (2004) model of PTG and this sample}

The key predictors of PTG that have been identified by Tedeschi and Calhoun (2004) are: rumination, social support, acceptance coping and daily functioning. Each of these variables were entered into the model in blocks using the enter method and factors were entered 
according to the strength of their correlation with PTG (see Table 3.11). With the exception of rumination $(F(1,42)=25.74, p<.001)$, none of the other variables significantly contributed to the proposed model. Rumination accounted for $37 \%$ of the variance in total PTGI at time 2 and was the only significant predictor of PTG. Collectively, the four predictors accounted for $43 \%$ of the variance in PTG.

Table 3.11 Summary of hierarchical regression for Tedeschi and Calhoun (2004) model of PTG

\begin{tabular}{|c|c|c|c|c|c|}
\hline & Adjusted $\mathrm{R}^{2}$ & $\mathbf{F}$ & B & SE $\boldsymbol{\beta}$ & $\beta$ \\
\hline $\begin{array}{l}\text { Model } 1 \\
\text { RuminationTotal T1 }\end{array}$ & 0.37 & $25.742 *$ & 1.52 & 0.621 & $0.62 *$ \\
\hline $\begin{array}{l}\text { Model } 2 \\
\text { RuminationTotal T1 } \\
\text { Social Support T1 }\end{array}$ & 0.39 & $14.485^{*}$ & $\begin{array}{l}1.478 \\
0.591\end{array}$ & $\begin{array}{l}0.296 \\
0.384\end{array}$ & $\begin{array}{l}0.604^{*} \\
0.186\end{array}$ \\
\hline $\begin{array}{l}\text { Model } 3 \\
\text { RuminationTotal T1 } \\
\text { Social Support T1 } \\
\text { Acceptance Coping T1 }\end{array}$ & 0.42 & $11.284^{*}$ & $\begin{array}{l}1.525 \\
0.563 \\
1.43\end{array}$ & $\begin{array}{l}0.289 \\
0.374 \\
0.787\end{array}$ & $\begin{array}{l}0.623 * \\
0.177 \\
0.212\end{array}$ \\
\hline $\begin{array}{l}\text { Model } 4 \\
\text { RuminationTotal T1 } \\
\text { Social Support T1 } \\
\text { Acceptance Coping T1 } \\
\text { Daily functioning T1 }\end{array}$ & 0.43 & $9.018^{*}$ & $\begin{array}{l}1.535 \\
0.565 \\
1.351 \\
1.237\end{array}$ & $\begin{array}{l}0.287 \\
0.371 \\
0.783 \\
0.962\end{array}$ & $\begin{array}{l}0.627^{*} \\
0.178 \\
0.202 \\
0.150\end{array}$ \\
\hline
\end{tabular}

* $p<.05 ;$ Std Error $B=$ standard error of $B ; \beta=$ standardized beta.

\subsubsection{Schaefer and Moos (1998) model of PTG and this sample}

The key predictors of PTG that have been identified by Schaefer and Moos (1992) are: social support, active coping, daily functioning and age. Each of these variables were entered into the model in blocks according to the strength of their correlation with PTG using the enter method. Only active coping and age significantly added to the model (see Table 3.12). Active coping was entered into the model first and contributed significantly to the model accounting for $21 \%$ of the variation PTGI at time $2(F(1,42)=12.26, p<.001)$. Adding age to the regression model explained an additional 13\% of the variance in PTG at time 2 and this predictor also significantly contributed to the model $(F(2,42)=11.99, p<.001)$. When all four predictors were entered in the model, neither social support nor daily functioning were significant predictors 
of PTG at time 2. The final model accounted for $33 \%$ of the variance in PTG scores $\left(F_{4,42}=6.23\right.$, $p<.01)$. These findings show that in terms of predictors identified Schaefer and Moos' model of PTG (1992), the most important predictor of PTG in this study was active coping which uniquely explained $21 \%$ of the variance in PTG.

Table 3.12 Summary of hierarchical regression for Schaefer and Moos' (1992) model of PTG

\begin{tabular}{|c|c|c|c|c|c|}
\hline & Adjusted $\mathrm{R}^{2}$ & $F$ & B & SE B & $\beta$ \\
\hline $\begin{array}{l}\text { Model } 1 \\
\text { Active Coping T1 }\end{array}$ & 0.21 & $12.26^{*}$ & 3.28 & 0.94 & $0.48^{*}$ \\
\hline $\begin{array}{l}\text { Model } 2 \\
\text { Active Coping T1 } \\
\text { Age }\end{array}$ & 0.34 & 11.99* & $\begin{array}{l}3.35 \\
-1.00\end{array}$ & $\begin{array}{l}0.86 \\
0.33\end{array}$ & $\begin{array}{l}0.49 * \\
-0.38 *\end{array}$ \\
\hline $\begin{array}{l}\text { Model } 3 \\
\text { Active Coping T1 } \\
\text { Age } \\
\text { Social Support T1 }\end{array}$ & 0.34 & $8.32 *$ & $\begin{array}{l}3.26 \\
-0.94 \\
0.40\end{array}$ & $\begin{array}{l}0.86 \\
0.34 \\
0.41\end{array}$ & $\begin{array}{l}0.48^{*} \\
-0.36^{*} \\
0.13\end{array}$ \\
\hline $\begin{array}{l}\text { Model } 4 \\
\text { Active Coping T1 } \\
\text { Age } \\
\text { Social Support T1 } \\
\text { Daily functioning T1 }\end{array}$ & 0.33 & $6.23 *$ & $\begin{array}{l}3.26 \\
-0.90 \\
0.41 \\
0.66\end{array}$ & $\begin{array}{l}0.87 \\
0.35 \\
0.41 \\
1.07\end{array}$ & $\begin{array}{l}0.48^{*} \\
-0.34^{*} \\
0.13 \\
0.08\end{array}$ \\
\hline
\end{tabular}

$* p<.05 ;$ Std Error $B=$ standard error of $B ; B=$ standardized beta.

\subsubsection{Exploratory model and this sample}

A stepwise linear regression was conducted to generate a model of PTG which accounted for the variables at time 1 which were most predictive of PTG at time 2. This method also allowed for PTSS and denial coping to be considered within a model of PTG as these variables are not included in models of PTG in the literature (Tedeschi \& Calhoun, 2004; Schaefer \& Moos, 1998). As Table 3.13 shows, active coping explained $21 \%$ of the variance in PTG $(F(1,42)=$ $12.26, p<.001)$, age accounted for $13 \%$ of the variance in PTG $(F(2,42)=11.99, p<.001)$ and rumination accounted for $14 \%$ of the variance in PTG $(F(5,42)=15.22, P<.001)$. None of the other predictors significantly contribute to explaining the variance in this model. 
Table 3.13 Summary of stepwise regression findings using all variables as predictors of PTGI at time 2 .

\begin{tabular}{|l|l|l|l|l|l|}
\hline & Adjusted R & F & B & SE B & $\beta$ \\
\hline $\begin{array}{l}\text { Model 1 } \\
\text { Active Coping T1 }\end{array}$ & 0.21 & $12.26^{*}$ & 3.28 & 0.94 & $0.48^{*}$ \\
\hline $\begin{array}{l}\text { Model 2 } \\
\text { Active Coping T1 } \\
\text { Age }\end{array}$ & 0.34 & $11.99^{*}$ & 3.35 & 0.86 & $0.49^{*}$ \\
\hline $\begin{array}{l}\text { Model 3 } \\
\text { Active Coping T1 }\end{array}$ & & & -1.00 & 0.33 & $-0.38^{*}$ \\
$\begin{array}{l}\text { Age } \\
\text { Social Support }\end{array}$ & 0.34 & $8.32^{*}$ & 3.26 & 0.86 & $0.48^{*}$ \\
\hline $\begin{array}{l}\text { Model 4 } \\
\text { Active Coping T1 }\end{array}$ & & & -0.94 & 0.34 & $-0.36^{*}$ \\
Age & 0.34 & 11.99 & 0.40 & 0.41 & 0.13 \\
\hline $\begin{array}{l}\text { Model 5 } \\
\text { Active Coping T1 } \\
\text { Age } \\
\text { Rumination T1 }\end{array}$ & & & $\begin{array}{l}3.35 \\
-1.00\end{array}$ & $\begin{array}{l}0.86 \\
0.33\end{array}$ & $\begin{array}{l}0.49^{*} \\
-0.38^{*}\end{array}$ \\
\hline
\end{tabular}

$* p<.05 ;$ Std Error $B=$ standard error of $B ; \beta=$ standardized beta.

\subsubsection{Summary of findings from regression analyses}

Both models (Tedeschi \& Calhoun, 2004; Schaefer \& Moos, 1992) predicted limited variance in PTGI at time 2. The predictors identified by Schaefer and Moos (1998) explained 34\% of the variance in PTG at time 2. In contrast, the predictors identified by Tedeschi and Calhoun $(1995,2004)$ explained $43 \%$ of the variance in PTG at time 2 . However, Rumination at time 1 was the only factor from Tedeschi and Calhoun's model (2004) which predicted variance in PTGI at time 2 (i.e. 37\% variance). A stepwise regression revealed that denial coping and PTSS did not appear to account for variance in PTGI at time 2. An exploratory model of PTG was developed which accounted for $48 \%$ of the variance in PTGI at time 2 and the predictors at time 1 which best explained PTG at time 2 were active coping, age and rumination. 


\title{
Chapter Four
}

\author{
Discussion
}

\subsection{Overview}

In this chapter, the main findings of this study will be discussed with reference to the key research questions and hypotheses underpinning this thesis. This study had several key research questions. Firstly, to determine whether posttraumatic growth (PTG) occurred in a stroke survivor sample over time and to compare the level of growth reported with findings published in similar studies. Secondly, to use correlational analyses to examine the relationship between PTG and a range of predictor variables over time which include social support, active coping, acceptance coping, denial coping, daily functioning, posttraumatic stress symptoms (PTSS) and rumination. Thirdly, to use mediation analyses to determine whether active coping and rumination were mediators of the relationship between social support and PTG. Fourthly, to determine whether rumination mediates the effect of PTSS on PTG. Finally, to use regression analyses to determine the predictive utility of known models of PTG (Tedeschi \& Calhoun, 2004; Schaefer \& Moos, 1998) and to evaluate which variables at time 1 were most predictive of PTG at time 2.

This chapter contains the following: a summary of the main study findings, an appraisal of the study findings in light of the relevant literature, an evaluation of the theoretical implications, a critical review of the strengths and limitations of the study, as well as an evaluation of the clinical implications of the findings and recommendations for future research.

\subsection{Summary of the main study findings}

This study is the first known study to demonstrate the development of PTG over time in a stroke survivor sample in the UK. Using the PTGI to measure PTG, this study found evidence of PTG at two different time points: time 1 was approximately five months post-stroke, 
whereas time 2 was approximately eleven months post-stroke. As predicted, participants experienced significantly more growth at time 2 compared to time 1. PTGI subscale analysis revealed that participants experienced significantly more growth at time 2 compared to time 1 across all the domains of PTG: new possibilities, relating to others, personal strength, spiritual change and appreciation of life. These findings are suggestive of a developmental trajectory toward PTG as stroke survivors experienced significantly more PTG, across all domains, at time 2 compared to time 1 . These findings also contribute to the literature on PTG more generally.

In terms of the main findings regarding the role of relationships and PTG, correlational analyses revealed that social support was positively associated with the PTG subscale 'relating to others' at time 1. However, social support at time 1 was not associated with PTG at time 2. As predicted, social support at time 2 was positively associated with PTG at time 2 . This pattern of findings suggests that stroke survivors who felt social supported, at time 1, were more likely to perceive improved relating to others in the early acute period post-stroke. At time 2 , stroke survivors who felt socially supported reported a more global experience of PTG. In addition, stroke survivors who were more functionally independent at time 2 were more likely to perceive 'new possibilities' at time 2.

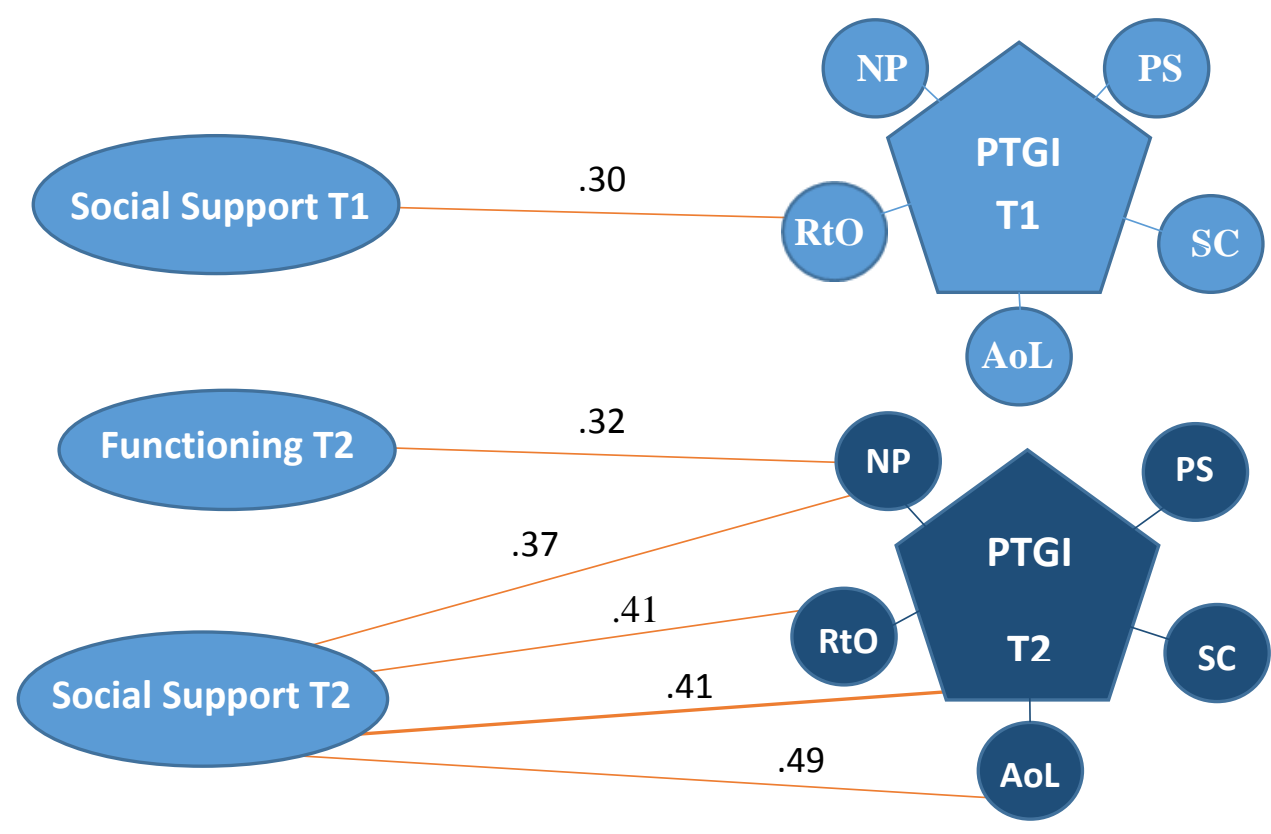

Figure 4.1 Path illustration of key correlational findings pertaining to social support, functioning and PTG.

(NP = New Possibilities; RtO = Relating to Others; PS = Personal Strength; AoL = Appreciation of Life; $\mathbf{S C}=$ Spiritual Change $)$ 
In terms of psychological adjustment factors and PTG, this study found that active coping at time 1 was positively associated with PTG at time 2. Active coping was also associated with PTG at time 1 and time 2. Neither acceptance coping nor denial coping were significantly associated with PTG. Interestingly, denial coping at time 1 was associated with increased 'appreciation of life' and rumination at time 2. This pattern of findings shows that stroke survivors who took steps toward overcoming their problems at time 1 were more likely to experience PTG at time 2. This study also suggests that the more stroke survivors engaged in a process of denial coping at time 1 , the more likely they were to ruminate and experience an increased appreciation of life at time 2 . Of note, mediation analysis revealed that active coping did not mediate the relationship between social support and PTG.

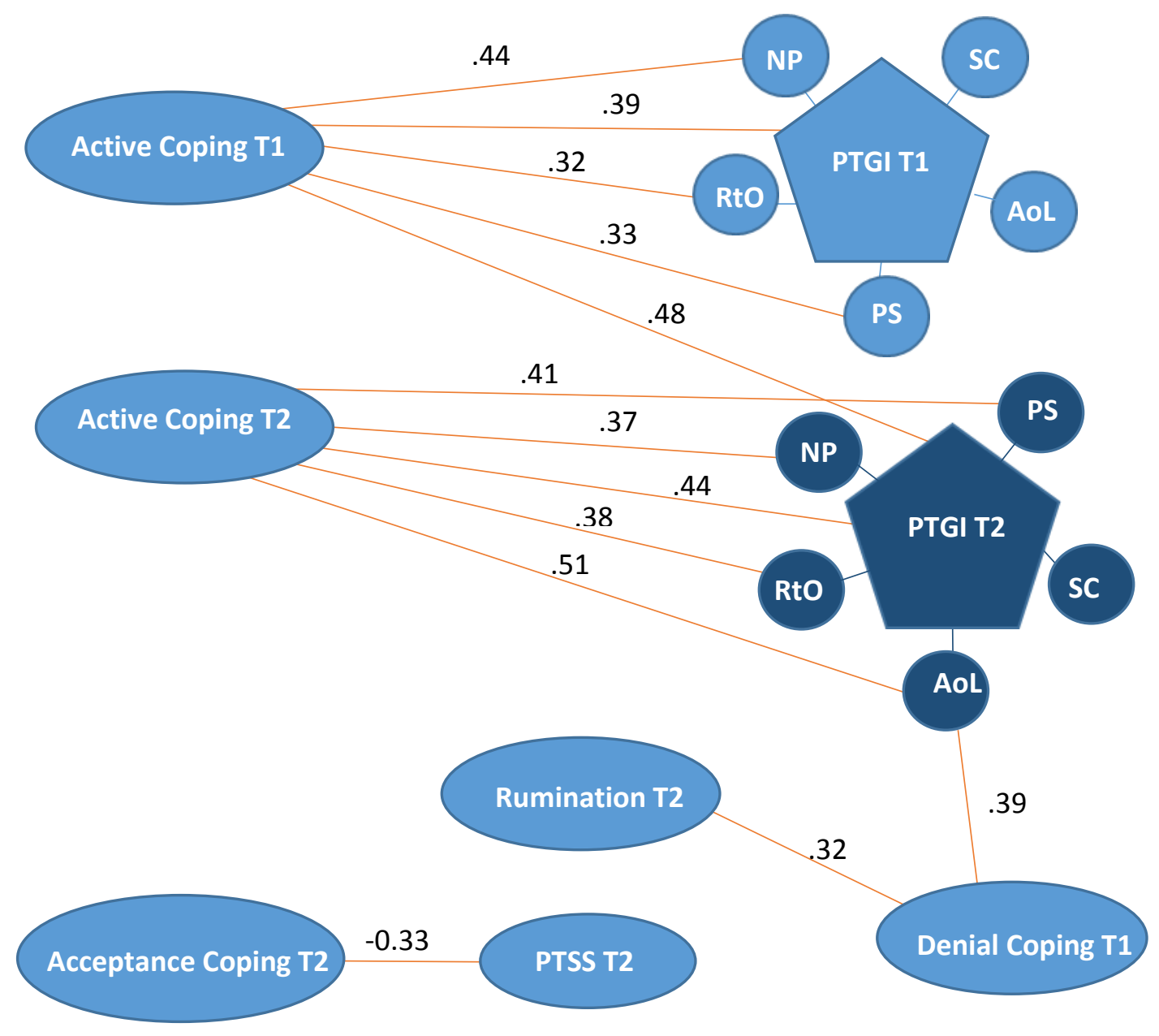

Figure 4.2 Path illustration of key correlational findings pertaining to psychological adjustment and PTG.

( NP = New Possibilities; RtO = Relating to Others; PS = Personal Strength; $\mathbf{A o L}=$ Appreciation of Life; $\mathbf{S C}=$ Spiritual Change $)$ 
In terms of cognitive processing and PTG, this study found that consistent with our prediction, rumination was associated with PTG at time 1 and time 2. Stroke survivors who experienced rumination at time 1 were also more likely to experience PTG at time 2 . Interestingly, this study found that rumination was associated with PTSS at time 1 and time 2. This pattern of findings provides evidence in support of the 'struggle with trauma' theorised by Tedeschi and Calhoun (2004) as key to facilitating PTG.

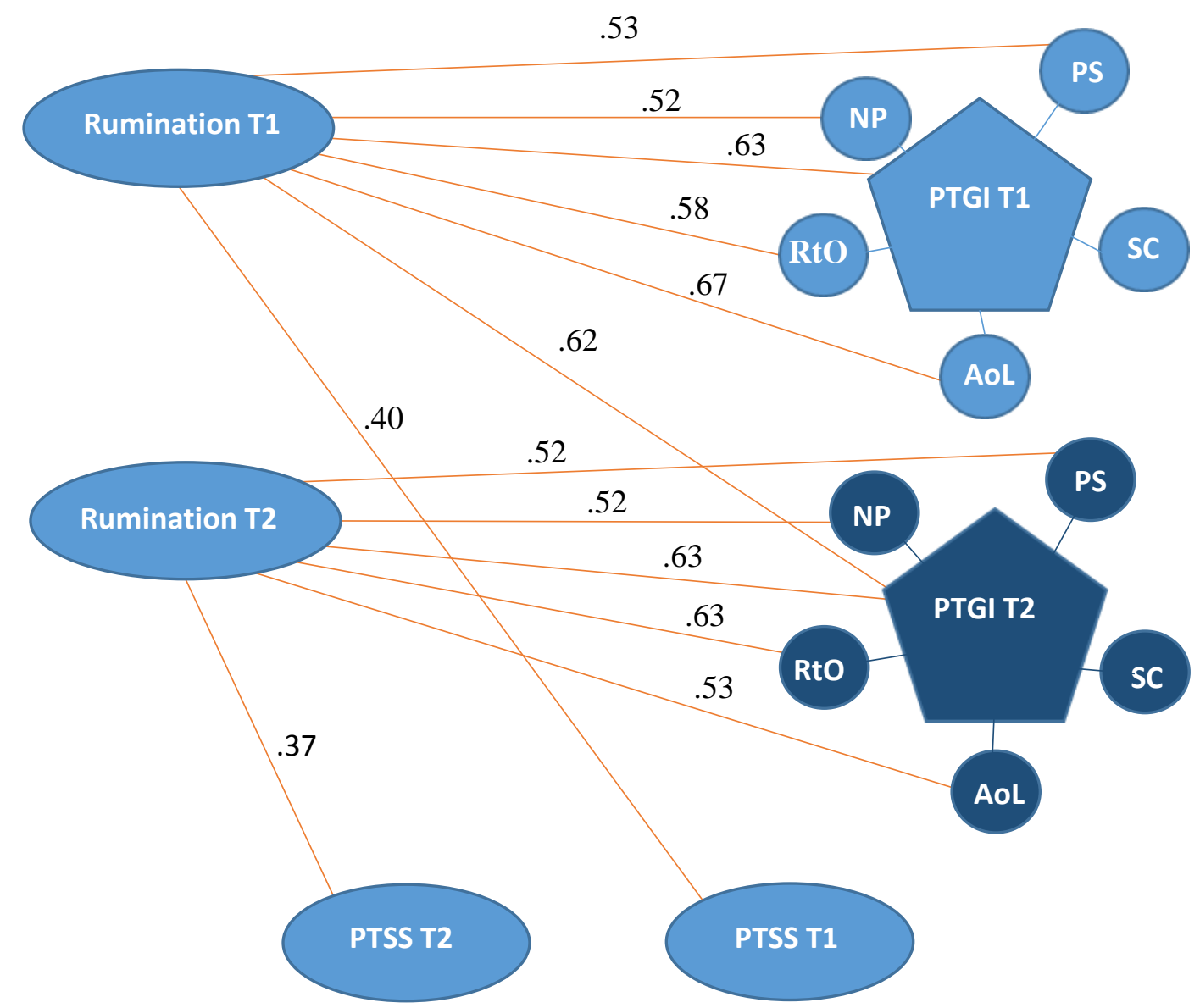

Figure 4.3 Path illustration of key correlational findings pertaining to cognitive processing factors and PTG.

(NP = New Possibilities; RtO = Relating to Others; PS = Personal Strength; AoL $=$ Appreciation of Life; $\mathbf{S C}=$ Spiritual Change $)$

Mediation analysis revealed that rumination mediated the effect of PTSS on PTG over time which broadly supports the model of PTG proposed by Tedeschi and Calhoun $(1995,2004)$. Notably, this study found that rumination did not mediate the effect of social support on PTG. Finally, regression analyses revealed that neither model of PTG (Tedeschi \& Calhoun, 2004; Schaefer \& Moos, 1998) fit neatly with the data in this study. Exploratory regression analyses revealed that rumination, active coping and age were most predictive of PTGI at time 2 . 


\subsection{Main study findings and their relation to past research}

\subsubsection{PTG in stroke survivors over time}

This study is the first to demonstrate the development of PTG over time using a stroke survivor sample in the UK. Stroke survivors were shown to have developed significantly more PTG at follow-up (i.e. six months after time 1). No comparable studies in the literature have focused on the development of PTG longitudinally in stroke survivors. However, studies examining the development of PTG in other traumata have had mixed findings (Cordova et al. 2001; Park et al. 1996). Following a review of the correlational and longitudinal evidence, Linley and Joseph (2004) concluded that when the severity of a traumatic event is experienced as significant, PTG is more likely to occur, provided that an individual draws on personal resources and cognitively processes the trauma. In this way, it is unclear whether PTG develops as a result of intervening events and processes (e.g. cognitive processing), the severity of the traumatic event, the temporal relationship between the traumatic event and PTG or some combination therein. This is compounded by the finding that age was inversely associated with PTG at time 2. Nevertheless, the findings in the current study contribute to a growing body of literature, albeit mainly cross-sectional in design, which shows that PTG can occur in a stroke survivor population (Gangstad et al. 2009; Kuenemund et al. 2014).

Although the mean PTGI score in this study did not differ significantly from the mean PTGI scores cited in similar stroke research, the qualitative nature of these comparisons are noteworthy. The mean PTGI score reported in this study is similar to what Gangstad et al. (2009) and Kuenemund et al. (2014) found but the mean age reported in the latter is much younger than the sample in this study. Studies investigating PTG in stroke survivors (Weiss, 2004) and MI survivors (Bluvstein et al. 2012) report similar levels of growth, but again the mean age of participants is considerably younger than the sample in this study. Nevertheless, this study shows that PTG can occur in a sample of stroke survivors where the mean age is approximately 75 years old. This finding, coupled with the unexpected occurrence of PTG at time 1 (i.e. approximately five months post-stroke) has implications for how the developmental trajectory of PTG is understood post-stroke and how PTG is conceptualised more generally in stroke survivors. 
The age range of stroke survivors in this study may have influenced the developmental trajectory of PTG and the level of growth reported at time 1. Tedeschi and Calhoun (2004) theorise that the development of PTG involves an iterative process which can include gradual and abrupt shifts toward growth. They recognise that there are no uniform trajectories toward growth and suggest that their model merely provides a framework for explaining PTG (Calhoun \& Tedeschi, 2004). Elsewhere in the literature, Aldwin and Levenson (2004) have described the process of PTG in older people as somewhat akin to the development of wisdom whereby the loss associated with the traumatic event not only triggers PTG but also enables a person to let go of existing unhelpful assumptions about the world. Tornstam (1994) has proposed that a traumatic event or crisis can serve as a precursor to gerotranscendence. The term gerotranscendence is used to describe the process whereby an older person shifts from a materialistic or rational perspective reliant on external factors, to a transcendental, reflective perspective on life (Tornstam, 1994). While these findings appear to fit conceptually with PTG and may account for the developmental nuances of an older sample, it is unclear whether the aforementioned reappraisal processes lead to quantifiable PTG (Calhoun \& Tedeschi, 2004).

As predicted, this study found that significantly more PTG was reported at time 2 (i.e. approximately eleven months post-stroke). Interestingly, this study also found that a considerable amount of PTG had occurred by time 1 (i.e. approximately five months poststroke). This finding is consistent with some of the literature which suggests that reported levels of growth tend to remain consistent between two and twelve months after a traumatic event (Frazier et al. 2001; Linley \& Joseph, 2004). In the period between time 1 and time 2, stroke survivors may have experienced changes in daily functioning, health, as well as changes in their relationships and social role. According to the literature, such interim changes are likely to encourage stroke survivors to engage in cognitive and emotional processing of the traumatic event which can lead to PTG (Tedeschi \& Calhoun, 2004). Therefore, it is unlikely that any such interim changes would have resulted in a 'pile-up' of major losses that interfere with the potential for PTG (Harvey et al. 2004).

Analyses of the PTGI subscales revealed that all of the subscales were significantly higher at time 2, compared to time 1. Janoff-Bulman (2004) suggests that whilst the five subscales of PTGI appear to reflect the lived experience of positive change in the aftermath of trauma, 
these subscales are not necessarily underpinned by the same model of PTG. However, Tedeschi and Calhoun (2004) argue that the different aspects of PTG, as captured in the PTGI subscales, are related to cognitive and emotional processing in different ways. For example, changes in 'relating to others' may reflect the increased likelihood that an individual feels able to disclose intrusive thoughts and feelings to others as a way of regulating these feelings. Of note, Tedeschi and Calhoun (1996) have found that the PTGI subscales of 'new possibilities' and 'personal strength' are associated with openness to experience. It has been suggested that changes in the 'personal strength' subscale do not reflect schema change. However, in this study, there was a significant change in 'personal strength' between time 1 and 2 .

\subsubsection{Daily functioning and PTG}

This study found that daily functioning was not significantly associated with PTG at time 1 or 2. Nor was daily functioning at time 1 associated with PTG at time 2. However, daily functioning at time 2 was positively associated with the PTGI subscale 'new possibilities'. This suggests that higher levels of daily functioning are associated with the perception of new possibilities in life at approximately eleven months post-stroke. However, this finding tells us little about the role of daily functioning in the acute period post-stroke. Interestingly, this study found that daily functioning remained relatively stable between time 1 and time 2 and there was a trend toward independent functioning for most participants. This suggests that this sample of stroke survivors did not experience physical disability to such an extent that it impacted on their ability to perform most activities of daily living. Collectively, these findings show that stroke survivors' daily functioning remained relatively stable and was not associated with PTG apart from the domain of 'new possibilities' which were perceived as emerging over time.

There is a dearth of literature examining the relationship between daily functioning and PTG and therefore, a lack of comparable findings. However, the relationship between these variables is worthy of further consideration given the physical disability which commonly follows a stroke and its impact on daily functioning. According to Schaefer and Moos (1998), difficulties in daily functioning are negatively associated with PTG. However, Tedeschi and Calhoun (1999) propose that trauma survivors who experience long-lasting consequences are 
likely to experience more stress which can lead to significant psychological distress and through processing this can develop PTG. They also posit that the developmental stage an individual is at when a traumatic event occurs impacts on how they process their experience. It is possible that this is of particular relevance in the case of samples which contain older people who may be experiencing a trauma in the context of other developmental tasks (e.g. adjusting to multiple losses, social change). Interestingly, some studies have found that survivors of trauma are at increased risk of experiencing physical illness in the acute period following trauma (Herbert \& Cohen, 1993). This literature suggests that irrespective of physical disability, stroke survivors may be at increased risk of experiencing physical illness post-stroke. Although the findings in this study do not provide unitary support for either model of PTG, further research is needed in order to further elucidate the interface between mind and body in relation to the development of PTG.

\subsubsection{Social support and PTG}

As predicted, social support at time 2 was positively associated with PTG at time 2 . This shows that stroke survivors who experienced PTG at around eleven months post-stroke were more likely to feel socially supported. Interestingly, the perception of social support, at time 1 , was only positively associated with the PTGI subscale 'relating to others'. This suggests that stroke survivors who felt socially supported at around five months post-stroke were more likely to experience an improvement in their experience of social relationships, but did not experience other aspects of PTG. No significant association was found between the perception of social support at time 1 and PTG at time 2, apart from the PTGI subscales of 'personal strength' and 'appreciation of life'. These findings suggest that participants who felt socially supported at around five months post-stroke were likely to experience increased personal strength and appreciation of life eleven months post-stroke. Collectively, these findings suggest that although social support is associated with PTG at around eleven months post-stroke, the relationship between social support and PTG at five months post-stroke is less clear. Nevertheless, these findings point toward the role of social support and reappraisal of social relationships in the acute post-stroke phase. 
There are no known comparable studies in the stroke literature which measure the relationship between social support and PTG. However, studies in comparable literature have had mixed findings regarding the relationship between social support and PTG across a range of traumata (Stanton et al. 2005; Prati et al. 2009; Helgeson et al. 2006; Park et al. 2010). The literature suggests that social support leads to positive rehabilitation outcomes in $\mathrm{MI}$ survivors and improved psychological adjustment in cancer survivors (Moos, 1985). However, data were not collected on whether participants were receiving any rehabilitation or accessing any peer support interventions in this study. Although there is a lack of research investigating the role of social support in PTG, social support forms part of common models of PTG (Tedeschi \& Calhoun, 2004; Schaefer \& Moos, 1998). According to the model of PTG proposed by Tedeschi and Calhoun $(1995,2004)$, social support has an indirect effect on PTG whereby trauma survivors who feel supported by others are more likely to ruminate over their experiences and report PTG. Therefore, although data were not collected on objective measures of social support, access to social support, support-seeking attributes or satisfaction with support received, these variables are likely to be negligible given that PTG is proposed to be dependent on perceived social support. Another reason for measuring perceived social support is that social support may not always be helpful. For example, studies have found that when those in a trauma survivors social network respond to that survivor's emotional distress by trying to block the expression of such feelings, giving advice or attempting to identify with those feelings, this can lead to a trauma survivor feeling upset and unsupported (Ingram et al. 2001; Lehman et al. 1986; Wortman, 2004)

According to the model of PTG proposed by Schaefer and Moos (1992), social support promotes PTG through facilitating the development of effective coping strategies. Perception of social support was not significantly associated with active coping, acceptance coping or denial coping. Nor were rumination or daily functioning significantly associated with social support. This pattern of findings suggests that social support was not directly associated with rumination or types of coping which is somewhat inconsistent with the models of PTG postulated by Tedeschi and Calhoun (2004) and Schaefer and Moos (1992). However, the role of these factors in indirectly influencing PTG is discussed in section 4.3.7. 


\subsubsection{Coping and PTG}

The current study did not identify any significant relationship between acceptance coping or denial coping and PTG. However, active coping was significantly associated with PTG at time 1 and time 2 . Of note, active coping at time 1 was also associated with PTGI at time 2 but was only significantly associated with the PTGI subscales of 'new possibilities', 'relating to others' and 'personal strength'. At time 2, active coping was significantly associated with the PTGI subscales of 'new possibilities', 'relating to others', 'personal strength' and 'appreciation of life'. This pattern of findings suggests that active coping at approximately five months post stroke was associated with the perception of new possibilities, personal strength and improved relationships at eleven months post-stroke. However, between five and eleven months post-stroke, active coping also became associated with increased 'appreciation of life'.

These findings are consistent with comparable studies of different traumata which report significant associations between active coping and PTG (Morris et al. 2007), but inconsistent with studies which have found a positive association between PTG and acceptance coping (Park et al. 1996). Of note, studies in the stroke literature have found a positive association between denial, as measured by the Cognitive Processing of Trauma Scale (Williams et al. 2002), and PTG in stroke survivors (Gangstad et al. 2009). Using a study of stroke carers, Hallam and Morris (2014) also found a positive association between denial coping, as measured by the COPE scale (Carver et al. 1989), and PTG (Hallam \& Morris, 2014).

Schaefer and Moos (1998) propose that active rather than denial coping is involved in the development of PTG, whereas Tedeschi and Calhoun (2004) propose that rumination, or meaning-focused coping, facilitates the development of PTG. Tedeschi and Calhoun (2004) also suggest that acceptance coping in the acute phase may be beneficial. There are mixed findings in the literature regarding the relationship between denial coping and PTG (see Zoellner \& Maercker, 2006). According to Litman (2006), active coping is typified by taking steps to eliminate the problem, acceptance coping involves learning to accept the problem, whereas denial coping is typified by refusing to believe the problem is real. It has been suggested that the active pursuit of growth may serve as an intentional coping strategy in and of itself (Nolan-Hoeksema \& Davis, 2004). In this way, reported PTG may reflect cognitive 
illusions which serve to protect or enhance an individual's effort at active coping as a way of improving mood (Nolen-Hoeksema \& Davis, 2004). According to the literature, rumination type coping can be associated with negative outcomes (Nolen-Hoeksema \& Larson, 1999). However, it has been suggested that there may be different types of rumination coping where reflective type rumination is associated with positive outcomes and brooding type rumination is associated with poorer outcomes (Nolen-Hoeksema \& Davis, 2004; Treynor et al. 2003). However, it is unclear whether reflection can develop into brooding type coping and vice versa.

\subsubsection{Rumination and PTG}

As predicted, rumination was positively associated with PTG at time 1 and time 2. Of note, rumination at time 1 was significantly associated with PTGI at time 2 and significantly correlated with all PTGI subscales apart from 'spiritual change'. However, no significant association was found between rumination and acceptance coping. Interestingly, denial coping at five months was positively associated with rumination at eleven months. Collectively, these findings show that rumination at approximately five months and eleven months post-stroke was associated with the development of PTG over time. Interestingly, denial coping at five months was associated with rumination at eleven months.

These findings lend support to previous studies which have also found that rumination is associated with PTG (Taku et al. 2009; Calhoun et al. 2000). According to Tedeschi and Calhoun (2004), rumination can be either automatic or deliberate and can occur soon after the traumatic event, or on a more recent basis. They theorise that emotional distress following a traumatic event initiates an iterative process of rumination and attempts to engage in coping behaviour in order to reduce psychological distress. They propose that rumination is more automatic than deliberate in the acute phase post-trauma but then becomes more deliberate following the experience of coping successes and the reduction of psychological distress. In this way, rumination has the potential to be constructive or destructive and can impact on outcomes. Tedeschi and Calhoun (2004) posit that the process of cognitive processing or rumination is effortful and takes time. The findings in this study suggest that rumination was persistent and sustained over a six month period. However, this 
study also found that stroke survivors reported a degree of PTG at five months post-stroke. Contrary to the model proposed by Tedeschi and Calhoun (2004), some studies have found that PTG may occur soon after a traumatic event but the growth may decline over time. For example, using a mixed design Frazier et al. (2001) found that survivors of sexual assault reported PTG two weeks after the event but no longer reported PTG in interviews several weeks later. This highlights that there may be a potential cost to cognitive processing or rumination in the acute phase following a traumatic event (Wortman, 2004). Nevertheless, the findings in this study suggest a developmental trajectory consistent with the model proposed by Tedeschi and Calhoun (2004) whereby PTG increased over time.

Studies have demonstrated that recent, deliberate rumination best predicts PTG in survivors of a range of different traumata (Taku et al. 2009). However, it is unclear whether it is the process or outcome of rumination that facilitates the development of PTG and this is critical in terms of conceptualising PTG (Zoellner \& Maercker, 2004). One of the limitations of the rumination scale used was that it did not produce subscale scores for automatic and deliberate rumination. While there are measures available which capture both aspects of rumination (e.g. Event related rumination inventory; Cann et al. 2011), it is unclear how valid such measures are given that it is likely respondents may be less aware of automatic rumination which would impact on their recall of rumination.

Rather unexpectedly, this study found that denial coping at approximately five months poststroke was positively associated with rumination at eleven months post-stroke. This suggests that attempts to reject the reality of stroke at five months were associated with increased rumination at eleven months post-stroke. These findings are inconsistent with both models of PTG (Tedeschi \& Calhoun, 2004; Schaefer \& Moos, 1998) which propose that denial coping is negatively associated with PTG. Although some studies found that denial coping can be associated with PTG (Gangstad et al. 1999; Hallam \& Morris, 2014), there is a paucity of literature regarding the relationship between denial coping and rumination in the context of posttraumatic growth. The dual process model (Stroebe \& Schut, 1999) provides a framework for explaining the grieving process following a trauma experience. According to this model, adaptive coping is achieved through alternating between confronting and avoiding thoughts about the loss. In this way, it is possible that denial coping provides trauma survivors a brief 
respite from stress. This model accounts for why stroke survivors might engage in rumination type coping on some occasions, and more avoidance-based, denial coping on other occasions.

\subsubsection{PTSS and PTG}

This study found that contrary to our hypothesis, PTSS was not significantly correlated with PTG at time 1 or time 2. However, PTSS was associated with rumination at time 1 and time 2. In terms of the two rumination subscales (i.e. rumination soon after the event and recent rumination), this study also found that PTSS was associated with recent rumination at time 2 . This pattern of findings suggests that the more stroke survivors experienced psychological distress at eleven months post-stroke, the more they engaged in ruminating about their experience. Interestingly, stroke survivors at eleven months post-stroke were more likely to engage in recent rumination which suggests that it may have been more deliberate in nature. Of note, this study also found that stroke survivors were more likely to engage in acceptance coping at eleven months post-stroke if they were experiencing less psychological distress regarding the trauma. This finding is consistent with the model of PTG proposed by Tedeschi and Calhoun (2004) where acceptance coping is associated with the outcome PTG and viewed as an outcome of 'a struggle with trauma'.

According to the literature, some studies have found a positive association between PTSS and PTG (Morrill et al. 2008; Park et al. 1996), whereas others have found a negative association (Frazier et al. 2001). In a longitudinal study of trauma survivors, McMillen et al. (1997) found that reports of PTG 4-6 weeks following a trauma were predictive of fewer PTSS three years later. Interestingly, they also found that survivors who reported PTG had less psychiatric diagnoses despite increased trauma severity, whereas survivors who did not report growth had increased psychiatric diagnoses as the trauma severity increased. These findings suggest that there may have been a buffering effect of perceived PTG when the traumatic event was perceived as severely traumatic. Given the mixed findings regarding the relationship between PTSS and PTG, further research is required using different ways of measuring PTSS in the posttrauma period. 
One of the striking findings of this study was that rather than PTSS being associated with PTG, it was associated with rumination at approximately five months and eleven months poststroke. However, there is little to draw on in terms of comparable findings in the PTG literature. According to the posttraumatic stress disorder literature (PTSD), studies have found that trauma related rumination serves as a dysfunctional coping strategy which leads to poor outcomes (Ehring \& Ehlers, 2014; Ehlers \& Clark, 2007; Wells \& Sembi, 2004). However, from an ideological standpoint, the positive association between rumination and the experience of trauma-related distress appears to fit with the description Tedeschi and Calhoun (2004) give of this process involving 'a struggle with trauma' which is necessary for PTG to occur. They propose that the emotional regulation difficulties which follow a traumatic event can be characterised as a 'struggle' which can continue to co-exist, to a greater or lesser degree, alongside PTG. In this way, our finding that PTSS and rumination sustained over time also fits with the model outlined by Tedeschi and Calhoun (2004) and suggests that supports our finding that PTSS had an indirect effect on PTG (see section 4.3.9). The findings in this study highlight that further research is required to learn more about the role of PTSS and the development of PTG post-trauma.

The findings in this study also raise some wider theoretical questions, particularly regarding the role of resilience and positive emotions in PTG. Although Tedeschi and Calhoun (2004) argue that individuals who are resilient may be less likely to experience PTG, it is also possible that resilient individuals may be able to cope with a higher threshold of stress without experiencing distress and thus make them more likely to experience PTG (Aldwin \& Levenson, 2004). According to Tedeschi and Calhoun (2004), resilience differs from PTG in that the latter constitutes a qualitative change in functioning. In this way, it is unclear whether resilient individuals experience growth following trauma as a qualitative change in functioning.

Although the construct of PTG has stemmed from the movement of positive psychology, little is known about the role of positive emotions in PTG (Seligman \& Csikszentmihaly, 2000). The literature suggests that positive mood is predictive of positive health outcomes (e.g. risk of stroke; Ostir et al. 2001). Frederickson (2001) proposes that the means through which positive emotions lead to positive outcomes involves processes such as the experience of less distress, cognitive re-evaluation of priorities and values, as well as drawing upon coping processes and personal resources. Frederickson et al. (2003) found that positive emotions following the 
traumatic experience of a terror attack mediated the relationship between pre-existing resilience and psychological growth, as well as symptoms associated with depression. These findings from the positive psychology literature suggest that there may be a reciprocal relationship between positive emotions and PTG which requires further study.

\subsubsection{Social support as a mediating variable}

Mediation analysis revealed that the relationship between social support and PTG was not mediated by active coping or acceptance coping at five months or eleven months post-stroke. Nor were the relationships between coping (i.e. active and acceptance) and PTG mediated by social support at five or eleven months post-stroke.

This study found that rather than indirectly affecting the relationship between coping and PTG, social support was directly associated with PTG at eleven months post stroke. This pattern of findings is consistent with the literature which suggests that social support directly affects the development of PTG. Stanton et al. (2006) have found that active coping and seeking social support in the acute phase post-trauma are unique predictors of PTG. This suggests that perhaps a clear sense of one's physical, social and emotional needs post-trauma is required in order to seek social support and engage in active coping and thus, facilitate PTG. Similarly, in a longitudinal study of cancer survivors, Scrignaro et al. (2011) have found that active coping and autonomy-supportive social support from care-givers is predictive of PTG over a six month time period. Interestingly, a study of PTG in stroke carers found that social support, but not active coping, is associated with PTG (Hallam \& Morris, 2014). In this way, the findings in this study do not entirely support Schaefer and Moos' (1998) theory that social support promotes PTG through the development of effective coping strategies. Although the findings in this study support the literature, it is unclear whether the indirect effect of social support, as theorised by Schaefer and Moos (1998), has been previously investigated in the context of PTG. It is possible that this pattern of findings fits with the model of PTG proposed by Tedeschi and Calhoun (2004) whereby social support facilitates the cognitive processing of a trauma. 


\subsubsection{Rumination as a mediating variable}

Mediation analysis revealed that the relationship between PTSS and PTG was mediated by rumination. More specifically, rumination at time 1 mediated the effect of PTSS at time 1 on PTG at time 1. Rumination at time 1 also mediated the effect of PTSS at time 1 on PTG at time 2. Rumination at time 2 also mediated the effect of PTSS at time 2 on PTG at time 2. This shows that rumination in the acute and later phases post-stroke has an indirect effect on the relationship between the experience of distress and the development of PTG. This finding lends support to the model of PTG proposed by Tedeschi and Calhoun (2004) which theorises that 'a struggle with trauma' involving both cognitive and emotional processing is required in order for PTG to develop. This suggests that PTSS in the acute and later phases post-stroke leads to the type of rumination necessary to facilitate PTG. It supports the theory that the intervening period between the traumatic event and the development of PTG may be characterised by 'a struggle with trauma' where stroke survivors may oscillate between attempts at coping, feelings of distress and experiences of growth (Tedeschi \& Calhoun, 2004). These mediational findings make a significant contribution to the literature on PTG. There are no comparable findings in the stroke survivor literature nor the PTG literature more generally. Studies of breast cancer survivors appear to have examined the role of PTSS independent of PTG (Shand et al. 2014). A cross-sectional study of breast cancer survivors found that positive cancer related rumination mediated the relationship between positive attentional bias and PTG (Chan et al. 2010). In contrast, the findings in this study demonstrate the role of rumination (i.e. positive and negative) in mediating the effect of PTSS (i.e. adaptive and maladaptive) on PTG longitudinally. Future research should aim to replicate these findings and extend these findings by examining whether rumination continues to mediate the effect of PTSS after a year post-trauma.

\subsubsection{Predictive model of PTG in this sample}

Hierarchical regression analyses revealed that the data produced models which were not entirely consistent with those proposed by Tedeschi and Calhoun (2004), as well as Schaefer and Moos (1998). This study found that rumination at time one was the only variable which significantly contributed to the model proposed by Tedeschi and Calhoun (2004) and 
accounted for $37 \%$ of the variance in total PTGI at time 2. This finding lends support to previous research which has also found that rumination is predictive of PTG (Hallam \& Morris, 2014). In contrast, only active coping and age significantly accounted for the variance in this study as per the model proposed by Schaefer and Moos (1998). A stepwise linear regression found that active coping, rumination and age were the best predictors of PTG in this study. Few studies in the literature have examined the predictors of PTG in light of the theoretical models of PTG proposed by Tedeschi and Calhoun (2004) and Schaefer and Moos (1998) and even fewer using a stroke survivor population, so there are therefore limited comparable findings. Further research is required to determine whether these models can adequately account for the development of PTG in stroke survivors over time.

\subsection{Summary of the theoretical implications of the current findings}

The results of this study suggest that the model of PTG proposed by Tedeschi and Calhoun $(1995,2004)$ offers a useful framework for conceptualising PTG in stroke survivors. This study contributes to a growing body of literature supporting the potential for PTG following health related traumatic events (e.g. Cordova et al. 2001). Stemming from the positive psychology movement (Seligman \& Csikszentmihalyi, 2000), the concept of PTG describes the transformative experiences of growth some individuals experience following trauma. Models of PTG (e.g. Schaefer \& Moos, 1992; Tedeschi \& Calhoun, 1995, 2004) provide a theoretical framework for understanding the mechanisms of transformative change inherent in PTG. The research questions examined in this thesis were derived from the theoretical predictions made by the models of PTG proposed by Schaefer and Moos (1992), as well as Tedeschi and Calhoun $(1995,2004)$.

At a meta-level, this study helps inform our understanding about the temporal relationship between traumatic events and PTG. Firstly, this study demonstrated the stability and extension of PTG in a sample of stroke survivors. This suggests that PTG can occur in the acute phase post stroke (i.e. up to five months) and extend over time (i.e. up to eleven months). Few studies have explored the development of PTG in the first year post-trauma, but there is emerging evidence in the literature to support these findings (Danhauer et al. 2013). Secondly, this study found evidence which supports the role of cognitive processing in PTG 
development and therefore, refutes the theory that PTG is a result of mere exposure of a trauma (Aldwin \& Levenson, 2004).

This study found predictors of PTG in stroke survivors but collectively these predictors do not appear to fit neatly with either model of PTG (i.e. Schaefer \& Moos, 1992; Tedeschi \& Calhoun, 1995, 2004): regression analyses revealed that active coping, rumination and age were most predictive of PTG. Nevertheless, one of the key findings in this study provides support for the model of PTG proposed by Tedeschi and Calhoun $(1995,2004)$. Mediation analyses revealed that rumination mediated the effect of PTSS on PTG over time. This is the first known study to demonstrate this relationship in the literature and further supports the theory that PTG involves effortful, deliberate cognitive processing over time. Critically, the findings in this study also lend support to Tedeschi and Calhoun's (2004) theory that the development of PTG involves a process of cognitive processing and emotional engagement which co-exist alongside attempts at coping and the residual distress of the traumatic event. Nonetheless, the role of individual factors such as optimism and the capacity for dialectical thinking in this process is less clear.

At a clinical level, the findings in this study appear to support the psychological challenges that an individual can face post-trauma, as conceptualised in the models of PTG (e.g. Tedeschi \& Calhoun, 1995, 2004). In clinical terms, stroke occurs suddenly and often leaves survivors with complex disability and rehabilitation needs (Adamson et al. 2004). In psychological terms, this can lead to significant changes in wellbeing, quality of life and relationships (Glass \& Maddox, 1992). In this way, the literature suggests that stroke can present a myriad of challenges both at a practical and psychological level. The results of this study appear to reflect the complex interplay of factors which are likely to impact on the development of PTG post-stroke. Interestingly, this study found that denial coping at time 1 was positively associated with rumination at time 2 . Other studies in the literature have found that denial coping is associated with PTG (Hallam \& Morris, 2014), but the association between denial coping and rumination over time suggests that denial may serve a protective function in the acute phase post-stroke. It is possible that in the acute phase post-trauma, efforts at active coping and cognitive processing of one's experience may co-exist alongside periods of denial coping. Further research is needed to clarify the functional role of denial coping post-trauma and the nature of its relationship to PTG longitudinally. 
The findings in this study provide limited support for the theoretical framework of PTG proposed by Schaefer and Moos (1992). Nevertheless, this study did find that active coping was a significant predictor of PTG. In the wider context of the main findings, it is possible that active coping operates parallel to the process of rumination proposed by Tedeschi and Calhoun $(1995,2004)$. In this way, engaging in active coping may provide opportunities for the coping successes that facilitate transformative change and as a result support the process of deliberate rumination necessary for PTG.

This study has emphasised individual experience of PTG and the perception of related variables and this tendency appears to be mirrored in the wider literature. Less is known about how systemic factors interact with factors such as cognitive processing in the development of PTG. While models of PTG acknowledge that PTG does not occur in a vacuum, it appears that the literature has placed less emphasis on the role of systemic variables such as social factors, relationships and culture. The impact of stroke extends from an individual to their most proximate and distal systems (e.g. family, community, social network) and a change in how an individual relates to such systems post-stroke may impact on the relative opportunities for coping successes and growth.

\subsection{Methodological strengths and limitations}

\subsubsection{Strengths}

A major strength of this study is that PTG was measured longitudinally using a sample of stroke survivors recruited through the National Health Service (NHS) in the UK. Previous research has examined PTG in stroke survivors in the UK using a cross-sectional design (Gangstad et al. 2009) and the only other known quantitative study examining PTG in stroke survivors was cross-sectional and used a German sample (Kuenemund et al. 2014). The PTG literature has been criticised for the paucity of research examining PTG longitudinally (Zoellner \& Maercker, 2006; Somerfield \& McCrae, 2000). Longitudinal studies examining the predictors of PTG over time have had mixed findings (Pollard \& Kennedy, 2007; Abraido-Lanza et al. 1998; Park et al. 1996) and this has highlighted the need for further research. This study bridges the gap between research on adaptational processes such as the stress and coping 
literature, and clinical psychology. The longitudinal design of this study involved measuring PTG and a range of related variables at two different points which enabled the predictors of PTG to be identified. Additionally, the difference between time 1 and time 2 was six months for all participants.

Another strength of this study is that participants were recruited through a local NHS stroke service which increased the likelihood that the sample was representative of the target population, as opposed to recruiting stroke survivors from a community setting (e.g. a Stroke Association group). As per the study protocol, the researcher supported participants in completing the questionnaires at time 1 and 2 . It is likely that this contributed to the lack of missing data, as well as the high response rate.

One of the key strengths of this study is that it allows for the testing of several theoretical predictions which underpin the models of PTG outlined by Tedeschi and Calhoun (2004) and Schaefer and Moos (1998). This study furthers our understanding regarding the development of PTG in the acute phase post-stroke and has identified predictors of PTG over time.

\subsubsection{Limitations}

\subsubsection{Population}

Although consecutive sampling was used to recruit stroke survivors from a clinical setting, it is unclear how representative the sample in this study is compared to the general population of stroke survivors. Given that the first point of contact with participants was via the clinical team, it is possible that some individuals who met the inclusion criteria were not invited to participate due to logistical factors or some may have been identified as unlikely to consider participation. The sample size recruited was also smaller than planned.

Several other factors may have impacted on the generalisability of the findings to other samples. Firstly, participants were required to have a certain degree of cognitive and language functioning in order to be able participate in the study. Although such eligibility criteria support the validity of the measures used, it is possible that they do not capture the sub population of stroke survivors who experience cognitive and language difficulties (Burton, 
2000). Secondly, although participants were asked whether they had accessed an intervention for mood related difficulties, this study did not measure the role of other areas of clinical intervention such as rehabilitation. Stroke survivors often experience complex disability post-stroke and may access rehabilitation post-stroke (Adamson et al. 2004). It is possible that stroke survivors' experience of rehabilitation may impact on the development of PTG. Thirdly, all participants identified themselves as 'White British' which makes it unclear whether these results are generalisable to populations which are more ethnically diverse.

\subsubsection{Methodological}

Although this study was longitudinal in design and accounted for the development of PTG in early phase post-stroke, there was not scope to study the development of PTG in the later phases post-stroke (e.g. 18-36 months post-stroke). Therefore, this study was unable to support the theoretical prediction that PTG often occurs in the period 18 to 36 months poststroke (Tedeschi \& Calhoun, 2004). Nevertheless, this study has contributed the literature on PTG in the acute phase post-stroke and provides preliminary support for the stability of PTG over time.

In terms of other design related limitations, this study did not use a control group nor did it account for possible intervening factors between time 1 and time 2 . Few studies have used control groups in the PTG literature and the efficacy of those studies which have employed such an approach (e.g. Kuenemund et al. 2014) is often limited due to the use of snowball sampling techniques to recruit control participants, as well as the use of a highly stressful event as a proxy for a traumatic event. Although the application of bootstrapping approaches across statistical tests is somewhat novel in the field of clinical psychology research, it appeared to provide a good fit with this study. It afforded this study greater statistical power which was beneficial due to the relatively low sample size (Wright et al. 2011). As the sample sizes in the systematic review demonstrate, it can be difficult to recruit stroke survivors and bootstrapping methods provide a way to reduce the risk of Type II error.

Given the findings of the priori power analysis, it is likely that the present study is somewhat underpowered due to the sample size. However, the literature suggests that modern robust statistical techniques such as bootstrapping confer benefits relative to parametric methods in terms of maintaining Type I error control and statistical power. In this way, despite the 
limitations of the sample size, power was optimised through using bootstrapping methods in hypothesis testing as this reduced the likelihood of Type I errors and also led to more accurate confidence intervals around robust effect size statistics (Erceg-Hurn \& Mirosevich, 2008). Rosenthal and Gaito (1963) report that statistically significant findings show that there has been sufficient power to detect an effect. This suggests that the significant findings in this study can be interpreted with reasonable accuracy. In addition, the reporting of confidence intervals shows that the effect sizes are wider and incorporate more error than if the power had been higher. The literature also suggests that feasible sample sizes can be reliably used to test multiple hypotheses (Lazzaronni \& Ray, 2012). Furthermore, it was decided not to use a statistical correction for multiple comparisons due to the inflated risk of Type II error (see section 2.6.3).

\subsubsection{Measures}

The reliance on self-report measures of the constructs in this study could be considered a limitation. According to Nisbett \& Wilson (1977), self-report measures can be inaccurate in measuring cognitive processes but are better able to measure constructs such as mood, attitudes and beliefs which require the ability to tap into short-term memory. Although selfreport measures may be prone to potential bias such as demand characteristics (Orne, 1962) and the halo effect (Thorndike, 1920), the validity of such measures hinge on the theoretical context in which hypotheses are derived and whether the measurement tool sufficiently measures the construct of interest (Haeffel \& Havard, 2010). Nonetheless, the finding that PTG was stable between times 1 and 2 and that it demonstrated a developmental trajectory discounts the likelihood of the halo effect occurring (Thorndike, 1920). In practical terms, selfreport measures are cost-effective, easy to administer and can be designed such that the measures used do not cognitively overload participants. In spite of the established construct validity for the PTGI (Tedeschi \& Calhoun, 1996), a degree of insight is required in order to engage with the items outlined in this measure. Nevertheless, in the case of the PTGI, it is the perception of experience that is the focus of measurement rather than the objective nature of experience. Briere and Elliot (2000) have found that subjective experience best predicts trauma following exposure to a traumatic event, rather than the nature of the traumatic event itself. 


\subsubsection{Confounding variables}

Efforts were made to control for the potential confounding effects of cognitive and language difficulties, as well as previous stroke. However, this study did not account for the relative timing of the traumatic event for an individual and this may have been a confounding factor. It is possible that having a stroke may have coincided with other major life events in participants' lives. The quantitative design of this study did not allow for such experiences to be captured in the data. Perhaps, timing of the traumatic event for an individual within the context of systemic and life-cycle factors could be best investigated using a qualitative design where the role of such factors in PTG provides the focus for investigation. The clinical research literature supports the use of qualitative research methods as a rigorous means of capturing the intricacies of human experience (Polkinghome, 2005). The sample of stroke survivors recruited for this study appeared to be functioning at a relatively high level of functional independence which does not appear to be entirely consistent with the range of disability that can be experienced post-stroke (Adamson et al. 2004).

Despite the apparent integrity of the construct validity of the PTGI (Tedeschi \& Calhoun, 1996) in this study, it is possible that other factors were not measured. Although different types of social support were measured at two time points post-stroke, it is possible that the social support needs of participants changed over time. Similarly, previous experience of trauma and the potential for pre-existing PTG were not accounted for in this study. According to the literature, people with previous experience may be less prone to PTG following new trauma because resilience may be enhanced in the acute post-trauma period and there may have already been a shift in core beliefs (Tedeschi \& Calhoun, 2004).

\subsection{Clinical and Service implications}

The NHS improvement plan for psychological care after stroke (NHS, 2011) specifies that psychological care after stroke should be multi-faceted and be considered an integral aspect of recovery at a multi-disciplinary level. The National Stroke Strategy (DoH, 2007) also emphasises the importance of a psychological pathway of rehabilitation which addresses the psychological changes that can occur following a stroke. Such policies have largely focused on 
the need to address the psychological difficulties which can follow stroke (e.g. anxiety, depression) and how such difficulties can impact on rehabilitation and recovery. The findings in this study show that the phenomenon of PTG can occur in a population of stroke survivors and this has implications for how psychological wellbeing post-stroke is conceptualised. More specifically, this study shows that PTG can be a psychological outcome for some stroke survivors and highlights that clinical perspectives could be broadened to include the possibility of growth following stroke.

From an ethical standpoint, caution is required regarding the potential use of PTG as an outcome measure in a clinical setting. Using PTG as an outcome measure would risk PTG becoming a standard against which progress is measured and interfere with the organic meaning-making process post-stroke. Nevertheless, the findings in this study support of use of PTG outlined by Joseph and Linley (2006), some of which include: integrating PTG into clinical practice through increasing awareness of the potential for PTG, listening out for news of growth when working with stroke survivors and using reflective listening skills to focus on narratives of PTG during therapy. There is also emerging evidence to suggest that peer support can facilitate the development of PTG in comparable trauma survivors (e.g. Morris et al. 2011). Using a sample of breast cancer survivors, Morris et al. (2011) found that accessing a peer support programme promoted the development of PTG.

Moreover, the findings in this study warrant consideration in clinical settings, particularly regarding the possibility of psychological growth following trauma in adults who are approximately 65 years of age and over. The mean age of participants in this study is somewhat older than studies which typically examine PTG in trauma survivors which suggests that growth can occur across the lifespan. It is possible that the finding that denial coping at time 1 was associated with rumination at time 2 may be unique to the population sampled. This finding has clinical implications in terms of how clinicians conceptualise the potential repertoires of response post-trauma in cohorts similar to the sample in this study. Further research is needed to expand our understanding regarding the complex process of psychological adjustment post-stroke in older people.

The literature on positive psychology suggests that increased happiness confers many benefits other than feeling good, some of which include improved health, success and social 
engagement (Lyubomirsky et al. 2005). There is also increasing evidence to support the efficacy of positive psychology interventions such as writing three good things every day, practicing gratitude and identifying personal strength in reducing feelings of depression (Seligman et al. 2005). It is unknown whether positive psychological interventions can facilitate the development of PTG. According to the model of PTG proposed by Tedeschi and Calhoun (2004), rather than positive interventions facilitating PTG, a process of deliberate rumination mediates the effect of factors such as social support on PTG. Studies have found that providing social support may be more beneficial than receiving it in a study of older couples (Brown et al. 2003). Interestingly, this finding has also been replicated in stroke survivors (Morris \& Morris, 2012). It is likely that providing social support is associated with aspects of PTG such as altruism and increased compassion for others. This has implications for how group therapy interventions are evaluated and also in understanding the psychological processes underpinning peer support in stroke survivors. It also highlights the importance of using post-stroke psychological interventions which are grounded in an empirical evidence-base.

Finally, it is important to consider the clinical implications of this study within the context of wider systemic factors such as culture and organisational systems. Cultural factors determine how potentially traumatic experiences are defined and what determines adaptive and maladaptive functioning (Wilson \& So-Kum Tang, 2007). In this way, it is important to consider the interaction between PTG and cultural beliefs pertaining to trauma and recovery in the context of different services and settings. By way of example, a cultural bias in a society toward being strong and stoic when faced with adversity may place a burden on a stroke survivor to feel that they ought to be experiencing PTG. Similarly, a service which evaluates clinical outcomes on the basis of PTG may motivate service users and professionals alike to perceive greater changes or PTG.

\subsection{Recommendations for future research}

The findings of this study provide preliminary support that PTG can occur in a sample of stroke survivors. Nevertheless, the literature on PTG in stroke survivors is limited and further research is required to determine whether these findings can be replicated. A strength of this 
study was the use of a longitudinal design, but due to the limited scope of this study stroke survivors were only studied in the acute stages post-stroke. In this way, further research is required to investigate the development of PTG over time (i.e. from the acute phases to several years post-stroke) and to determine the relative role of automatic and deliberate rumination over time. Elsewhere in the literature, there is emerging evidence which highlights the role of deliberate rumination in predicting of PTG (Hallam \& Morris, 2014). Such findings provide empirical support for the model of PTG proposed by Tedeschi and Calhoun (1995, 2004) which emphasises the role of deliberate, effortful rumination in the development of PTG.

Future research could employ alternative methodologies such as a control group to improve internal reliability. However, factors such as life-stage factors, previous experience of trauma and concurrent health difficulties need to be carefully considered and accounted for in explaining PTG. In the case of longitudinal studies, it is likely that intervening factors (e.g. life event) impact on the potential for PTG. Conducting studies with greater power would improve the magnitude of effects.

Regression analyses revealed that the data in this study did not fit neatly with the model of PTG proposed by Schaefer and Moos (1992) or Tedeschi \& Calhoun $(1995,2004)$. Instead, this study found that active coping, age and rumination were the greatest predictors of PTG. This study also found that the relationship between PTSS and PTG was mediated by rumination over time. This finding broadly supports the model of PTG proposed by Tedeschi and Calhoun (2004), but further work is required to understand the precise role of variables such as PTSS and denial coping in the development of PTG. There also appears to be a gap in the literature in terms of the measurement of positive emotions which are theorised to be linked to coping successes and are likely to manifest in the transformative phase associated with PTG.

\subsection{Conclusions}

Increasingly, it is recognised that exposure to traumatic events can lead to transformational changes and what is termed posttraumatic growth (PTG). A stroke bears many of the hallmarks of a traumatic event in that it can threaten one's sense of mortality and also, given 
that it is an acute event which can have lasting consequences such as complex disability. Models of PTG have been developed in an attempt to account for the process of transformational change inherent in PTG (e.g. Schaefer \& Moos, 1992; Tedeschi \& Calhoun, 1995, 2004).

Using a sample of stroke survivors, this study provides evidence that PTG can occur in stroke survivors and that such growth extends over time post-stroke. Reported levels of PTG in stroke survivors appear to be similar to those of trauma survivors who have been exposed to similar traumas (e.g. cancer). This study provides important evidence that rumination or cognitive processing mediates the relationship between PTSS and PTG over time. Such findings broadly support the model of PTG proposed by Tedeschi and Calhoun $(1995,2004)$ and have important implications for how PTG is conceptualised in the acute phase posttrauma and longitudinally. In addition, this study also lends support to Tedeschi and Calhoun's (2004) proposal that a struggle with trauma which involves the experience of psychological distress, attempts at coping and rumination is necessary in order for PTG to occur.

This study found that active coping, rumination and age were most predictive of PTG at time 2. Correlational findings revealed that stroke survivors who felt socially supported at up to 11 months post-stroke were more likely to experience PTG. Of note, active coping did not mediate the relationship between social support and PTG. Further examination of the complex interplay of factors such as active coping, denial coping, PTSS and rumination in predicting PTG is required to increase our understanding regarding the relative role of proximate and distal factors in predicting PTG. 


\section{References}

Abraido-Lanza, A.F., Guier, C. \& Colon, R.M. (1998). Psychological thriving among Latinas with chronic illness. Journal of Social Issues, 54, 405-424.

Adamson, J., Beswick, A. \& Ebrahim, S. (2004). Is stroke the most common cause of disability? Journal of Stroke and Cerebrovascular Diseases, 13(4), 171-177

Affleck, G., Tennen, H., Croog, S. \& Levine, S. (1987). Causal attribution, perceived benefits, and morbidity following a heart attack. Journal of Consulting and Clinical Psychology, $55,29-35$.

Aguinis, H., Gottfredson, R.K. \& Joo, H. (2013). Best-practice recommendations for defining, identifying and handling outliers. Organisational Research Methods, 16 (2), 270-301.

Ai, A.L., Tice, T.N., Whitsett, D., Ishisaka, T., \& Chim, M. (2007). Posttraumatic symptoms and growth of Kosovar war refugees: The influence of hope and cognitive coping. The Journal of Positive Psychology, 2(1), 55-65.

Aldwin, C.M. \& Levenson, M.R. (2004). Posttraumatic growth: A developmental perspective. Psychological Inquiry, 15(1), 9-22.

American Psychiatric Association (APA). (1994). Diagnostic and statistical manual of mental disorders (3rd edn.). Washington, DC: APA.

Armeli, S., Gunthert, K. C. \& Cohen, L. H. (2001). Stressor appraisals, coping, and post-event outcomes: The dimensionality and antecedents of stress-related growth. Journal of Social and Clinical Psychology, 20(3), 366-395.

Ayerbe, L., Ayis, S., Wolfe, C. D. \& Rudd, A. G. (2013). Natural history, predictors and of depression after stroke: systematic review and meta-analysis. The British Journal of Psychiatry, 202(1), 14-21.

Babbie, E. (1990). Survey research methods (2nd ed). Calafornia: Wadsworth Publishing. 
Bakker, M. \& Wicherts, J.M. (2014). Outlier Removal and the Relation with Reporting Errors and Quality of Psychological Research. PLOS ONE, 9(7). Online publication. doi:10.1371/journal.pone.0103360.

Baron, R.M., \& Kenny, D.A. (1986). The moderator-mediator variable distinction in social psychological research: Conceptual, strategic and statistical considerations. Journal of Personality and Social Psychology, 51, 1173-1182.

Bellizzi, K.M. \& Blank, T.O. (2006). Predicting posttraumatic growth in breast cancer survivors. Health Psychology, 25(1), 47-56.

Bitsch, L.J., Elklit, A. \& Christiansen, D.M. (2011). Basic problems with the measurement of posttraumatic growth. Odense: Syddansk Universitet.

Bland, J. M. \& Altman, D. G. (1996). Measurement error. British Medical Journal, 313, 744.

Bluvstein, I., Moravchick, L., Sheps, D., Schreiber, S. \& Bloch, M. (2012). Posttraumatic growth, posttraumatic stress symptoms and mental health among coronary heart disease survivors. Journal of Clinical Psychology in Medical Settings, 20(2), 164-172.

Briere, J. \& Elliott, D.M. (2003). Prevalence and psychological sequelae of self-reported childhood physical and sexual abuse in a general population sample of men and women. Child abuse \& neglect, 27(10), 1205-1222.

Brown, R.D., Whisnant, J.P., Sicks, J.D., O'Fallon, W.M. \& Wiebers, D.O. (1996). Stroke incidence, prevalence, and survival: secular trends in Rochester, Minnesota, through 1989. Stroke; a journal of cerebral circulation, 27(3), 373-380.

Bruggimann, L., Annoni, J. M., Staub, F., Von Steinbüchel, N., Van der Linden, M. \& Bogousslavsky, J. (2006). Chronic posttraumatic stress symptoms after nonsevere stroke. Neurology, 66(4), 513-516.

Burn, J., Dennis, M., Bamford, J., Sandercock, P., Wade, D. et al. (1994). Long-term risk of recurrent stroke after a first-ever stroke. The Oxfordshire Community Stroke Project. Stroke, 25(2), 333-337. 
Burton, C. R. (2000). Living with stroke: a phenomenological study. Journal of advanced nursing, 32(2), 301-309.

Calhoun, L.G., Cann, A. \& Tedeschi, R.G. (2010). The posttraumatic growth model: Sociocultural considerations. Posttraumatic growth and culturally competent practice: Lessons learned from around the globe, 1-14. Retrieved $14^{\text {th }}$ January from http://onlinelibrary.wiley.com/doi/10.1002/9781118270028.ch1/summary.

Calhoun, L.G., Cann, A., Tedeschi, R.G. \& McMillan, J. (2000). A correlational test of the relationship between posttraumatic growth, religion, and cognitive processing. Journal of traumatic stress, 13(3), 521-527.

Calhoun, L.G. \& Tedeschi, R.G. (1998). Beyond recovery from trauma: Implications for clinical practice and research. Journal of Social Issues, 54(2), 357-371.

Calhoun, L.G., \& Tedeschi, R.G. (1991). Perceiving benefits in traumatic events: Some issues for practicing psychologists. The Journal of Training and Practice in Professional Psychology, 5, 45-52.

Campbell, W.K., Brunell, A.B. \& Foster, J.D. (2004). Sitting here in limbo: Ego shock and posttraumatic growth. Psychological Inquiry, 15(1), 22-26.

Campbell Burton, C., Murray, J., Holmes, J., Astin, F., Greenwood, D. \& Knapp, P. (2013). Frequency of anxiety after stroke: a systematic review and meta-analysis of observational studies. International Journal of Stroke, 8(7), 545-559.

Cann, A., Calhoun, L.G., Tedeschi, R.G., Triplett, K.N., Vishnevsky, T. \& Lindstrom, C.M. (2011). Assessing posttraumatic cognitive processes: The event related rumination inventory. Anxiety, Stress, \& Coping, 24(2), 137-156.

Caplan, G. (1964). Principles of preventive psychiatry. Oxford, England: Basic Books. Carboon, I., Anderson, V.A., Pollard, A., Szer, J. \& Seymour, J.F. (2005). Posttraumatic growth following a cancer diagnosis: Do world assumptions contribute? Traumatology, $11(4), 269-283$. 
Carter, J. W., \& Carter, M. (1989). Sweet grapes: How to stop being infertile and start living again. Indianapolis, US: Perspectives Press.

Carver, C. S., Scheier, M. F. \& Weintraub, J. K. (1989). Assessing coping strategies: a theoretically based approach. Journal of personality and social psychology, 56(2), 267-283.

Chan, K. \& Bhandari, M. (2011). Three-minute critical appraisal of a case series article. Indian Journal of Orthopaedics, 45, 103-104.

Chan, M. W., Ho, S. M., Tedeschi, R. G. \& Leung, C. W. (2011). The valence of attentional bias and cancer-related rumination in posttraumatic stress and posttraumatic growth among women with breast cancer. Psycho-Oncology, 20(5), 544-552.

Cohen, J. (1988). Statistical power analysis for the behavioral sciences (2nd ed.). New Jersey, US: Erlbaum.

Collin, C., Wade, D.T., Davies, S. \& Horne, V. (1988). The Barthel ADL Index: a reliability study. Disability \& Rehabilitation, 10(2), 61-63.

Cordova, M.J., Cunningham, L.L., Carlson, C.R. \& Andrykowski, M.A. (2001). Posttraumatic growth following breast cancer: a controlled comparison study. Health Psychology, 20(3), 176-185.

Cox, A. M., McKevitt, C., Rudd, A. G. \& Wolfe, C. D. (2006). Socioeconomic status and stroke. The Lancet Neurology, 5(2), 181-188.

Creamer, M., McFarlane, A.C. \& Burgess, P. (2005). Psychopathology following trauma: The role of subjective experience. Journal of affective disorders, 86(2), 175-182.

Danhauer, S. C., Russell, G. B., Tedeschi, R. G., Jesse, M. T., Vishnevsky, T., Daley et al. (2013). A Longitudinal Investigation of Posttraumatic Growth in Adult Patients Undergoing Treatment for Acute Leukemia. Journal of Clinical Psychology in Medical Settings, 20(1), 13-24.

Dekel, S., Ein-Dor, T. \& Solomon, Z. (2012). Posttraumatic growth and posttraumatic 
distress: A longitudinal study. Psychological Trauma: Theory, Research, Practice, and Policy, 4, 94-101.

Dekel, S., Mandl, C. \& Solomon, Z. (2011). Shared and unique predictors of post-traumatic growth and distress. Journal of Clinical Psychology, 67(3), 241-252.

Department of Health (2007) National stroke strategy [Electronic version]. London: Department of Health.

Duffy, R. D., Allan, B. A., Autin, K. L., \& Bott, E. M. (2013). Calling and life satisfaction: It's not about having it, it's about living it. Journal of Counseling Psychology, 60(1), 42-52.

Efron, B. \& Tibshirani, R. (1993). An Introduction to the Bootstrap, Monographs on Statistics and Applied Probability. New York: Chapman \& Hall.

Ehring, T. \& Ehlers, A. (2014). Does rumination mediate the relationship between emotion regulation ability and posttraumatic stress disorder? European journal of psychotraumatology, 5. Doi:10.3402/ejpt.v5.23547

Erceg-Hurn, D.M. \& Mirosevich, V.M. (2008). Modern robust statistical methods: an easy way to maximize the accuracy and power of your research. American Psychologist, 63(7), 591-601.

Faul, F., Erdfelder, E., Lang, A.G. \& Buchner, A. (2007). G*Power 3: A flexible statistical power analysis program for the social, behavioral, and biomedical sciences. Behavior Research Methods, 39, 175-191.

Feigin, V.L., Forouzanfar, M.H., Krishnamurthi, R., Mensah, G.A., Connor, M., et al. (2014). Global and regional burden of stroke during 1990-2010: findings from the Global Burden of Disease Study 2010. Lancet, 383(9913), 245-254.

Field, A. (2013). Discovering statistics using IBM SPSS statistics ( $4^{\text {th }}$ ed.). London: Sage.

Field, E. L., Norman, P. \& Barton, J. (2008). Cross-sectional and prospective associations between cognitive appraisals and posttraumatic stress disorder symptoms following stroke. Behaviour research and therapy, 46(1), 62-70. 
Fitzmaurice, G. M. \& Ravichandran, C. (2008). A primer in longitudinal data analysis. Circulation, 118(19), 2005-2010.

Fontana, A. \& Rosenheck, R. (1998). Psychological benefits and liabilities of traumatic exposure in the war zone. Journal of Traumatic Stress, 11(3), 485-503.

Frankl V.E. (1963). Man's search for meaning. New York: Washington Square Press.

Frazier, P., Conlon, A. \& Glaser, T. (2001). Positive and negative life changes following sexual assault. Journal of consulting and clinical psychology, 69(6), 1048.

Frazier, P.A. \& Kaler, M.E. (2006). Assessing the validity of self-reported stress-related growth. Journal of Consulting and Clinical Psychology, 74(5), 859-869.

Fredrickson, B.L. (2001). The role of positive emotions in positive psychology: The broadenand-build theory of positive emotions. American psychologist, 56(3), 218-226.

Fredrickson, B.L., Tugade, M.M., Waugh, C.E. \& Larkin, G.R. (2003). What good are positive emotions in crisis? A prospective study of resilience and emotions following the terrorist attacks on the United States on September 11th, 2001. Journal of personality and social psychology, 84(2), 365-376.

Furie, K.L., Kasner, S.E., Adams, R.J., Albers, G.W., Bush, R.L. et al. (2011). Guidelines for the prevention of stroke in patients with stroke or transient ischemic attack a guideline for healthcare professionals from the American Heart Association/American Stroke Association. Stroke, 42(1), 227-276.

Gable, S.L. \& Haidt, J. (2005). What (and why) is positive psychology? Review of general psychology, 9(2), 103-110.

Gangstad, B., Norman, P. \& Barton, J. (2009). Cognitive processing and posttraumatic growth after stroke. Rehabilitation Psychology, 54(1), 69-75.

Garnefski, N., Kraaij, V., Schroevers, M.J. \& Somsen, G.A. (2008). Post-traumatic growth after a myocardial infarction: a matter of personality, psychological health, or cognitive coping? Journal of Clinical Psychology in Medical Settings, 15(4), 270-277. 
Gerald, J. H. \& George, S. H. (2010). Self-report: Psychology's four-letter word. American Journal of Psychology, 123(2), 181-188.

Giles, M.F. \& Rothwell, P.M. (2007). Risk of stroke early after transient ischaemic attack: a systematic review and meta-analysis. The Lancet Neurology, 6(12), 1063-1072.

Gillen, G. (2005). Positive consequences of surviving a stroke. The American Journal of Occupational Therapy, 59(3), 346-350.

Glass, T.A. \& Maddox, G.L. (1992). The quality and quantity of social support: stroke recovery as psycho-social transition. Social science \& medicine, 34(11), 1249-1261.

Goodhart, D. E. (1985). Some psychological effects associated with positive and negative thinking about stressful event outcomes: was Pollyanna right? Journal of Personality and Social Psychology, 48(1), 216-232.

Hackett, M.L., Yapa, C., Parag, V. \& Anderson, C.S. (2005). Frequency of depression after stroke a systematic review of observational studies. Stroke, 36(6), 1330-1340.

Hadidi, N., Buckwalter, K., Lindquist, R. \& Rangen, C. (2012). Lessons learned in recruitment and retention of stroke survivors. Journal of Neuroscience Nursing, 44(2), 105-110.

Hallam, W., \& Morris, R. (2014). Post-traumatic growth in stroke carers: A comparison of theories. British journal of health psychology, 19(3), 619-635.

Hanson, W. E., Creswell, J. W., Clark, V. L. P., Petska, K. S. \& Creswell, J. D. (2005). Mixed methods research designs in counseling psychology. Journal of counseling psychology, 52(2), 224.

Hare, R., Rogers, H., Lester, H., McManus, R. J., \& Mant, J. (2006). What do stroke patients and their carers want from community services? Family Practice, 23(1), 131-136.

Harvey J.H., Barnett K. \& Overstreet A. (2004). Trauma growth and other outcomes attendant to loss. Psychological Inquiry, 15, 26-29.

Hayes, A.F. (2013). Introduction to mediation, moderation, and conditional process analysis: A regression-based approach. London: Guilford Press. 
Hayes, A.F. (2009). Beyond Baron and Kenny: Statistical mediation analysis in the new millennium. Communication monographs, 76(4), 408-420.

Hefferon, K., Grealy, M. \& Mutrie, N. (2009). Post-traumatic growth and life threatening physical illness: A systematic review of the qualitative literature. British journal of health psychology, 14(2), 343-378.

Helgeson, V.S., Reynolds, K.A. \& Tomich, P.L. (2006). A meta-analytic review of benefit finding and growth. Journal of consulting and clinical psychology, 74(5), 797-816.

Herbert, T.B. \& Cohen, S. (1993). Depression and immunity: a meta-analytic review. Psychological bulletin, 113(3), 472.

Hillis, A. E. \& Tippett, D. C. (2014). Stroke Recovery: Surprising Influences and Residual Consequences. Advances in Medicine, 378263. doi:10.1155/2014/378263

Hogan, N. S. \& Schmidt, L. A. (2002). Testing the grief to personal growth model using structural equation modeling. Death Studies, 26(8), 615-634.

Horowitz, M., Wilner, N. \& Alvarez, W. (1979). Impact of Event Scale: a measure of subjective stress. Psychosomatic medicine, 41(3), 209-218.

House, A., Dennis, M., Mogridge, L., Warlow, C., Hawton, K. E. I. T. H. \& Jones, L. E. S. L. E. Y. (1991). Mood disorders in the year after first stroke. The British Journal of Psychiatry, 158(1), 83-92.

Ingram, R.E. \& Price, J.M. (2001). The role of vulnerability in understanding psychopathology. In R. E. Ingram \& J. M. Price (Eds.), Vulnerability to psychopathology: Risk across the lifespan (pp. 3-19). New York: The Guilford Press. Janoff-Bulman, R. (2004). Posttraumatic growth: Three explanatory models. Psychological Inquiry, 15(1), 30-34.

Janoff-Bulman, R. (1992). Shattered assumptions: Towards a new psychology of trauma. New York: Free Press.

Joseph, S. \& Linley, P.A. (2006). Growth following adversity: Theoretical perspectives and 
implications for clinical practice. Clinical psychology review, 26(8), 1041-1053.

Kaufman, S. R. \& Becker, G. (1991). Content and boundaries of medicine in long-term care: physicians talk about stroke. The Gerontologist, 31(2), 238-245.

Keselman, H. J., Algina, J., Lix, L. M., Wilcox, R. R. \& Deering, K. N. (2008). A generally robust approach for testing hypotheses and setting confidence intervals for effect sizes. Psychological Methods, 13(2), 110-129.

Kierkegaard, S. (1983). Fear and trembling. New Jersey, US: Princeton University Press. Kuenemund, A., Zwick, S., Rief, W. \& Exner, C. (2014). (Re-) defining the self-Enhanced posttraumatic growth and event centrality in stroke survivors: A mixed-method approach and control comparison study. Journal of health psychology. Advance online publicaiton. doi: 1359105314535457.

Lazzeroni, L.C. \& Ray, A. (2012). The cost of large numbers of hypothesis tests on power, effect size and sample size. Molecular Psychiatry, 17, 108-114

Lazarus, R.S., \& Folkman, S. (1986). Cognitive theories of stress and the issue of circularity. In Dynamics of stress (pp. 63-80). U.S: Springer.

Lehman, D.R., Ellard, J.H. \& Wortman, C.B. (1986). Social support for the bereaved: Recipients' and providers' perspectives on what is helpful. Journal of consulting and clinical psychology, 54(4), 438-446.

Lelorain, S., Bonnaud-Antignac, A. \& Florin, A. (2010). Long term posttraumatic growth after breast cancer: prevalence, predictors and relationships with psychological health. Journal of Clinical Psychology in Medical Settings, 17(1), 14-22.

Linley, P. A. \& Joseph, S. (2004). Positive change following trauma and adversity: A review. Journal of traumatic stress, 17(1), 11-21.

Litman, J.A. (2006). The COPE inventory: Dimensionality and relationships with approachand avoidance-motives and positive and negative traits. Personality and Individual Differences, 41(2), 273-284. 
Lockwood, C. M. \& MacKinnon, D. P. (1998, March). Bootstrapping the standard error of the mediated effect. In Proceedings of the 23rd annual meeting of SAS Users Group International (pp. 997-1002).

Luszczynska, A., Durawa, A.B., Dudzinska, M., Kwiatkowska, M., Knysz, B. et al. (2012). The effects of mortality reminders on posttraumatic growth and finding benefits among patients with life-threatening illness and their caregivers. Psychology \& Health, 27, $1227-1243$.

Lyubomirsky, S., King, L. \& Diener, E. (2005). The benefits of frequent positive affect: does happiness lead to success? Psychological bulletin, 131(6), 803-855

Maercker, A. \& Langner, R. (2001). Posttraumatic personal growth: Validation of German versions of 2 questionnaires. Diagnostica, 47, 153-162.

Maercker, A. \& Zoellner, T. (2004). The Janus face of self-perceived growth: Toward a twocomponent model of posttraumatic growth. Psychological Inquiry, 15, 41-48.

Mahoney, F.I. (1965). Functional evaluation: the Barthel index. Maryland state medical journal, 14, 61-65.

Martin, L.L., \& Tesser, A. (1996). Some ruminative thoughts. Advances in social cognition, 9, 1-47.

McFarland, C. \& Alvaro, C. (2000). The impact of motivation on temporal comparisons: coping with traumatic events by perceiving personal growth. Journal of personality and social psychology, 79(3), 327-343.

McMillen, J.C., Smith, E.M. \& Fisher, R.H. (1997). Perceived benefit and mental health after three types of disaster. Journal of consulting and clinical psychology, 65(5), 733-739.

McMillen, J.C. \& Cook, C.L. (2003). The positive by-products of spinal cord injury and their correlates. Rehabilitation Psychology, 48(2), 77-85.

Menard, S. (2007). Handbook of longitudinal research: design, measurement, and analysis. London: Elsevier. 
Merriman, C., Norman, P. \& Barton, J. (2007). Psychological correlates of PTSD symptoms following stroke. Psychology. Health and Medicine, 12(5), 592-602.

Micceri, T. (1989). The unicorn, the normal curve, and other improbable creatures. Psychological Bulletin, 105(1), 156-166.

Mohan, K. M., Wolfe, C. D., Rudd, A. G., Heuschmann, P. U., Kolominsky-Rabas, P. L. \& Grieve, A. P. (2011). Risk and cumulative risk of stroke recurrence a systematic review and meta-analysis. Stroke, 42(5), 1489-1494.

Morrill, E. F., Brewer, N. T., O'Neill, S. C., Lillie, S. E., Dees, E. C., Carey, L. A. et al. (2008). The interaction of post-traumatic growth and post-traumatic stress symptoms in predicting depressive symptoms and quality of life. Psycho-Oncology, 17(9), 948-953.

Morris, B. A., Campbell, M., Dwyer, M., Dunn, J. \& Chambers, S. K. (2011). Survivor identity and post-traumatic growth after participating in challenge-based peer-support programmes. British journal of health psychology, 16(3), 660-674.

Morris, R. \& Morris, P. (2012). Participants' experiences of hospital-based peer support groups for stroke patients and carers. Disability and rehabilitation, 34(4), 347-354.

Morris, B.A., Shakespeare-Finch, J. \& Scott, J.L. (2007). Coping processes and dimensions of posttraumatic growth. The Australasian Journal of Disaster and Trauma Studies, 112.

Morrison, V., Pollard, B., Johnston, M. \& MacWalter, R. (2005). Anxiety and depression 3 years following stroke: demographic, clinical, and psychological predictors. Journal of psychosomatic research, 59(4), 209-213.

Murray, J., Young, J., Forster, A. \& Ashworth, R. (2003). Developing a primary care-based stroke model: the prevalence of longer-term problems experienced by patients and carers. British Journal of General Practice, 53(495), 803-807.

National Audit Office. (2005). Reducing Brain Damage: Faster access to better stroke care. HC452. London: The Stationary Office. 
Neimeyer, R.A., Baldwin, S.A. \& Gillies, J. (2006). Continuing bonds and reconstructing meaning: Mitigating complications in bereavement. Death studies, 30(8), 715-738.

NHS improvement (2011). Psychological care after stroke: improving stroke services for people with cognitive and mood disorders. Retrieved January $13^{\text {th }} 2015$ from https://www.nice.org.uk/media/default/sharedlearning/531 strokepsychologicalsupportfinal.pdf

Nicholls, R. A. (1955). Nietzsche in the early work of Thomas Mann (Vol. 45). University of California Press.

Nisbett, R. E. \& Wilson, T. D. (1977). Telling more than we can know: Verbal reports on mental processes. Psychological review, 84(3), 231-260.

Nolen-Hoeksema, S. \& Davis, C.G. (2004). Theoretical and methodological issues in the assessment and interpretation of posttraumatic growth. Psychological Inquiry, 15(1), 60-64.

Nolen-Hoeksema, S., Larson, J. \& Grayson, C. (1999). Explaining the gender difference in depressive symptoms. Journal of personality and social psychology, 77(5), 1061-1072.

Office of National Statistics (2014). Statistical bulletin. Retrieved on $16^{\text {th }}$ of January: http://www.ons.gov.uk/ons/dcp171778_370815.pdf

Orne M.T. (1962). On the social psychology of the psychological experiment: With particular reference to demand characteristics and their implications. American Psychologist, 17, 776-783.

Osborne, J.W. (2013). Best practices in data cleaning. UK: Sage Publications, Inc.

Ostir, G.V., Markides, K.S., Peek, M.K. \& Goodwin, J.S. (2001). The association between emotional well-being and the incidence of stroke in older adults. Psychosomatic medicine, 63(2), 210-215.

Panagopoulou, E., Triantafyllou, A., Mitziori, G. \& Benos, A. (2009). Dyadic benefit finding after myocardial infarction: A qualitative investigation. Heart and Lung, 38(4), 292- 
297.

Park, C. L. (2010). Making sense of the meaning literature: an integrative review of meaning making and its effects on adjustment to stressful life events. Psychological bulletin, 136(2), 257-301.

Park, C. L. (2004). The notion of growth following stressful life experiences: Problems and prospects. Psychological Inquiry, 15, 69-76.

Park, C.L., Cohen, L.H. \& Murch, R.L. (1996). Assessment and prediction of stress-related growth. Journal of Personality, 64, 71- 105.

Park, C.L., \& Folkman, S. (1997). Meaning in the context of stress and coping. Review of general psychology, 1(2), 115-144.

Park, C.L., \& Helgeson, V. (2006). Introduction to the special section: growth following highly stressful life events--current status and future directions. Journal of consulting and clinical psychology, 74(5), 791-796.

Phelps, L.F., Williams, R.M., Raichle, K.A., Turner, A.P. \& Ehde, D.M. (2008). The importance of cognitive processing to adjustment in the 1st year following amputation. Rehabilitation Psychology, 53(1), 28-38.

Pollack, M. R. \& Disler, P. B. (2002). 2: Rehabilitation of patients after stroke. The Medical Journal of Australia, 177(8), 452-456.

Polkinghorne, D. E. (2005). Language and meaning: Data collection in qualitative research. Journal of counseling psychology, 52(2), 137-145

Pollard, C. \& Kennedy, P. (2007). A longitudinal analysis of emotional impact, coping strategies and post-traumatic psychological growth following spinal cord injury: A 10-year review. British journal of health psychology, 12(3), 347-362.

Prati, G. \& Pietrantoni, L. (2009). Optimism, social support, and coping strategies as factors contributing to posttraumatic growth: A meta-analysis. Journal of Loss and Trauma, 14(5), 364-388. 
Preacher, K. J. \& Hayes, A. F. (2008). Asymptotic and resampling strategies for assessing and comparing indirect effects in multiple mediator models. Behavior research methods, 40(3), 879-891.

Richman, L. S., Kubzansky, L., Maselko, J., Kawachi, I., Choo, P. \& Bauer, M. (2005). Positive emotion and health: going beyond the negative. Health Psychology, 24(4), 422.

Rochowicz, J. A. (2010). Bootstrapping analysis, inferential statistics and EXCEL. Spreadsheets in Education, 4(3). Retrieved on $4^{\text {th }}$ February from http://epublications.bond.edu.au/ejsie/vol4/iss3/4

Rosenthal, R. and Gaito, J. (1963). The interpretation of levels of significance by psychological researchers. Journal of Psychology, 55, 33-38.

Royal college of physicians (2012). National clinical guideline for stroke, 4th edition. London: RCP.

Rutter, C. M. \& Miglioretti, D. L. (2003). Estimating the accuracy of psychological scales using longitudinal data. Biostatistics, 4(1), 97-107.

Saka, Ö., McGuire, A. \& Wolfe, C. (2009). Cost of stroke in the United Kingdom. Age and ageing, 38(1), 27-32.

Schaefer, J.A. \& Moos, R.H. (1992). Life crises and personal growth. In Carpenter, B. N. (Ed.), Personal coping: Theory, research and application (pp.149-170). US: Greenwood publishing.

Schaefer, J. A. \& Moos, R. H. (1998). The context for posttraumatic growth: Life crises, individual and social resources, and coping. In R. G. Tedeschi, C. L. Park, \& L. G. Calhoun (Eds.), Posttraumatic growth: Positive changes in the aftermath of crisis (pp. 99-126). Mahwah, NJ: Lawrence Erlbaum.

Schroevers, M. J., Helgeson, V. S., Sanderman, R. \& Ranchor, A. V. (2010). Type of social support matters for prediction of posttraumatic growth among cancer survivors. Psycho-Oncology, 19(1), 46-53. 
Scrignaro, M., Barni, S. \& Magrin, M. E. (2011). The combined contribution of social support and coping strategies in predicting post-traumatic growth: a longitudinal study on cancer patients. Psycho-Oncology, 20(8), 823-831.

Sears, S. R., Stanton, A. L. \& Danoff-Burg, S. (2003). The yellow brick road and the emerald city: benefit finding, positive reappraisal coping and posttraumatic growth in women with early-stage breast cancer. Health Psychology, 22(5), 487-497.

Seligman, M.E. \& Csikszentmihalyi, M. (2000). Positive psychology: An introduction. American Psychologist, 55(1), 5-14.

Seligman, M. E., Steen, T. A., Park, N. \& Peterson, C. (2005). Positive psychology progress: empirical validation of interventions. American psychologist, 60(5), 410-421.

Sembi, S., Tarrier, N., O'Neill, P., Burns, A. \& Faragher, B. (1998). Does post-traumatic stress disorder occur after stroke: a preliminary study. International journal of geriatric, 13(5), 315-322.

Senol-Durak, E. \& Ayvasik, H. B. (2010). Factors associated with posttraumatic growth among myocardial infarction patients: Perceived social support, perception of the event and coping. Journal of clinical psychology in medical settings, 17(2), 150-158.

Seshadri, S., Beiser, A., Kelly-Hayes, M., Kase, C. S., Au, R. et al. (2006). The lifetime risk of stroke estimates from the Framingham Study. Stroke, 37(2), 345-350.

Shand, L. K., Cowlishaw, J. E., Brooker, J.E., Burney, S. \& Ricciardelli, L. A. (2015). Correlates of post-traumatic stress symptoms and growth in cancer patients: a systematic review and meta-analysis. Psycho-Oncology, 24. doi: 10.1002/pon.3719

Siegel, K., Schrimshaw, E.W., \& Pretter, S. (2005). Stress-related growth among women living with HIV/AIDS: Examination of an explanatory model. Journal of Behavioral Medicine, 28(5), 403-414.

Silva, S. M., Crespo, C. \& Canavarro, M. C. (2012). Pathways for psychological adjustment in breast cancer: A longitudinal study on coping strategies and posttraumatic 
growth. Psychology \& health, 27(11), 1323-1341.

Skilbeck, C. E., Wade, D. T., Hewer, R. L. \& Wood, V. A. (1983). Recovery after stroke. Journal of Neurology, Neurosurgery \& Psychiatry, 46(1), 5-8.

Smania, N., Gandolfi, M., Aglioti, S. M., Girardi, P., Fiaschi, A. \& Girardi, F. (2010). How long is the recovery of global aphasia? Twenty-five years of follow-up in a patient with left hemisphere stroke. Neurorehabilitation and neural repair, 24(9), 871-875.

Sobel, M.E. (1982). Asymptotic confidence intervals for indirect effects in structural equation models. Sociological methodology, 13, 290-312.

Somerfield, M.R. \& McCrae, R.R. (2000). Stress and coping research: Methodological challenges, theoretical advances, and clinical applications. American psychologist, 55(6), 620-625.

Splevins, K., Cohen, K., Bowley, J. \& Joseph, S. (2010). Theories of posttraumatic growth: Cross-cultural perspectives. Journal of Loss and Trauma, 15(3), 259-277.

Stanton, A. L., Bower, J. E. \& Low, C. A. (2006). Posttraumatic growth after cancer. Handbook of posttraumatic growth: Research and practice, 138-175.

Stanton, A. L., Danoff-burg, S. \& Huggins, M. E. (2002). The first year after breast cancer diagnosis: hope and coping strategies as predictors of adjustment. Psycho-Oncology, 11(2), 93-102.

Stone, J., Townend, E., Kwan, J., Haga, K., Dennis, M. S. \& Sharpe, M. (2004). Personality change after stroke: some preliminary observations. Journal of Neurology, Neurosurgery \& Psychiatry, 75(12), 1708-1713.

Stroebe, M. \& Schut, H. (1999). The dual process model of coping with bereavement: rationale and description. Death studies, (23), 197-224.

Stroke Association (2015). State of the Nation. Retrieved online on $5^{\text {th }}$ January from https://www.stroke.org.uk/sites/default/files/stroke_statistics_2015.pdf

Stroke Unit Trialists' Collaboration. (2007). Organised inpatient (stroke unit) care for stroke. 
Cochrane Database of Systematic Reviews, 4(4). Doi: 10.1002/14651858.CD000197

Specialist Unit for Review Evidence (SURE) 2013. Questions to assist with the critical appraisal of a systematic review. Available at:

http://www.cardiff.ac.uk/insrv/libraries/sure/doc/SURE_RCT_Checklist_2013.pdf

Specialist Unit for Review Evidence (SURE) 2013. Questions to assist with the critical appraisal of qualitative studies. Available at:

http://www.cardiff.ac.uk/insrv/libraries/sure/doc/SURE_RCT_Checklist_2013.pdf

Tabachnik, B.G., \& Fidell, L.S. (2007). Using multivariate statistics (5th ed.). Boston: Allyn \& Bacon.

Taku, K., Cann, A., Tedeschi, R. G. \& Calhoun, L. G. (2009). Intrusive versus deliberate rumination in posttraumatic growth across US and Japanese samples. Anxiety, Stress, \& Coping, 22(2), 129-136.

Tallman, B., Shaw, K., Schultz, J. \& Altmaier, E. (2010). Well-being and posttraumatic growth in unrelated donor marrow transplant survivors: a nine-year longitudinal study. Rehabilitation psychology, 55(2), 204-210.

Taylor, S. E., Kemeny, M. E., Reed, G. M., Bower, J. E., \& Gruenewald, T. L. (2000). Psychological resources, positive illusions, and health. American psychologist, 55(1), 99-109.

Tedeschi, R. G. \& Calhoun, L. G. (1995). Trauma and transformation: Growing in the aftermath of suffering. US: Sage Publications.

Tedeschi, R.G. \& Calhoun, L.G. (1996). The Posttraumatic Growth Inventory: Measuring the positive legacy of trauma. Journal of traumatic stress, 9(3), 455-471.

Tedeschi, R.G. \& Calhoun, L.G. (2004). Posttraumatic Growth: Conceptual Foundations and Empirical Evidence. Psychological inquiry, 15(1), 1-18.

Thompson, M. (2000). Life after rape: A chance to speak? Sexual and Relationship Therapy, 15(4), 325-343. 
Thorndike, E.L. (1920). A constant error in psychological ratings. Journal of Applied Psychology, 4, 25-29.

Tornstam, L. (1994). Gerotranscendence-a theoretical and empirical exploration. In: L.E. Thomas, \& S.A. Eisenhandler (Eds.), Aging and the religious dimension (pp. 203-225). Westport: Greenwood Publishing Group.

Townsend, N., Wickramasinghe, K., Bhatnagar, P., Smolina, K., Nichols, M. et al. (2012). Coronary heart disease statistics 2012 edition. London: British Heart Foundation.

Townsley, C.A., Selby, R. \& Siu, L.L. (2005). Systematic review of barriers to the recruitment of older patients with cancer onto clinical trials. Journal of Clinical Oncology, 23(13), 3112-3124.

Treynor, W., Gonzalez, R. \& Nolen-Hoeksema, S. (2003). Rumination reconsidered: A psychometric analysis. Cognitive Therapy and Research, 27(3), 247-259.

Updegraff, J. A., Taylor, S. E., Kemeny, M. E. \& Wyatt, G. E. (2002). Positive and negative effects of HIV-infection in women in low socioeconomic resources. Personality and Social Psychology Bulletin, 28, 382-394.

Wang, Y., Wang, H., Wang, J., Wu, J. \& Liu, X. (2013). Prevalence and predictors of posttraumatic growth in accidentally injured patients. Journal of clinical psychology in medical settings, 20(1), 3-12.

Weiss, T. (2002). Posttraumatic growth in women with breast cancer and their husbands: An intersubjective validation study. Journal of psychosocial Oncology, 20(2), 65-80.

Weiss, D.S. \& Marmar, C.R. (1997). The Impact of Event Scale-Revised. In J.P. Wilson, \& T.M. Keane (Eds.), Assessing Psychological Trauma and PTSD: A Practitioner's Handbook (pp. 399-411). New York: Guilford Press.

Wells, A. \& Sembi, S. (2004). Metacognitive focused therapy for PTSD: Effectiveness of a new treatment approach. Journal of Behavior Therapy and Experimental Psychiatry, 35, 307-318. 
West, T., \& Bernhardt, J. (2011). Physical activity in hospitalised stroke patients. Stroke Research and Treatment, 2012: doi:10.1155/2012/813765

WHO MONICA Project Investigators. (1988). The World Health Organization MONICA Project: Monitoring trends and determinants in cardiovascular disease. Journal of Clinical Epidemiology, 41, 105-114.

WHO. (2014). World health statistics 2014. Italy: WHO.

Widows, M. R., Jacobsen, P. B., Booth-Jones, M. \& Fields, K. K. (2005). Predictors of posttraumatic growth following bone marrow transplantation for cancer. Health psychology, 24(3), 266-273.

Wilcox, R.R. (2012). Introduction to robust estimation and hypothesis testing. London: Academic Press.

Williams, R. M., Davis, M. C. \& Millsap, R. E. (2002). Development of the cognitive processing of trauma scale. Clinical Psychology \& Psychotherapy, 9(5), 349-360.

Williams, J. \& MacKinnon, D. P. (2008). Resampling and distribution of the product methods for testing indirect effects in complex models. Structural Equation Modeling, 15(1), 23-51.

Wilson, J. P. \& So-Kum Tang, C.C. (2007). Cross-cultural assessment of psychological trauma and PTSD. UK: Springer.

Woike, B. \& Matic, D. (2004). Cognitive complexity in response to traumatic experiences. Journal of Personality, 72(3), 633-658.

Wortman, C.B. (2004). Posttraumatic growth: Progress and problems. Psychological Inquiry, 15(1), 81-90.

Wright, D. B., Kamala, L. \& Field, A. P. (2011). Using Bootstrap Estimation and the Plug-in Principle for Clinical Psychology Data. Journal of Experimental Psychopathology, 2, 252-270.

Yalom, I.D. (1980). Existential psychotherapy. US: Basic Books. 
Zimet, G.D., Dahlem, N. W., Zimet, S.G. \& Farley, G.K. (1988). The multidimensional scale of perceived social support. Journal of personality assessment, 52(1), 30-41.

Zoellner, T. \& Maercker, A. (2006). Posttraumatic growth in clinical psychology-A critical review and introduction of a two component model. Clinical psychology review, 26(5), 626-653.

Znoj, H. J., \& Keller, D. (2002). Mourning parents: Considering safeguards and their relation to health. Death studies, 26(7), 545-565. 
Appendix A Search terms for initial systematic review

Review question: What are the predictors of posttraumatic growth in stroke survivors?

The search terms below were combined using Boolean operators (i.e. 'and', 'or').

Search terms relating to posttraumatic growth were:

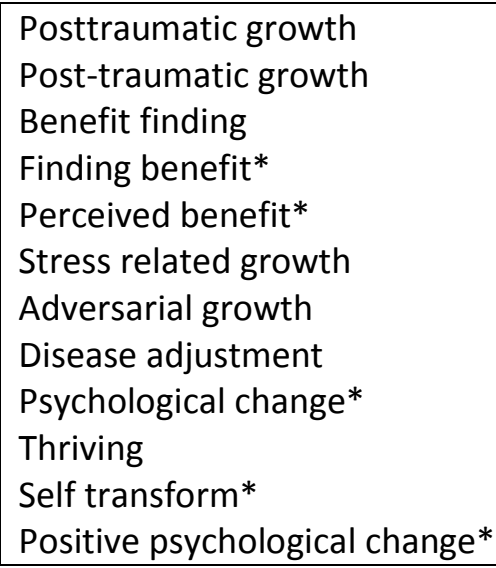

Search terms relating to stroke survivors were:

\begin{tabular}{|l}
\hline Patient* \\
Survivor* \\
Victim*
\end{tabular}

Search terms relating to stroke were:

\begin{tabular}{|l|}
\hline Stroke \\
CVA \\
Ischaemic stroke \\
Haemorrhagic stroke \\
Brain disorder \\
\hline
\end{tabular}


Appendix B Search terms for expanded systematic review

Review question: What are the predictors of posttraumatic growth in survivors of myocardial infarction?

The search terms below were combined using Boolean operators (i.e. 'and', 'or').

Search terms relating to posttraumatic growth were:

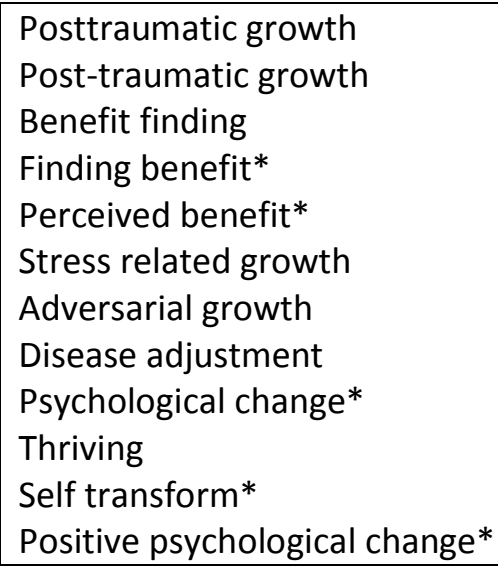

Search terms relating to stroke survivors were:

$$
\begin{array}{|l}
\hline \text { Patient* } \\
\text { Survivor* } \\
\text { Victim* }
\end{array}
$$

Search terms relating to myocardial infarction were:

Myocardial infarction
Heart attack
Ischaemic heart disease




\section{Participant Information Sheet}

\section{Study title: Predictors of post-traumatic growth in post-stroke survivors.}

We would like to invite you to take part in our research study. Before you decide, we would like you to understand why the research is being done and what it would involve for you. Please take time to read the following information carefully. The researcher is available to answer any questions you may have.

\section{What is the purpose of the study?}

This research looks at the experiences people have after a stroke, particularly how people cope and make sense of their stroke. Post-traumatic growth has been defined as a positive psychological change that can occur after a traumatic or challenging life experience. Some research suggests that people can experience post-traumatic growth after a stroke. This research aims to investigate how post-traumatic growth develops for people who have experienced a stroke.

\section{Why have I been invited?}

You have been invited to participate because you have experienced a stroke.

You can participate in this study if:

- You have experienced one stroke.

- You are over 18 years of age.

- You are not known to be pregnant.

\section{Do I have to take part?}

It is up to you to decide to join the study. The researcher will describe the study and go through this information sheet with you. If you agree to take part, we will then ask you to sign a consent form. You are free to withdraw at any time, without giving a reason. This would not affect the standard of care you receive. 


\section{What will happen to me if I take part?}

If you decide to take part, you will be asked to complete a set of questionnaires which take about thirty minutes to complete. You will complete the questionnaires with help from the researcher, in person, at a time that is convenient for you.

After 10 months, you will be contacted to repeat this set of questionnaires. This time, you will have the option to complete the questionnaires over the telephone. The researcher will send you a copy of the questionnaires by post so your response options are clear while on the telephone.

If you decide to take part, you will be asked whether you consent to the researcher accessing your medical notes and data. If you provide consent, the researcher will only access your medical notes for information about your stroke.

\section{What will I have to do?}

If you decide to take part, you will be asked to complete a set of questionnaires at an agreed time after your outpatient appointment with your stroke clinician. You will be contacted, by telephone, after 10 months to repeat the questionnaires.

\section{What are the possible disadvantages and risk of taking part?}

If you find completing the questionnaires raises issues that are distressing, you may find helpful information and insights on the stroke association website (http://www.stroke.org.uk/). But if you remain upset or concerned please contact the principal investigator, Grace Kelly at 02920870587 or the chief investigator, Professor Reg Morris at 02920870582.

\section{What are the possible benefits of taking part?}

We cannot promise the study will help you but the information we get from the study will help to increase the understanding of post-traumatic growth in the research literature.

\section{What if there is a problem?}

If you have a concern about any aspect of this study, you should ask to speak to the researchers who will do their best to answer your questions (contact Grace Kelly on 02920870582 or at kellyg7@cardiff.ac.uk).

If you remain unhappy and wish to complain formally you can do this through Cardiff University on 029 20879131 or at resgov@cardiff.ac.uk 


\section{Will my taking part in the study be kept confidential?}

All information which is collected about you during the course of the research will be kept strictly confidential, and any information about you which leaves the university will have your name and personal details removed so that you cannot be recognised.

Your participation in this research will not impact on the care you receive from other health professionals.

\section{What will happen if I don't carry on with the study?}

If you withdraw from the study all the identifiable information and data collected from you, to date, will be destroyed and your name removed from all study files.

\section{What will happen to the results of the research study?}

It is hoped that the results of the research will be published in a scientific journal. You will be given the opportunity to receive a summary of the findings after the research is complete. You will not be identified in any report/publication related to this research.

\section{Who is organising and funding the research?}

Cardiff University is sponsoring this research.

\section{Who has reviewed the study?}

All research in the NHS is looked at by an independent group of people, called a Research Ethics Committee, to protect your interests. This study has been reviewed and given favourable opinion by the South East Wales Research Ethics Committee.

\section{Further information and contact details.}

For further information about this study, please contact Grace Kelly (Researcher) on 02920870582 or at kellyg7@cardiff.ac.uk 
Letter of Invitation

\section{Dear sir/madam,}

I am writing to invite you to participate in a research project. The research project is looking at what people experience after they have had a stroke. I have attached an information sheet for you to consider; it explains what participation in this project would involve.

The researcher, Grace Kelly, is a trainee clinical psychologist who is conducting this research as part of her studies with Cardiff University. Grace will be available after your appointment with me, if you would like to discuss participating or if you have any questions.

Please note that your decision to participate, or not, will in no way impact on the treatment you receive with the Stroke team.

Yours sincerely,

*Insert stroke clinician name*

*Insert job title* 


\section{Participant Information Sheet}

Study title: Predictors of post-traumatic growth in post-stroke survivors.

We would like to invite you to take part in our research study. Before you decide, we would like you to understand why the research is being done and what it would involve for you. Please take time to read the following information carefully. The researcher is available to answer any questions you may have.

\section{What is the purpose of the study?}

This research looks at the experiences people have after a stroke, particularly how people cope and make sense of their stroke. Post-traumatic growth has been defined as a positive psychological change that can occur after a traumatic or challenging life experience. Some research suggests that people can experience post-traumatic growth after a stroke. This research aims to investigate how post-traumatic growth develops for people who have experienced a stroke.

\section{Why have I been invited?}

You have been invited to participate because you have experienced a stroke.

You can participate in this study if:

- You have experienced one stroke.

- You are over 18 years of age.

- You are not known to be pregnant.

\section{Do I have to take part?}

It is up to you to decide to join the study. The researcher will describe the study and go through this information sheet with you. If you agree to take part, we will then ask you to sign a consent form. You are free to withdraw at any time, without giving a reason. This would not affect the standard of care you receive. 


\section{What will happen to me if I take part?}

If you decide to take part, you will be asked to complete a set of questionnaires which take about thirty minutes to complete. You will complete the questionnaires with help from the researcher, in person, at a time that is convenient for you.

After 6 months, you will be contacted to repeat this set of questionnaires. This time, you will have the option to complete the questionnaires over the telephone. The researcher will send you a copy of the questionnaires by post so your response options are clear while on the telephone.

If you decide to take part, you will be asked whether you consent to the researcher accessing your medical notes and data. If you provide consent, the researcher will only access your medical notes for information about your stroke.

\section{What will I have to do?}

If you decide to take part, you will be asked to complete a set of questionnaires at a time that is convenient for you. You will be contacted, by telephone, after 6 months to repeat the questionnaires.

\section{What are the possible disadvantages and risk of taking part?}

If you find completing the questionnaires raises issues that are distressing, you may find helpful information and insights on the stroke association website (http://www.stroke.org.uk/). But if you remain upset or concerned please contact the principal investigator, Grace Kelly at 02920870587 or the chief investigator, Professor Reg Morris at 02920870582.

\section{What are the possible benefits of taking part?}

We cannot promise the study will help you but the information we get from the study will help to increase the understanding of post-traumatic growth in the research literature.

\section{What if there is a problem?}

If you have a concern about any aspect of this study, you should ask to speak to the researchers who will do their best to answer your questions (contact Grace Kelly on 02920870582 or at kellyg7@cardiff.ac.uk).

If you remain unhappy and wish to complain formally you can do this through Cardiff University on 029 20879131 or at resgov@cardiff.ac.uk 


\section{Will my taking part in the study be kept confidential?}

All information which is collected about you during the course of the research will be kept strictly confidential, and any information about you which leaves the university will have your name and personal details removed so that you cannot be recognised.

Your participation in this research will not impact on the care you receive from other health professionals.

\section{What will happen if I don't carry on with the study?}

If you withdraw from the study all the identifiable information and data collected from you, to date, will be destroyed and your name removed from all study files.

\section{What will happen to the results of the research study?}

It is hoped that the results of the research will be published in a scientific journal. You will be given the opportunity to receive a summary of the findings after the research is complete. You will not be identified in any report/publication related to this research.

\section{Who is organising and funding the research?}

Cardiff University is sponsoring this research.

\section{Who has reviewed the study?}

All research in the NHS is looked at by an independent group of people, called a Research Ethics Committee, to protect your interests. This study has been reviewed and given favourable opinion by the South East Wales Research Ethics Committee.

\section{Further information and contact details.}

For further information about this study, please contact Grace Kelly (Researcher) on 02920870582 or at kellyg7@cardiff.ac.uk 
Appendix F Consent form (version 3)

Version 3

March 2014

\section{Consent Form}

Research Title: Predictors of post-traumatic growth in post-stroke survivors

Name of Researcher: Grace Kelly

Participant identification number: .....

1. I confirm that I have read and understand the information sheet dated March 2014 (version 4) for the above study. I have had the opportunity to consider the information, ask questions and have had these answered satisfactorily.

2. I understand that my participation is voluntary and that I am free to withdraw at any time without giving any reason, without my medical care or legal rights being affected.

3. I understand that relevant sections of my medical notes and data collected during the study will be looked at by the researcher and may be looked at by regulatory authorities or from the NHS Trust or from Cardiff University, where it is relevant to my taking part in this research. I give permission for these individuals to have access to my records.

4. I agree to take part in the above study.

Signature of Participant

Date

Name (Please Print)

Signature of Researcher.

Date

Name (Please Print). 
OPTIONAL:

I would like a summary of the findings of this study sent to my email or postal address below:

(If you would not like to receive a summary of the findings, please leave this section blank)

Email address:

Or

Postal Address (including post code) 


\section{Consent Form}

Research Title: Predictors of post-traumatic growth in post-stroke survivors

Name of Researcher: Grace Kelly

Participant identification number: .....

5. I confirm that I have read and understand the information sheet dated June 2014 (version 5) for the above study. I have had the opportunity to consider the information, ask questions and have had these answered satisfactorily.

6. I understand that my participation is voluntary and that I am free to withdraw at any time without giving any reason, without my medical care or legal rights being affected.

7. I understand that relevant sections of my medical notes and data collected during the study will be looked at by the researcher and may be looked at by regulatory authorities or from the NHS Trust or from Cardiff University, where it is relevant to my taking part in this research. I give permission for these individuals to have access to my records.

8. I agree to take part in the above study.

Signature of Participant

Date

Name (Please Print).

Signature of Researcher.

Date

Name (Please Print).

\section{OPTIONAL:}

I would like a summary of the findings of this study sent to my email or postal address below: 
(If you would not like to receive a summary of the findings, please leave this section blank)

Email address:

Or

Postal Address (including

post code) 
Part of the research infrastructure for Wales funded by the Nationat Institute for Social Care and Health Research, Weish Government. Yn rhan o seilwaith ymchwil Cymru a ariannir gan y Sefydliad Cenedlathol ar gyfer Ymchwil Gofal Cymdethasol ac lechyd, Llywodraeth Cymru

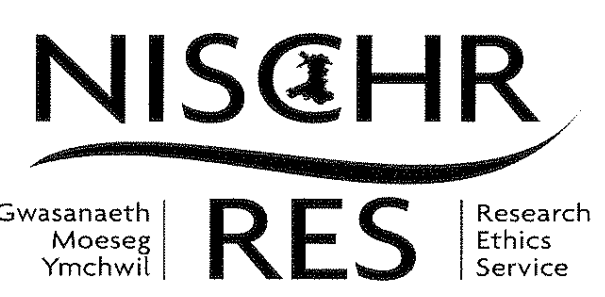

28 March 2014

Professor R Morris

Programme Director

Doctorate Programme in Clinical Psychology

11 th Floor, Tower Building

70 Park Place,

Cathays Park, Cardiff

CF10 3AT

Dear Professor Morris

\section{Study title:}

REC reference:

IRAS project ID:

\section{South East Wales Research Ethics Committee B $6^{\text {th }}$ Floor Churchill House 17 Churchill Way Cardiff CF10 2TW}

Telephone : 02920376823

E-mail : carl.phillips@wales.nhs.uk Website : uwww.nres.nhs.uk

Thank you for your letter of the 5 March 2014, responding to the Committee's request for further information on the above research, and for submitting revised documentation.

The further information has been considered on behalf of the Committee by the Chair. We plan to publish your research summary wording for the above study on the HRA website, together with your contact details, unless you expressly withhold permission to do so.

Publication will be no earlier than three months from the date of this favourable opinion letter. Should you wish to provide a substitute contact point, require further information, or wish to withhold permission to publish, please contact the REC Manager Carl Phillips,

Carl.phillips@wales.nhs.uk.

\section{Confirmation of ethical opinion}

On behalf of the Committee, I am pleased to confirm a favourable ethical opinion for the above research on the basis described in the application form, protocol and supporting documentation [as revised], subject to the conditions specified below.

\section{Ethical review of research sites}

\section{NHS sites}

The favourable opinion applies to all NHS sites taking part in the study, subject to management permission being obtained from the NHS/HSC R\&D office prior to the start of the study (see "Conditions of the favourable opinion" below). 


\section{Conditions of the favourable opinion}

The favourable opinion is subject to the following conditions being met prior to the start of the study.

- Management permission or approval must be obtained from each host organisation prior to the start of the study at the site concerned.

- Management permission ("R\&D approval") should be sought from all NHS organisations involved in the study in accordance with NHS research governance arrangements.

- Guidance on applying for NHS permission for research is available in the Integrated Research Application System or at http://www.rdforum.nhs.uk.

- Where a NHS organisation's role in the study is limited to identifying and referring potential participants to research sites ("participant identification centre"), guidance should be sought from the R\&D office on the information it requires to give permission for this activity.

- For non-NHS sites, site management permission should be obtained in accordance with the procedures of the relevant host organisation.

- Sponsors are not required to notify the Committee of approvals from host organisations

\section{Registration of Clinical Trials}

- All clinical trials (defined as the first four categories on the IRAS filter page) must be registered on a publically accessible database within 6 weeks of recruitment of the first participant (for medical device studies, within the timeline determined by the current registration and publication trees).

- There is no requirement to separately notify the REC but you should do so at the earliest opportunity e.g when submitting an amendment. We will audit the registration details as part of the annual progress reporting process.

- To ensure transparency in research, we strongly recommend that all research is registered but for non clinical trials this is not currently mandatory.

- If a sponsor wishes to contest the need for registration they should contact Catherine Blewett (catherineblewett@nhs.net), the HRA does not, however, expect exceptions to be made. Guidance on where to register is provided within IRAS.

It is the responsibility of the sponsor to ensure that all the conditions are complied with before the start of the study or its initiation at a particular site (as applicable).

\section{Approved documents}

The final list of documents reviewed and approved by the Committee is as follows:

\begin{tabular}{|l|l|l|}
\hline Document & Version & Date \\
\hline Covering Letter & G Kelly & 13 March 2014 \\
\hline Evidence of insurance or indemnity & $\begin{array}{l}\text { UMAL/Cardiff } \\
\text { University }\end{array}$ & 26 July 2013 \\
\hline Investigator CV & G Kelly & 22 January 2014 \\
\hline
\end{tabular}




\begin{tabular}{|l|l|l|}
\hline Investigator CV & R Morris & 01 March 2013 \\
\hline Letter from Sponsor & Cardiff University & 12 December 2013 \\
\hline Letter of invitation to participant & 2 & 01 February 2014 \\
\hline Other: Debrief & 2 & 01 November 2013 \\
\hline Other: A6-1 Revised Summary of Study & 2 & 01 February 2014 \\
\hline Participant Consent Form & 3 & 01 March 2014 \\
\hline Participant Information Sheet & 4 & 01 March 2014 \\
\hline Protocol & 3 & 01 March 2014 \\
\hline Questionnaire: Stroke Survivors' Survey & 2 & 01 December 2013 \\
\hline REC application & 3.5 & 22 January 2014 \\
\hline Response to Request for Further Information & R Morris/G Kelly & 05 March 2014 \\
\hline
\end{tabular}

\section{Statement of compliance}

The Committee is constituted in accordance with the Governance Arrangements for Research Ethics Committees and complies fully with the Standard Operating Procedures for Research Ethics Committees in the UK.

\section{After ethical review}

\section{Reporting requirements}

The attached document "After ethical review - guidance for researchers" gives detailed guidance on reporting requirements for studies with a favourable opinion, including:

- Notifying substantial amendments

- Adding new sites and investigators

- Notification of serious breaches of the protocol

- Progress and safety reports

- Notifying the end of the study

The NRES website also provides guidance on these topics, which is updated in the light of changes in reporting requirements or procedures.

\section{Feedback}

You are invited to give your view of the service that you have received from the National Research Ethics Service and the application procedure. If you wish to make your views known please use the feedback form available on the website.

Further information is available at National Research Ethics Service website > After Review

\section{4/WA/0038 Please quote this number on all correspondence}

We are pleased to welcome researchers and R \& D staff at our NRES committee members' training days - see details at http://www. hra.nhs. uk/hra-training/

With the Committee's best wishes for the success of this project. 


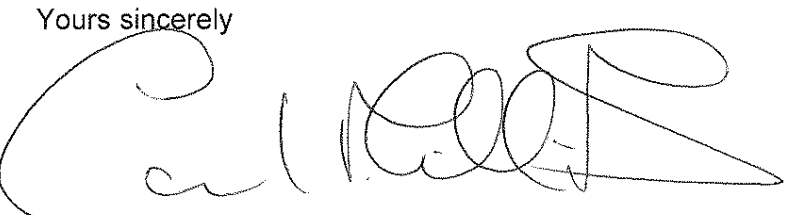

Dr I Doull

Chair, South East Wales Research Ethics Committee B

Enclosures: "After ethical review - guidance for researchers" [SL-AR2]

Copied:- $\quad$ Ms G Kelly, kellyg7@cardiff.ac.uk

Prof R Morris, reg.morris@wales.nhs.uk

R\&D Office for Cardiff University, resgov@cardiff.ac.uk

R\&D Office for Cardiff \& Vale UHB,

CAV research.development@wales.nhs.uk 


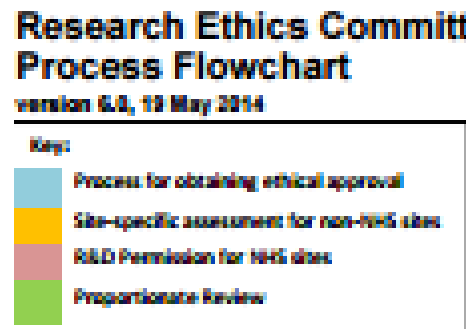

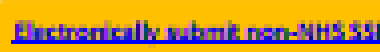
form to HEL for resinew wher KiL form has bann wabminted and viltatsd

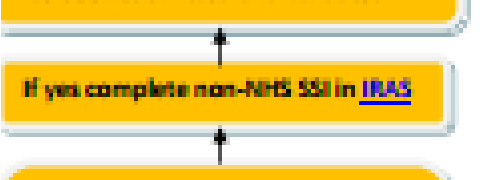

Ior no=-NHE silen antr: Dons your

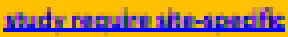

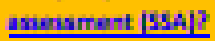

- Baquinamints ber site-ipictic asurisment:

- Luidance on KA Exmption

If you esnuder your study prenints ins mutarial rethal hesea, it min be elintikn for Presortienute Barisw Surate

If Brapartionate Resiru duchien is "Wo Dpiniant. Applataion ruferred ise tull enrmitten
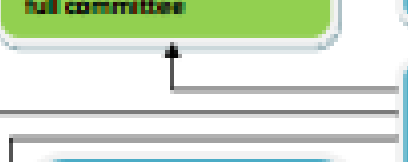

Frosivisnal pirisen pending cons-lation with ipsdalat rithre flosk dors nat ifap

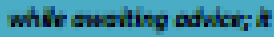
only itop whess adikt sonistred and furthir

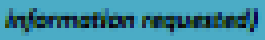

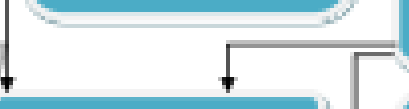

laspunbh Opi-isn with tonditions

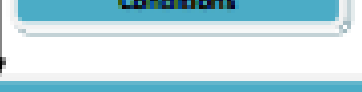

Tavounath Opirim

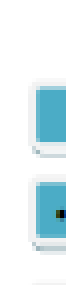

(REC) Application

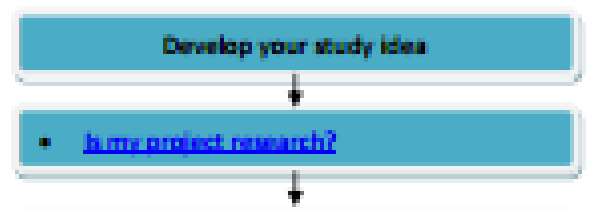

Health Research Authority

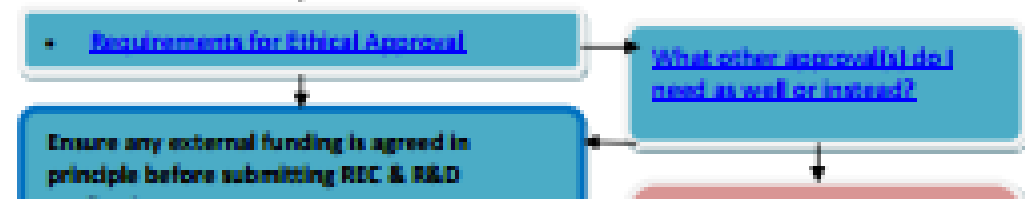
applicatiom

$+$

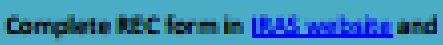

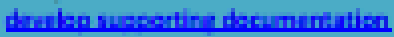

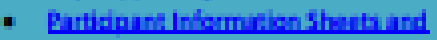
Enuatilers:

- other sarpluindscurnints

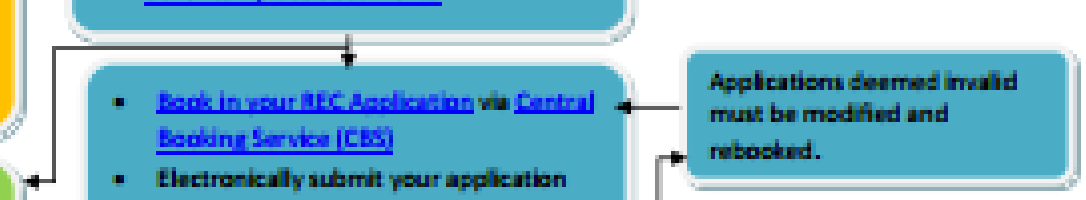

- Ehetrarikaly aubmit pour appleatian and wpporting docu-antation from lises to the ate

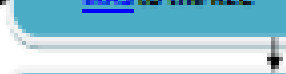

The AEC Manuger vilidaten the applisatien within 5 wring dap of ritipt.

- Vulidaton wil be renfirned by lettir.

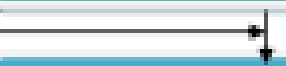

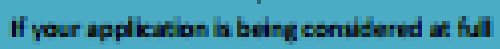

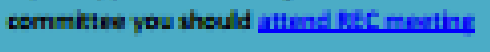

$\neq$

Ipplication risinund bi hEc

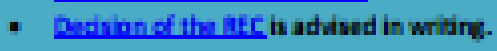

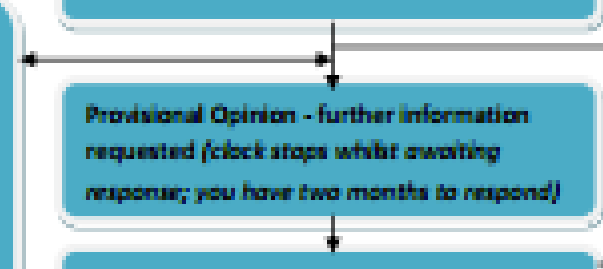

Luknt further information for revire at

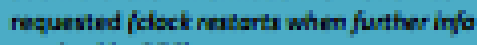
machord by atc

If yeur mipente to Ric is ince-plite, you

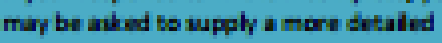

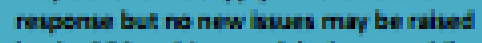

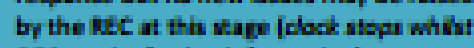

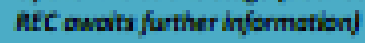

Al remarth inoluhe his menteh ahin requitis Bermbian for ravauth for ach vith

- Compluta upplisationa in $\underline{1045}$ must be medfifed and retaskid.

If your applation is detime rat wall but sutwanding infornation appian rilutivity straqutfor ward, the ALC Manuger miy sentuct pou ind requat: sutstanaine information before it cin be waldatid.

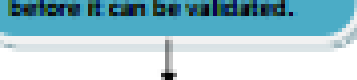

Electrarkaly subrrit

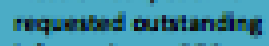
information to Ret

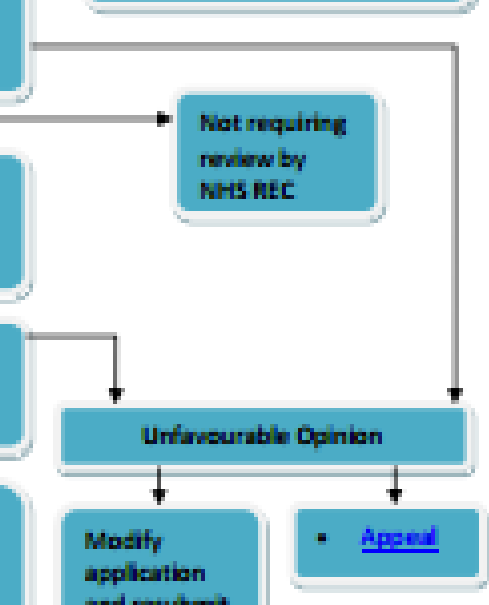




\section{Appendix J Sponsorship letter}

Research, Innovation and Enterprise Services

\author{
12 December 2013 \\ Professor Reg Morris \\ School of Psychology \\ Cardiff University \\ Tower Building \\ 70 Park Place \\ Cardiff \\ CF10 3AT
}

Dear Professor Morris,
Cardiff University

7 th Floo

$30-36$ Newport Road

Cardiff CF24 ODE

Wales UK

Tel Ffôn $+44(0) 2920875834$

Fax Ffacs $+44(0) 2920874189$

Prifysgol Caerdydd

Llawr 7

30 - 36 Newport Road

Caerdydd CF24 ODE

Cymru Y Deyrnas Unedig

Title: Predictors of post-traumatic growth in a post-stroke survivors

I understand that you are acting as Chief Investigator for the above DClinPsy project to be conducted by Grace Kelly

I confirm that Cardiff University agrees in principle to act as Sponsor for the above project, as required by the Research Governance Framework for Health and Social Care.

\section{Scientific Review}

I can also confirm that Scientific Review has been obtained from DClinPsy course tutors.

\section{Insurance}

The necessary insurance provisions will be in place prior to the project commencement. Cardiff University is insured with UMAL. Copies of the insurance certificate are attached to this letter.

\section{Approvals}

On completion of your IRAS form (for NHS REC and NHS R\&D approvals), you will be required to obtain signature from the Sponsor ('Declaration by the Sponsor Representative').

Please then submit the project to the following organisations for approval:

- the appropriate Research Ethics Committee(s);

- National Institute for Social Care Health Research Permissions Coordinating Unit (NISCHR PCU-

\section{Roles and Responsibilities}

As Chief Investigator you have signed a Declaration with the Sponsor to confirm that you will adhere to the standard responsibilities as set out by the Research Governance Framework for Health and Social Care. In accordance with the University's Research Governance Framework, the Chief Investigator is also responsible for ensuring that each research team member is qualified and experienced to fulfill his/her delegated roles including ensuring adequate supervision, support and training.

Roles and responsibilities are adequately detailed in the research protocol.

May I take this opportunity to remind you that, as Chief Investigator, you are required to:

- ensure you are familiar with your responsibilities under the Research Governance Framework for Health and Social Care;

- undertake the study in accordance with Cardiff University's Research Governance Framework and the principles of Good Clinical Practice;

- ensure the Research complies with the Data Protection Act 1998;

- inform Research, Innovation \& Enterprise Services of any amendments to the protocol or study design, including changes to start /end dates;

- co-operate with any audit inspection of the project files or any requests from Research, Innovation $\&$ Enterprise Services for further information. 
Research, Innovation and Enterprise Services

Gwasanaethau Ymchwil, Arloesi a Menter

You should quote the following unique reference number in any correspondence relating to sponsorship for the above project:

$$
\text { SPON 1270-13 }
$$

Cardiff University

This reference number should be quoted on all documentation associated with this project.

Yours sincerely

$$
\text { Whunine. }
$$

Dr K J Pittard Davies

Head of Research Governance and Contracts

Direct line: +44 (0) 2920879274

Email: resgov@cardiff.ac.uk

Cc Grace Kelly

$$
\text { 7th Floor }
$$

$30-36$ Newport Road Cardiff CF24 ODE Wales UK

Tel Ffôn + +44(0)2920875834 Fax Ffacs +44(0)29 20874189 Prifysgol Caerdydd

30 - 36 Newport Road Caerdydd CF24 ODE

Cymru Y Deyrnas Unedig

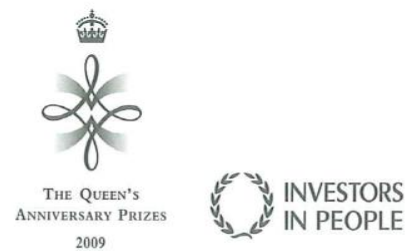


Appendix K R\& D approval letter

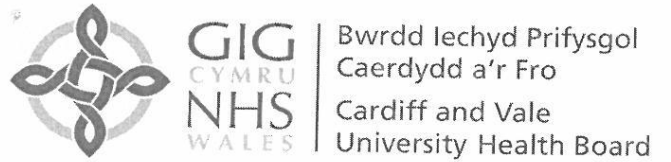

Tel: $\quad 02920746986$

Fax: 02920745311

CAV_Research.Development@wales.nhs.uk
Ysbyty Athrofaol Cymru

University Hospital of Wales

Heath Park.

Cardiff, CF 14 4XW

Phone 0292074774

Parc Y Mynydd Bychan

Caerdydd, CF14 4XW

Fon 0292074774

Minicom 02920743632
From: Professor C Fegan

R\&D Director

R\&D Office, $2^{\text {nd }}$ Floor TB2

University Hospital of Wales

Cardiff

CF14 4XW

10 April 2014

Dr Grace Kelly

Trainee Clinical Psychologist

School of Psychology

Cardiff University

Tower Building

Park Place

Cardiff

CF10 3AT

Dear Dr Kelly

Cardiff and Vale UHB Ref and Study Title : 13/MEH/5815 : Predictors Of PostTraumatic Growth In Post-Stroke Survivors

\section{IRAS Project ID: 140802}

The above project was forwarded to Cardiff and Vale University Health Board R\&D Office by the NISCHR Permissions Coordinating Unit. A Governance Review has now been completed on the project.

Documents approved for use in this study are

\begin{tabular}{|l|l|l|}
\hline Document & Version & Date \\
\hline NHS R\&D Form & 3.5 & Received 10/02/14 \\
\hline SSI Form & 3.5 & Received 10/02/14 \\
\hline Protocol & 3.0 & $01 / 03 / 14$ \\
\hline Participant Information Sheet & 4.0 & $01 / 03 / 14$ \\
\hline Letter of Invitation & 2.0 & $01 / 02 / 14$ \\
\hline Debrief Sheet & 2.0 & $01 / 11 / 13$ \\
\hline Stroke Survivor's Survey & 2.0 & $01 / 12 / 13$ \\
\hline Participant Consent Form & 3.0 & $01 / 03 / 14$ \\
\hline
\end{tabular}


I am pleased to inform you that the UHB has no objection to your proposal and that this study has been classed as pathway-to-portfolio. You have informed us that Cardiff University is willing to act as Sponsor under the Research Governance Framework for Health and Social Care.

Please accept this letter as confirmation of permission for the project to begin within this UHB.

May I take this opportunity to wish you success with the project and remind you that as Principal Investigator you are required to:

- Inform the R\&D Office if this project has not opened within 12 months of the date of this letter. Failure to do so may invalidate R\&D approval.

- Inform NISCHR PCU and the UHB R\&D Office if any external or additional funding is awarded for this project in the future

- Submit any substantial amendments relating to the study to NISCHR PCU in order that they can be reviewed and approved prior to implementation

- Ensure NISCHR PCU is notified of the study's closure

- Ensure that the study is conducted in accordance with all relevant policies, procedures and legislation

- Provide information on the project to the UHB R\&D Office as requested from time to time, to include participant recruitment figures

Yours sincerely,

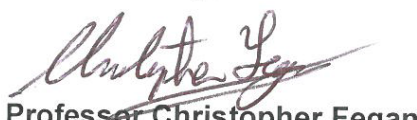

R\&D Director / Chair of the Cardiff and Vale Research Review Service (CaRRS)

CC R\&D Lead Dr Sinead O'Mahoney

Chief Investigator Professor Reg Morris, School for Psychology

Grace Kelly, Trainee Clinical Psychologist, School of Psychology

Chris Shaw, RIES, Cardiff University 


\section{Appendix L Amendment (Summary of changes)}

\section{Summary of changes}

Briefly summarise the main changes proposed in this amendment. Explain the purpose of the changes and their significance for the study.

If this is a modified amendment, please explain how the modifications address the concerns raised previously by the ethics committee.

If the amendment significantly alters the research design or methodology, or could otherwise affect the scientific value of the study, supporting scientific information should be given (or enclosed separately). Indicate whether or not additional scientific critique has been obtained.

Due to difficulties recruiting participants, a substantial amendment is proposed with a revised participant recruitment strategy. Given the limited time-frame of this study (i.e. doctoral research project, resources and prospective design) and the current difficulties in recruiting the target population, it is also proposed that the inclusion criteria are amended from patients two months post-stroke to patients between two to six months post-stroke.

In terms of background, possible participants are currently identified from the stroke register by their stroke clinician and sent a letter of invitation to participate, as well as an information sheet. The letter of invitation outlines that the researcher will be available after the patient's upcoming outpatient appointment if they wish to find out more about the research and/or participate. Participation involves the completion of a battery of questionnaires with the researcher face-to-face (30 minutes) which will be repeated in ten months over the telephone.

Unfortunately, due to organisational factors in how the outpatient clinic is now set up, I have been experiencing difficulty in recruiting participants. I have consulted with the local collaborator, research and development department and the data protection officer for the health board in formulating a proposed substantial amendment.

The proposed amendment to participant recruitment strategy taps into the previously submitted sections of the IRAS form on: summary of study, inclusion criteria, how patients will be invited and, how and by whom potential participants will first be approached. The ways in which the proposed amendment will change the above aspects of design are outlined in the attached protocol (version 4).

In summary, it is proposed that possible participants between two to six months post-stroke are identified from the stroke register and contacted, by telephone, by a known member staff in the stroke team. This staff member will briefly inform the patient of the ongoing research and ask whether they would like to find out more from the researcher. The staff member will explain that the researcher can send an information sheet about the study which would be followed up by a telephone call 4-5 days later to answer any questions that they may have. Patients will be informed that their participation, or not, is entirely voluntary and will in no way impact on their treatment with the team.

If patients are interested in finding out more, they will be asked by the staff member whether they consent to their name, telephone number and address being passed on to the researcher. If they consent, the researcher will post an information sheet to the patient and telephone them 4-5 days later to enquire about whether they have any questions and if they are interested in participating. If patients are interested in participating, the researcher will arrange a time with the patient to either visit them at home or at the University based on what suits the patient best.

Patients who express an interest in participating will be invited to engage in a process of informed consent and provide consent in writing. Patients will be given at least 4-5 days to consider whether they wish to participate. The researcher is a trainee clinical psychologist who has extensive experience of managing the process of informed consent in her routine clinical work. These clinical skills in gaining informed consent are also useful in a research context.

The researcher has the provisional support of the local collaborator and research and development department, subject to the outcome of REC consideration. The researcher has also secured funding, from NISCHR, for the staff member who will be making the phone calls.

In terms of changing the target population from two months post-stroke to between two to six months post-stroke, it is hoped that this will increase the sample size and the potential utility of this study in contributing to the scientific community. The researcher has considered the statistical and scientific validity of this study in light of the proposed amendment and would argue that the proposed amendment will not compromise these aspects of the study. The literature (Tedeschi \& Calhoun, 2004) suggests that people appear to need a period of a few weeks, up to a year or two, to experience some post-traumatic growth. Given that research literature to date has been mainly cross- sectional, it is argued that a prospective study, irrespective of specific time span between stroke and follow-up, will be informative in terms of identifying the predictors of post-traumatic growth over a fixed period of time. 


\section{Any other relevant information}

Applicants may indicate any specific issues relating to the amendment, on which the opinion of a reviewing body is sought.

The researcher has secured funding from NISCHR to pay a staff member from the stroke team to make the telephone calls. The researcher has also consulted with the research and development department at Cardiff and Vale University Health Board who provisionally support the proposed substantial amendment, subject to the decision of the REC committee. The researcher has also consulted with the data protection officer from Cardiff and Vale University Health Board in formulating this proposed substantial amendment. He supports the proposed change in participant recruitment strategy. The researcher plans to adhere to the local health board's lone working policy. 


\section{Appendix M Amendment approval letter}

Part of the research infrastructure for Wales funded by the National institute for Social Care and Heatth Research, Welsh Government. Yy rban o seil waith ymchwil Cymru a ariamir gan y Sefydliad Cenedlaethol ar gyfer Ymchwil Gofal Cymdeithasol ac lechyd, Lilywodraeth Cymru

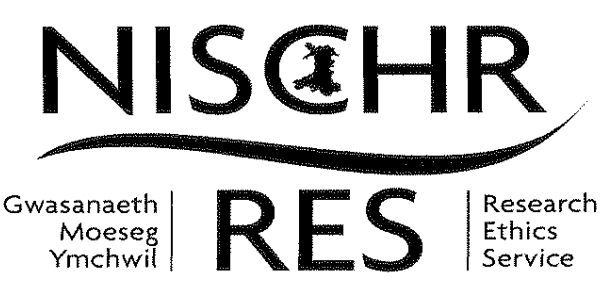

20 June 2014

Prof Reg Morris

Programme Director

Doctorate Programme in Clinical Psychology

Cardiff and Vale University Local Health Board

Doctoral programme in clinical psychology.

11th floor, Tower building

70 Park Place, Cardiff

CF10 3AT

Dear Prof Morris

Study title:

REC reference:

Amendment number:

Amendment date:

IRAS project ID:

Predictors of post-traumatic growth in post-stroke survivors 14/WA/0038

4

01 June 2014

140802

The above amendment was reviewed at the meeting of the Sub-Committee held on the 18 June 2014

\section{Ethical Opinion}

The members of the Committee taking part in the review gave a favourable ethical opinion of the amendment on the basis described in the notice of amendment form and supporting documentation.

\section{Approved documents}

The documents reviewed and approved at the meeting were:

\begin{tabular}{|c|c|c|}
\hline Document: & Version & Date YYYYY \\
\hline Notice of Substantial Amendment (non-CTIMP) & 4 & 01 June 2014 \\
\hline Participant consent form & 4 & 01 June 2014 \\
\hline Participant information sheet (PIS) & 5 & 01 June 2014 \\
\hline Research protocol or project proposal & 1 & 03 June 2014 \\
\hline
\end{tabular}

\section{Membership of the Committee}

The members of the Committee who took part in the review are listed on the attached sheet. 


\section{R\&D Approval}

All investigators and research collaborators in the NHS should notify the R\&D office for the relevant NHS care organisation of this amendment and check whether it affects R\&D approval of the research.

\section{Statement of compliance}

The Committee is constituted in accordance with the Governance Arrangements for Research Ethics Committees and complies fully with the Standard Operating Procedures for Research Ethics Committees in the UK.

We are pleased to welcome researchers and R \& D staff at our NRES committee members' training days - see details at http://www.hra.nhs. uk/hra-training/

\section{4/WA/0038: $\quad$ Please quote this number on all correspondence}

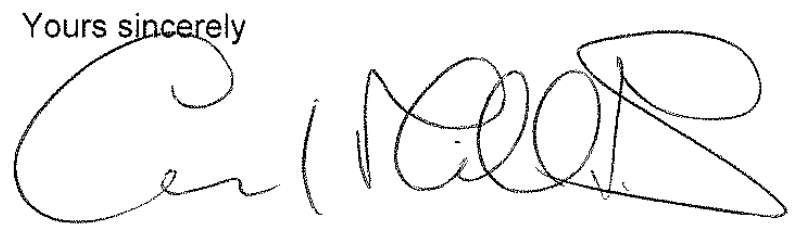

Dr I Doul!

Chair, Wales Research Ethics Committee 2

E-mail: carl.phillips@wales.nhs.uk

Enclosures: List of names and professions of members who took part in the review

Copied:- $\quad$ Ms G Kelly, kellyg7@cardiff.ac.uk

Prof R Morris, reg.morris@wales.nhs.uk

R\&D Office for Cardiff University, resgov@cardiff.ac.uk

R\&D Office for Cardiff \& Vale UHB,

CAV research.development@wales.nhs.uk 
Appendix N Lone worker policy

Available to view online:

http://www.cardiffandvaleuhb.wales.nhs.uk/sitesplus/documents/1143/Lone\% 20Worker\%20Policy\%20v2.reviewpdf.pdf 
Appendix O Stroke survivor survey

\section{Stroke Survivors' Survey}

Instructions:

This questionnaire should take no longer than 30 minutes to complete.

Questionnaires will be anonymous. Therefore, please do not write your name on the questionnaire.

Please try to answer all the questions even if you are unsure about some of them. However, it is your right to stop completing the questionnaire or leave out certain questions at any time should you wish to. 


\section{Part 1: About you ...}

1. Please give your age: years old

2. Please indicate your gender (Please tick the box which applies to you)

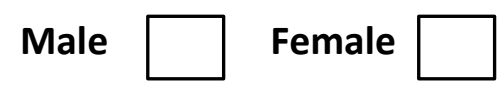

3. Please indicate your ethnicity (Please tick which ever box/boxes applies to you)

British

Irish

Other White

White and Black Caribbean

White and Black African

White and Asian

Any other mixed

Indian

Pakistani

Bangladeshi

Any other Asian
Caribbean

African

Any other Black

Chinese

Other ethnic group
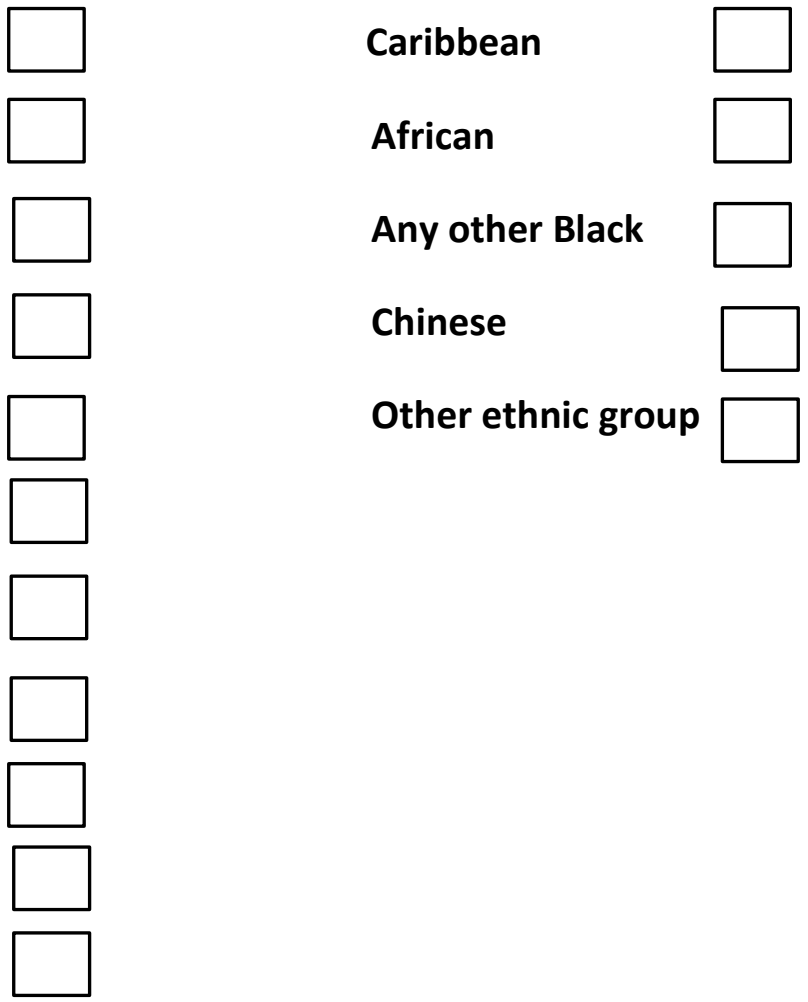

4. Please indicate your occupation (Please tick the box which applies to you)
Retired
Please state your previous job title
In employment
Please state your current job title
Unemployed
If relevant, please state previous job title 
5. When did you have a stroke? (date)

6. Was this the first time you experienced a stroke? (Please tick the box which applies to you)

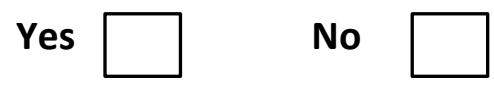

7. What type of stroke did you have? (Please tick the box which applies to you)

Unsure

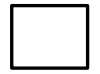

Ischaemic stroke

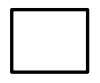

Haemorrhagic stroke

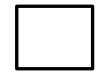

Transient ischaemic attack

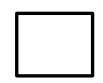

8. Did you have a treatment called thrombolysis? (Please tick the box which applies to you)

$\begin{array}{ll}\text { Unsure } & \square \\ \text { Yes } & \square \\ \text { No } & \square\end{array}$

\section{Since you experienced the stroke ...}

1. Has your ability to communicate with others been affected? (Please tick the box which applies to you)

Not at all

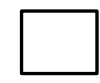

Mildly

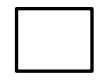

Severe 
2. Do you experience memory difficulties? (Please tick the box which applies to you)

Not at all
Mildly
Severely

3. Have your relationships with those living with you, or those closest to you, changed? (Please tick the box which applies to you)

Not at all

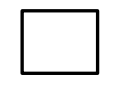

Somewhat

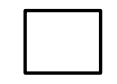

Most definitely

4. Are you currently feeling ... (Please tick the box which applies to you for both feelings categories)

Low in mood or depressed

Anxious or worried

Never

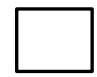

Never

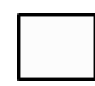

Sometimes

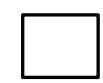

\section{Sometimes}

Often

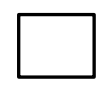

Other

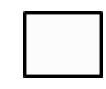

5. In the past two years, have you been treated for depression or anxiety? (Please tick the box which applies to you)

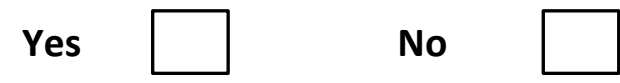

6. Has your sleep been affected? (Please tick the box which applies to you)

Not at all

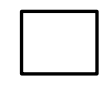

Somewhat

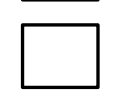

Most definitely 


\section{PART 2: Specific questions about life since the stroke.}

\section{A: COPE (Carver, 1989)}

These items look at the ways you have been coping with the stress in your life since you experienced a stroke. Each item says something about a particular way of coping. I want to know to what extent you've been doing what the item says.

Please do not answer on the basis of whether your efforts to cope are working or not. For the purposes of this survey, I am only interested in what you are doing to cope.

Please rate each item by placing a tick (i.e. $\checkmark$ ) in the 1, 2, 3 or 4 box.

\begin{tabular}{|l|l|l|l|l|}
\hline & $\begin{array}{l}\text { I haven't } \\
\text { been } \\
\text { doing this } \\
\text { at all. }\end{array}$ & $\begin{array}{l}\text { I've been } \\
\text { doing this } \\
\text { a little bit. }\end{array}$ & $\begin{array}{l}\text { I've been } \\
\text { doing this } \\
\text { a medium } \\
\text { amount. }\end{array}$ & $\begin{array}{l}\text { I've been } \\
\text { doing this } \\
\text { a lot. }\end{array}$ \\
\hline $\begin{array}{l}\text { I concentrate my efforts on doing } \\
\text { something about it. }\end{array}$ & & & $\mathbf{2}$ & $\mathbf{4}$ \\
\hline I say to myself "this isn't real". & & & \\
\hline I get used to the idea that it happened. & & & & \\
\hline $\begin{array}{l}\text { I accept that this has happened and that it } \\
\text { can't be changed. }\end{array}$ & & & & \\
\hline $\begin{array}{l}\text { I take additional action to try to get rid of } \\
\text { the problem. }\end{array}$ & & & & \\
\hline I refuse to believe that it has happened. & & & & \\
\hline I pretend that it hasn't really happened. & & & & \\
\hline $\begin{array}{l}\text { I accept the reality of the fact that it } \\
\text { happened. }\end{array}$ & & & & \\
\hline $\begin{array}{l}\text { I take direct action to get around the } \\
\text { problem. }\end{array}$ & & & & \\
\hline I learn to live with it. & & & & \\
\hline I act as though it hasn't even happened. & & & & \\
\hline
\end{tabular}




\section{B: Barthel ADL Index (Collins et al. 1988)}

Sometimes having a stroke can impact how we perform everyday activities. The following questions ask about how you have been coping with everyday tasks over the last two weeks. Please tick the box (i.e. $\checkmark$ ) with the number that describes how independently you are able to do the following the tasks:

\begin{tabular}{|c|c|c|c|c|}
\hline & 0 & 1 & 2 & 3 \\
\hline \multirow[t]{2}{*}{ Feeding } & & & & \\
\hline & Unable & Needs Help & Independent & \\
\hline \multirow[t]{2}{*}{ Bathing/Showering } & & & & \\
\hline & Dependent & Independent & & \\
\hline \multirow[t]{2}{*}{ Grooming } & & & & \\
\hline & Needs help & Independent & & \\
\hline \multirow[t]{2}{*}{ Dressing } & & & & \\
\hline & Dependent & Needs help & Independent & \\
\hline \multirow[t]{2}{*}{ Bowels } & & & & \\
\hline & Incontinent & $\begin{array}{l}\text { Occasional } \\
\text { accident }\end{array}$ & Continent & \\
\hline \multirow[t]{2}{*}{ Bladder } & & & & \\
\hline & Incontinent & $\begin{array}{l}\text { Occasional } \\
\text { accident }\end{array}$ & Continent & \\
\hline \multirow[t]{2}{*}{ Toilet use } & & & & \\
\hline & Dependent & $\begin{array}{l}\text { Needs some } \\
\text { help }\end{array}$ & Independent & \\
\hline \multirow[t]{2}{*}{ Transfers } & & & & \\
\hline & Unable & Major help & Minor help & Independent \\
\hline \multirow[t]{2}{*}{ Mobility } & & & & \\
\hline & Immobile & $\begin{array}{l}\text { Wheelchair } \\
\text { independent }\end{array}$ & $\begin{array}{l}\text { Walks with } \\
\text { help }\end{array}$ & Independent \\
\hline \multirow[t]{2}{*}{ Stairs } & & & & \\
\hline & Unable & Needs help & Independent & \\
\hline
\end{tabular}




\section{C: Rumination Inventory (Calhoun et al. 2000)}

A stroke can be a stressful and traumatic experience/event. Keep this is mind when answering the following questions. Please tick (i.e. $\checkmark$ ) with the box which best describes your experience.

\begin{tabular}{|c|c|c|c|c|}
\hline & Not at all & Rarely & Sometimes & Often \\
\hline $\begin{array}{l}\text { Soon after the event, I thought about the event when I } \\
\text { didn't mean to. }\end{array}$ & & & & \\
\hline $\begin{array}{l}\text { Recently, I have thought about my experience when I } \\
\text { didn't mean to. }\end{array}$ & & & & \\
\hline $\begin{array}{l}\text { Soon after the event, thoughts about the experience } \\
\text { came into my mind and I could not get rid of them. }\end{array}$ & & & & \\
\hline $\begin{array}{l}\text { Recently, thoughts about the event came to my mind } \\
\text { and I could not get rid of them. }\end{array}$ & & & & \\
\hline $\begin{array}{l}\text { Soon after the event, I decided to think about the } \\
\text { experience to try and make sense out of what } \\
\text { happened. }\end{array}$ & & & & \\
\hline $\begin{array}{l}\text { Recently, I decided to think about the experience to try } \\
\text { and make sense out of what happened. }\end{array}$ & & & & \\
\hline $\begin{array}{l}\text { Soon after the event, I tried to make something good } \\
\text { come out of my struggle. }\end{array}$ & & & & \\
\hline $\begin{array}{l}\text { Recently, I have tried to make something good come } \\
\text { out of my struggle. }\end{array}$ & & & & \\
\hline $\begin{array}{l}\text { Soon after the event, I reminded myself of some of the } \\
\text { benefits that came from adjusting to the experience. }\end{array}$ & & & & \\
\hline $\begin{array}{l}\text { Recently, I reminded myself of some of the benefits } \\
\text { that came from adjusting to the experience. }\end{array}$ & & & & \\
\hline $\begin{array}{l}\text { As a result of what happened, soon after the event I } \\
\text { found myself automatically thinking about the purpose } \\
\text { of my life. }\end{array}$ & & & & \\
\hline $\begin{array}{l}\text { As a result of what happened, recently I find myself } \\
\text { automatically thinking about the purpose of my life. }\end{array}$ & & & & \\
\hline $\begin{array}{l}\text { As a result of what happened, soon after the event I } \\
\text { deliberately would think about and ask questions about } \\
\text { whether or not life has a meaning or purpose. }\end{array}$ & & & & \\
\hline $\begin{array}{l}\text { As a result of what happened, recently I will } \\
\text { deliberately think about and ask questions about } \\
\text { whether or not life has a meaning or purpose. }\end{array}$ & & & & \\
\hline
\end{tabular}




\section{D: Post-traumatic growth inventory (Tedeschi \& Calhoun, 1996)}

For each statement below, please indicate the degree to which this change has occurred in your life as a result of having a stroke. Please tick (i.e. $\checkmark$ ) the box with the number which best describes your experience.

Please answer all questions as honestly as you can.

\begin{tabular}{|c|c|c|c|c|c|}
\hline 0 & 1 & 2 & 3 & 4 & 5 \\
\hline $\begin{array}{l}\text { I did not } \\
\text { experience } \\
\text { this change } \\
\text { as a result } \\
\text { of having a } \\
\text { stroke. }\end{array}$ & $\begin{array}{l}\text { I } \\
\text { experienced } \\
\text { this change } \\
\text { to a very } \\
\text { small degree } \\
\text { as a result of } \\
\text { having a } \\
\text { stroke. }\end{array}$ & $\begin{array}{l}\text { I } \\
\text { experienced } \\
\text { this change } \\
\text { to a small } \\
\text { degree as a } \\
\text { result of } \\
\text { having a } \\
\text { stroke. }\end{array}$ & $\begin{array}{l}\text { I } \\
\text { experienced } \\
\text { this change } \\
\text { to a } \\
\text { moderate } \\
\text { degree as a } \\
\text { result of } \\
\text { having a } \\
\text { stroke. }\end{array}$ & $\begin{array}{l}\text { I } \\
\text { experienced } \\
\text { this change } \\
\text { to a great } \\
\text { degree as a } \\
\text { result of } \\
\text { having a } \\
\text { stroke. }\end{array}$ & $\begin{array}{l}\text { I experienced } \\
\text { this change } \\
\text { to a very } \\
\text { great degree } \\
\text { as a result of } \\
\text { having a } \\
\text { stroke. }\end{array}$ \\
\hline
\end{tabular}

\begin{tabular}{|c|c|c|c|c|c|c|}
\hline & 0 & 1 & 2 & 3 & 4 & 5 \\
\hline I changed my priorities about what is important in lif & & & & & & \\
\hline I have a greater appreciation for the value of my owr & & & & & & \\
\hline I developed new interests. & & & & & & \\
\hline I have a greater feeling of self-reliance. & & & & & & \\
\hline I have a better understanding of spiritual matters. & & & & & & \\
\hline I more clearly see that I can count on people in times & & & & & & \\
\hline I established a new path for my life. & & & & & & \\
\hline I have a greater sense of closeness with others. & & & & & & \\
\hline I am more willing to express my emotions. & & & & & & \\
\hline I know better that I can handle difficulties. & & & & & & \\
\hline I am able to do better things with my life. & & & & & & \\
\hline I am better able to accept the ways things work out. & & & & & & \\
\hline
\end{tabular}




\begin{tabular}{|c|c|c|c|c|c|c|}
\hline I can better appreciate each day & & & & & & \\
\hline $\begin{array}{l}\text { New opportunities are available } \\
\text { otherwise. }\end{array}$ & & & & & & \\
\hline I have more compassion for oth & & & & & & \\
\hline & $\mathbf{0}$ & 1 & 2 & 3 & 4 & 5 \\
\hline I put more effort into my relatio & & & & & & \\
\hline I am more likely to try to change & & & & & & \\
\hline I have a stronger religious faith. & & & & & & \\
\hline I discovered that I am stronger $\mathrm{t}$ & & & & & & \\
\hline I learned a great deal about hou & & & & & & \\
\hline I better accept needing others. & & & & & & \\
\hline
\end{tabular}




\section{E: Impact of events scale - revised}

Below is a list of difficulties people sometimes have after stressful life events. Please read each item, and then indicate how distressing each difficulty has been for you during the past seven days with respect to your stroke. Please tick the box (i.e. $\checkmark$ ) which best describes your level of distress.

This assessment is not intended to be a diagnosis. If you are concerned about your results in any way, please speak with your general practitioner or stroke clinician.

\begin{tabular}{|c|c|c|c|c|c|}
\hline & $\begin{array}{l}\text { Not at } \\
\text { all }\end{array}$ & $\begin{array}{l}\text { A little } \\
\text { bit }\end{array}$ & Moderately & $\begin{array}{l}\text { Quite a } \\
\text { bit }\end{array}$ & Extremely \\
\hline \multicolumn{6}{|l|}{$\begin{array}{l}\text { Any reminder brought back feelings } \\
\text { about it. }\end{array}$} \\
\hline \multicolumn{6}{|l|}{ I had trouble staying asleep. } \\
\hline \multicolumn{6}{|l|}{$\begin{array}{l}\text { Other things kept making me think } \\
\text { about it. }\end{array}$} \\
\hline \multicolumn{6}{|l|}{ I felt irritable and angry. } \\
\hline \multicolumn{6}{|l|}{$\begin{array}{l}\text { I avoided letting myself get upset when } \\
\text { reminded of it. }\end{array}$} \\
\hline \multicolumn{6}{|l|}{$\begin{array}{l}\text { I thought about it when I didn't mean } \\
\text { to. }\end{array}$} \\
\hline \multicolumn{6}{|l|}{$\begin{array}{l}\text { I felt as if it hadn't happened or wasn't } \\
\text { real. }\end{array}$} \\
\hline \multicolumn{6}{|l|}{ I stayed away from reminders about it. } \\
\hline \multicolumn{6}{|l|}{ Pictures about it popped into my mind. } \\
\hline \multicolumn{6}{|l|}{ I was jumpy and easily startled. } \\
\hline \multicolumn{6}{|l|}{ I tried not to think about it. } \\
\hline \multicolumn{6}{|l|}{$\begin{array}{l}\text { I was aware that I still had a lot of } \\
\text { feelings about it, but I didn't deal with } \\
\text { them. }\end{array}$} \\
\hline & $\begin{array}{l}\text { Not at } \\
\text { all }\end{array}$ & $\begin{array}{l}\text { A little } \\
\text { bit }\end{array}$ & Moderately & $\begin{array}{l}\text { Quite a } \\
\text { bit }\end{array}$ & Extremely \\
\hline My feelings about it were kind of numb. & & & & & \\
\hline
\end{tabular}




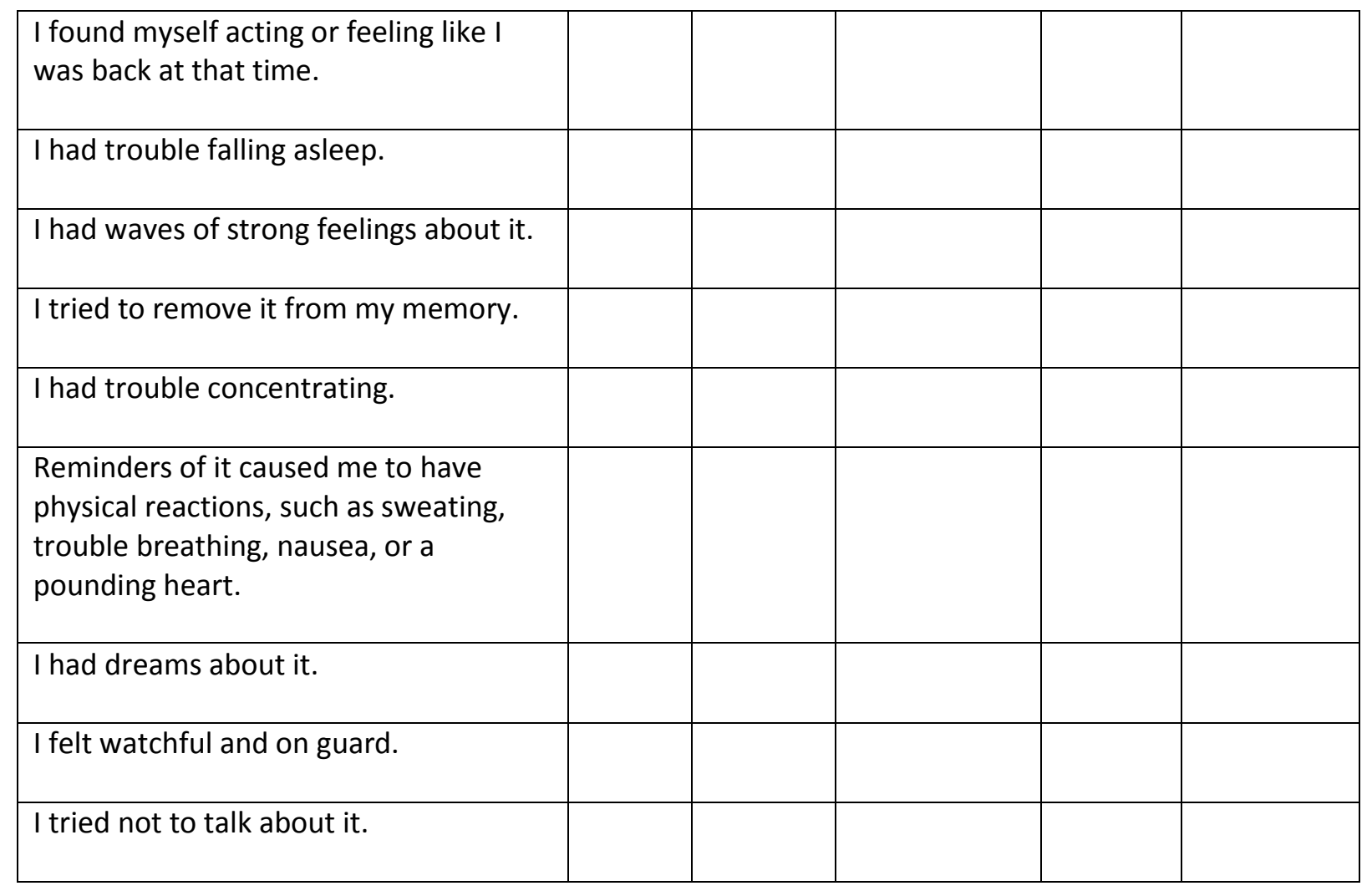




\section{F: Social support - MSPSS (Zimet et al. 1988)}

We are interested in how you feel about the following statements. Read each statement carefully. Indicate how you feel about each statement.

\begin{tabular}{|c|c|c|c|c|c|c|}
\hline 1 & 2 & 3 & 4 & 5 & 6 & 7 \\
\hline $\begin{array}{l}\text { Very } \\
\text { Strongly } \\
\text { Disagree }\end{array}$ & $\begin{array}{l}\text { Strongly } \\
\text { Disagree }\end{array}$ & $\begin{array}{l}\text { Mildly } \\
\text { Disagree }\end{array}$ & Neutral & $\begin{array}{l}\text { Mildly } \\
\text { Agree }\end{array}$ & $\begin{array}{l}\text { Strongly } \\
\text { Agree }\end{array}$ & $\begin{array}{l}\text { Very } \\
\text { Strongly } \\
\text { Agree }\end{array}$ \\
\hline
\end{tabular}

\begin{tabular}{|c|c|c|c|c|c|c|c|}
\hline & 1 & 2 & 3 & 4 & 5 & 6 & 7 \\
\hline There is a special person who is around when I & & & & & & & \\
\hline $\begin{array}{l}\text { There is a special person with whom I can shar } \\
\text { sorrows. }\end{array}$ & & & & & & & \\
\hline My family really tries to help me. & & & & & & & \\
\hline I get the emotional help and support I need fro & & & & & & & \\
\hline I have a special person who is a real source of & & & & & & & \\
\hline My friends really try to help me. & & & & & & & \\
\hline I can count on my friends when things go wron & & & & & & & \\
\hline I can talk about my problems with my family. & & & & & & & \\
\hline I have friends with whom I can share my joys a & & & & & & & \\
\hline There is a special person in my life who cares a & & & & & & & \\
\hline My family is willing to help me make decisions. & & & & & & & \\
\hline I can talk about my problems with my friends. & & & & & & & \\
\hline
\end{tabular}


Appendix P Skewness and kurtosis exceptions to the acceptable range

\begin{tabular}{|l|l|l|}
\hline & Skew & Kurtosis \\
\hline BARTHEL1 & -2.043 & 3.830 \\
\hline IES1 & 5.651 & 3.433 \\
\hline ACTCOP2 & -1.787 & 2.714 \\
\hline ACCEPCOP2 & -2.614 & 8.295 \\
\hline DENCOP2 & 2.766 & 7.603 \\
\hline BARTHEL2 & -2.355 & 4.701 \\
\hline IES2 & & 4.447 \\
\hline MSPSS2 & 2.245 & 1.786 \\
\hline
\end{tabular}


Hi Grace,

I am not an expert in this area but broadly speaking your approach seems reasonable.

I would rely heavily on graphing the results to get a feel that it is all going as you would expect.

Happy to chat if you need more specific advice.

Mark

From: Grace Kelly

Sent: 25 March 2015 12:35

To: Mark Kelson

Cc: reg.morris@wales.nhs.uk

Subject: Statistical Analysis Query

Dear Mark,

I'm one of the students on the DClinPsy programme at the School of Psychology. My thesis supervisor, Prof. Reg Morris, recommended contacting you regarding a query I have about statistical analyses. Several of the variables in my study do not meet the assumptions for parametric methods of analysis such as the pearson product-moment correlation. I am using SPSS software for analysis and noticed an option to 'perform bootstrapping' for bivariate correlations. I have been reading about the use of this analysis in clinical psychology and it seems to have some utility but it appears as though $\mathrm{R}$ software has traditionally been used (Wright et al. 2011).

Given the sample in my study is relatively small and some of the variables are not normally distributed, do you think performing bivariate correlational analysis with bootstrapping using SPSS software would be an appropriate approach to statistical analysis?

Any advice or comments you may have would be much appreciated.

Kind regards,

Grace 
Appendix R Formula used to calculate t-tests

$$
t=\frac{\bar{X}_{1}-\bar{X}_{2}}{\sqrt{\left(\frac{\left(N_{1}-1\right) s_{1}^{2}+\left(N_{2}-1\right) s_{2}^{2}}{N_{1}+N_{2}-2}\right)\left(\frac{1}{N_{1}}+\frac{1}{N_{2}}\right)}}
$$


